\title{
Optical and Radio Properties of Extragalactic Sources Observed by the FIRST Survey and the Sloan Digital Sky Survey
}

\section{Citation}

Ivezić, Željko, Kristen Menou, Gillian R. Knapp, Michael A. Strauss, Robert H. Lupton, Daniel E. Vanden Berk, Gordon T. Richards, et al. 2002. "Optical and Radio Properties of Extragalactic Sources Observed by the FIRST Survey and the Sloan Digital Sky Survey." The Astronomical Journal 124 (5) (November): 2364-2400. doi:10.1086/344069.

\section{Published Version}

doi:10.1086/344069

\section{Permanent link}

http://nrs.harvard.edu/urn-3:HUL.InstRepos:33461895

\section{Terms of Use}

This article was downloaded from Harvard University's DASH repository, and is made available under the terms and conditions applicable to Other Posted Material, as set forth at http:// nrs.harvard.edu/urn-3:HUL.InstRepos:dash.current.terms-of-use\#LAA

\section{Share Your Story}

The Harvard community has made this article openly available.

Please share how this access benefits you. Submit a story.

Accessibility 


\title{
OPTICAL AND RADIO PROPERTIES OF EXTRAGALACTIC SOURCES OBSERVED BY THE FIRST SURVEY AND THE SLOAN DIGITAL SKY SURVEY
}

\author{
Željko Ivezić, ${ }^{1}$ Kristen Menou, ${ }^{1}$ Gillian R. Knapp, ${ }^{1}$ Michael A. Strauss, ${ }^{1}{ }^{1}$ Robert H. Lupton,,${ }^{1}$ Daniel E. Vanden Berk, ${ }^{2}$ \\ Gordon T. Richards, ${ }^{3}$ Christy Tremonti, ${ }^{4}$ Michael A. Weinstein, ${ }^{3}$ Scott Anderson,,${ }^{5}$ Neta A. Bahcall, ${ }^{1}$ \\ Robert H. Becker, ${ }^{6}$ Mariangela Bernardi, ${ }^{7}$ Michael Blanton, ${ }^{8}$ Daniel Eisenstein, ${ }^{9}$ Xiaohui Fan, ${ }^{10}$ \\ Douglas Finkbeiner, ${ }^{1}$ Kristian Finlator,${ }^{1}$ Joshua Frieman, ${ }^{7}$ James E. Gunn, ${ }^{1}$ Pat B. Hall, ${ }^{1,11}$ Rita S. J. Kim, ${ }^{4}$ \\ Ali Kinkhabwala, ${ }^{1}$ Vijay K. Narayanan, ${ }^{1}$ Constance M. Rockosi,${ }^{7}$ David Schlegel, ${ }^{1}$ Donald P. Schneider, ${ }^{3}$ \\ Iskra Strateva, ${ }^{1}$ Mark SubbaRao, ${ }^{7}$ Aniruddha R. Thakar, ${ }^{4}$ Wolfgang Voges, ${ }^{12}$ Richard L. White, ${ }^{13}$ Brian Yanny, ${ }^{2}$ \\ Jonathan Brinkmann, ${ }^{14}$ Mamoru Doi, ${ }^{15}$ Masataka Fukugita, ${ }^{16}$ Gregory S. Hennessy, ${ }^{17}$ Jeffrey A. Munn, ${ }^{18}$ \\ Robert C. Nichol, ${ }^{19}$ and Donald G. York ${ }^{7}$ \\ Received 2002 February 21; accepted 2002 August 7
}

\begin{abstract}
We discuss the optical and radio properties of $\sim 30,000$ FIRST (radio, $20 \mathrm{~cm}$, sensitive to $1 \mathrm{mJy}$ ) sources positionally associated within 1".5 with a Sloan Digital Sky Survey (SDSS) (optical, sensitive to $r^{*} \sim 22.2$ ) source in $1230 \mathrm{deg}^{2}$ of sky. The matched sample represents $\sim 30 \%$ of the 108,000 FIRST sources and $0.1 \%$ of the $2.5 \times 10^{7}$ SDSS sources in the studied region. SDSS spectra are available for 4300 galaxies and 1154 quasars from the matched sample and for a control sample of 140,000 galaxies and 20,000 quasars in 1030 $\mathrm{deg}^{2}$ of sky. Here we analyze only core sources, which dominate the sample; the fraction of SDSS-FIRST sources with complex radio morphology is determined to be less than $10 \%$. This large and unbiased catalog of optical identifications provides much firmer statistical footing for existing results and allows several new findings. The majority ( $83 \%$ ) of the FIRST sources identified with an SDSS source brighter than $r^{*}=21$ are optically resolved; the fraction of resolved objects among the matched sources is a function of the radio flux, increasing from $\sim 50 \%$ at the bright end to $\sim 90 \%$ at the FIRST faint limit. Nearly all optically unresolved radio sources have nonstellar colors indicative of quasars. We estimate an upper limit of $\sim 5 \%$ for the fraction of quasars with broadband optical colors indistinguishable from those of stars. The distribution of quasars in the radio flux-optical flux plane suggests the existence of the "quasar radio dichotomy"; $8 \% \pm 1 \%$ of all quasars with $i^{*}<18.5$ are radio-loud, and this fraction seems independent of redshift and optical luminosity. The radio-loud quasars have a redder median color by $0.08 \pm 0.02 \mathrm{mag}$, and show a 3 times larger fraction of objects with extremely red colors. FIRST galaxies represent 5\% of all SDSS galaxies with $r^{*}<17.5$, and 1\% for $r^{*}<20$, and are dominated by red $\left(u^{*}-r^{*}>2.22\right)$ galaxies, especially those with $r^{*}>17.5$. Magnitudeand redshift-limited samples show that radio galaxies have a different optical luminosity distribution than nonradio galaxies selected by the same criteria; when galaxies are further separated by their colors, this result remains valid for both blue and red galaxies. For a given optical luminosity and redshift, the observed optical colors of radio galaxies are indistinguishable from those of all SDSS galaxies selected by identical criteria. The distributions of radio-to-optical flux ratio are similar for blue and red galaxies in redshift-limited samples; this similarity implies that the difference in their luminosity functions and resulting selection effects are the dominant cause for the preponderance of red radio galaxies in flux-limited samples. The fraction of radio galaxies whose emission-line ratios indicate an AGN (30\%), rather than starburst, origin is 6 times larger than the corresponding fraction for all SDSS galaxies $\left(r^{*}<17.5\right)$. We confirm that the AGN-tostarburst galaxy number ratio increases with radio flux and find that radio emission from AGNs is more concentrated than radio emission from starburst galaxies.
\end{abstract}

Key words: galaxies: active — galaxies: photometry — quasars: general — radio continuum On-line material: color figures

\footnotetext{
${ }_{1}^{1}$ Princeton University Observatory, Peyton Hall, Princeton, NJ 08544-1001.

${ }^{2}$ Fermi National Accelerator Laboratory, P.O. Box 500, Batavia, IL 60510.

${ }^{3}$ Department of Astronomy and Astrophysics, 525 Davey Laboratory, Pennsylvania State University, University Park, PA 16802.

${ }^{4}$ Department of Physics and Astronomy, John Hopkins University, 3400 North Charles Street, Baltimore, MD 21218.

${ }^{5}$ Department of Astronomy, University of Washington, Box 351580, Seattle, WA 98195.

${ }^{6}$ Department of Physics, University of California, Davis, Davis, CA 95616.

${ }^{7}$ Department of Astronomy and Astrophysics, University of Chicago, 5640 South Ellis Avenue, Chicago, IL 60637.

${ }^{8}$ Department of Physics, New York University, 4 Washington Place, New York, NY 10003.

${ }^{9}$ Steward Observatory, University of Arizona, 933 North Cherry Avenue, Tucson, AZ 85721

${ }^{10}$ Institute for Advanced Study, Einstein Drive, Princeton, NJ 08540.

${ }^{11}$ Departamento de Astronomía y Astrofísica, Pontificia Universidad Católica de Chile, Facultad de Física, Casilla 306, Santiago 22, Chile.

12 Max-Planck-Institüt für Extraterrestrische Physik, Postfach 1317, D-85741 Garching, Germany.

${ }^{13}$ Space Telescope Science Institute, 3700 San Martin Drive, Baltimore, MD 21218.

${ }^{14}$ Apache Point Observatory, P.O. Box 59, Sunspot, NM 88349.

15 Institute of Astronomy and Research Center for the Early Universe, University of Tokyo, 2-21-1 Osawa, Mitaka, Tokyo 181-0015, Japan.

${ }^{16}$ Institute for Cosmic Ray Research, University of Tokyo, 5-1-5 Kashiwa, Kashiwa City, Chiba 277-8582, Japan.

${ }_{17}$ US Naval Observatory, 3450 Massachusetts Avenue, NW, Washington, DC 20392-5420.

18 US Naval Observatory, Flagstaff Station, P.O. Box 1149, Flagstaff, AZ 86002.

${ }^{19}$ Department of Physics, Carnegie Mellon University, 5000 Forbes Avenue, Pittsburgh, PA 15232.
} 


\section{INTRODUCTION}

Statistical studies of the radio emission from extragalactic sources are entering a new era as a result of the availability of large sky area, high-resolution radio surveys that are sensitive to millijansky levels (e.g., FIRST, Becker, White, \& Helfand 1995; NVSS, Condon et al. 1998; for an informative overview and a comparison of modern radio surveys see De Breuck 2000). However, to fully utilize the strength of these new radio surveys, the optical properties of the sources must be determined. The Sloan Digital Sky Survey (SDSS, York et al. 2000) is a good match in areal coverage and depth $\left(r^{*} \sim 22.2\right)$ to the new radio surveys. The SDSS is producing five-color optical images and photometry for more than $10^{8}$ Galactic and extragalactic sources, as well as spectra for about $10^{6}$ galaxies and $10^{5}$ quasars. The accurate photometry and detailed morphological and spectroscopic information can be used to efficiently separate sources into stars, quasars and galaxies, and to study in detail the optical properties of radio sources.

In this work we discuss the properties of sources observed by the FIRST and SDSS surveys. We use the FIRST survey because it has superior astrometric accuracy, resolution, and faint sensitivity limit compared with other contemporary large-area radio surveys (e.g., NVSS). We use the SDSS because it has the best photometric and astrometric accuracy available for a large-area optical survey, five-band data extending from 3000 to $10,000 \AA$, and spectra for a large number of extragalactic sources. The largest previous samples of optical identifications for FIRST sources are based on the APM survey. McMahon et al. (2001) describe an identification program for 382,892 FIRST sources from $4150 \mathrm{deg}^{2}$ of the north Galactic cap, which resulted in $\sim 70,000$ optical counterparts, and Magliocchetti \& Maddox (2002a) present a detailed analysis for $\sim 4000$ sources. The sample discussed here, with SDSS identification for $\sim 30,000$ FIRST sources, has the advantage of more than 5 times more accurate optical astrometry and photometry; it is also about 1 mag deeper and utilizes five-color, instead of two-color, optical information. In addition, spectra are available for a subset of 5454 matched sources.

A brief description of the FIRST and SDSS data is provided in $\S 2$. We describe the matched data and the basic matching statistics in $\S 3$. A more detailed study of the optical and radio properties of quasars and galaxies is presented in $\S 4$ and $\S 5$, and we discuss our results in $\S 6$.

\section{THE SURVEYS}

\subsection{Sloan Digital Sky Survey}

\subsubsection{Technical Summary}

The $\operatorname{SDSS}^{20}$ is a digital photometric and spectroscopic survey, which will cover one-quarter of the celestial sphere in the north Galactic cap and produce a smaller area $(\sim 225$ $\mathrm{deg}^{2}$ ) but much deeper survey in the southern Galactic hemisphere (York et al. 2000; Stoughton et al. 2002, hereafter EDR, and references therein). The flux densities of detected objects are measured almost simultaneously in five bands $(u, g, r, i$, and $z$; Fukugita et al. 1996) with effective wavelengths of 3551, 4686, 6166, 7480, and $8932 \AA$ (Gunn et al.

${ }^{20}$ See http://www.astro.princeton.edu/PBOOK/welcome.htm.
1998). The resulting catalog is $95 \%$ complete $^{21}$ for point sources to limiting AB magnitudes of 22.0, 22.2, 22.2, 21.3, and 20.5 in the north Galactic $\operatorname{cap}^{22}$ in $u, g, r, i$, and $z$, respectively. The eventual survey sky coverage of about $\pi$ steradians $\left(10,000 \mathrm{deg}^{2}\right)$ will result in photometric measurements to the above detection limits for about 100 million stars. Astrometric positions are accurate to about 0."1 (rms per coordinate) for sources brighter than $20.5 \mathrm{mag}$, and the morphological information from the images allows robust star-galaxy separation down to $r^{*} \sim 21.5 \mathrm{mag}$ (Lupton et al. 2002). The spectra have a resolution of 1800-2000 in the wavelength range from 3800-9200 A. Extragalactic sources targeted in the SDSS spectroscopic survey include a fluxlimited "main" galaxy sample $\left(r^{*}<17.77\right.$, Strauss et al. 2002), the luminous red galaxy sample (Eisenstein et al. 2001, hereafter E01), and quasars (Richards et al. 2002). Further technical details about the SDSS data can be found in EDR and references therein.

\subsubsection{SDSS Color-Color and Color-Magnitude Diagrams}

The position of an object in SDSS color and magnitude space can be used to constrain its nature, thus providing an efficient method to analyze the properties of optically identified radio sources. The color-color and color-magnitude diagrams that summarize the photometric properties of SDSS sources are shown in Figure 1. In this and all other figures we correct the optical magnitudes for interstellar extinction, determined from the maps of Schlegel, Finkbeiner, \& Davis (1998). Typical $r$-band absorption values $\left(A_{r}=0.84 A_{V}\right)$ for the high-latitude regions discussed in this work are 0.05 to 0.15 . Throughout this work we use "model" magnitudes, ${ }^{23}$ as computed by the photometric pipeline (PHOTO, version v5_2; for details see Lupton et al. 2002 and EDR). The model magnitudes are measured by fitting an exponential and a de Vaucouleurs profile of arbitrary inclination to the two-dimensional image and convolved with the local point-spread function, and using the formally better model in the $r$ band to evaluate the magnitude. Photometric errors are typically $0.03 \mathrm{mag}$ at the bright end $\left(r^{*}<20 \mathrm{mag}\right)$ and increase to about $0.1 \mathrm{mag}$ at $r^{*} \sim 21 \mathrm{mag}$, the faint limit relevant in this work (for more details see Ivezić et al. 2000 and Strateva et al. 2001, hereafter S01).

The top left panel in Figure 1 displays the $g^{*}-r^{*}$ versus $u^{*}-g^{*}$ color-color diagram for $\sim 300,000$ objects with errors less than $0.1 \mathrm{mag}$ in the plotted bands, observed in $50 \mathrm{deg}^{2}$ of sky during the SDSS commissioning phase. The unresolved sources are shown by dots, and the resolved sources by linearly spaced contours. The low-redshift quasars $(z \lesssim 2.5)$, selected by their blue $u^{*}-g^{*}$ colors indicating UV excess (hereafter UVX, roughly in the region $\left.-0.6<u^{*}-g^{*}<0.6,-0.2<g^{*}-r^{*}<0.6\right)$, are shown as

${ }^{21}$ These values are determined by comparing multiple scans of the same area obtained during the commissioning year. Typical seeing in these observations was 1 ."6 $6 \pm 0$ ". 2 .

${ }^{22} \mathrm{We}$ refer to the measured magnitudes in this paper as $u^{*}, g^{*}, r^{*}, i^{*}$, and $z^{*}$ because the absolute calibration of the SDSS photometric system is still uncertain at the $\lesssim 0.05$ mag level. The SDSS filters themselves are referred to as $u, g, r, i$, and $z$. All magnitudes are given on the $\mathrm{AB}_{\nu}$ system (Oke \& Gunn 1983); for additional discussion regarding the SDSS photometric system see Fukugita et al. (1996).

${ }^{23}$ Note that the SDSS photometric system uses asinh magnitudes (Lupton, Gunn, \& Szalay 1999). 

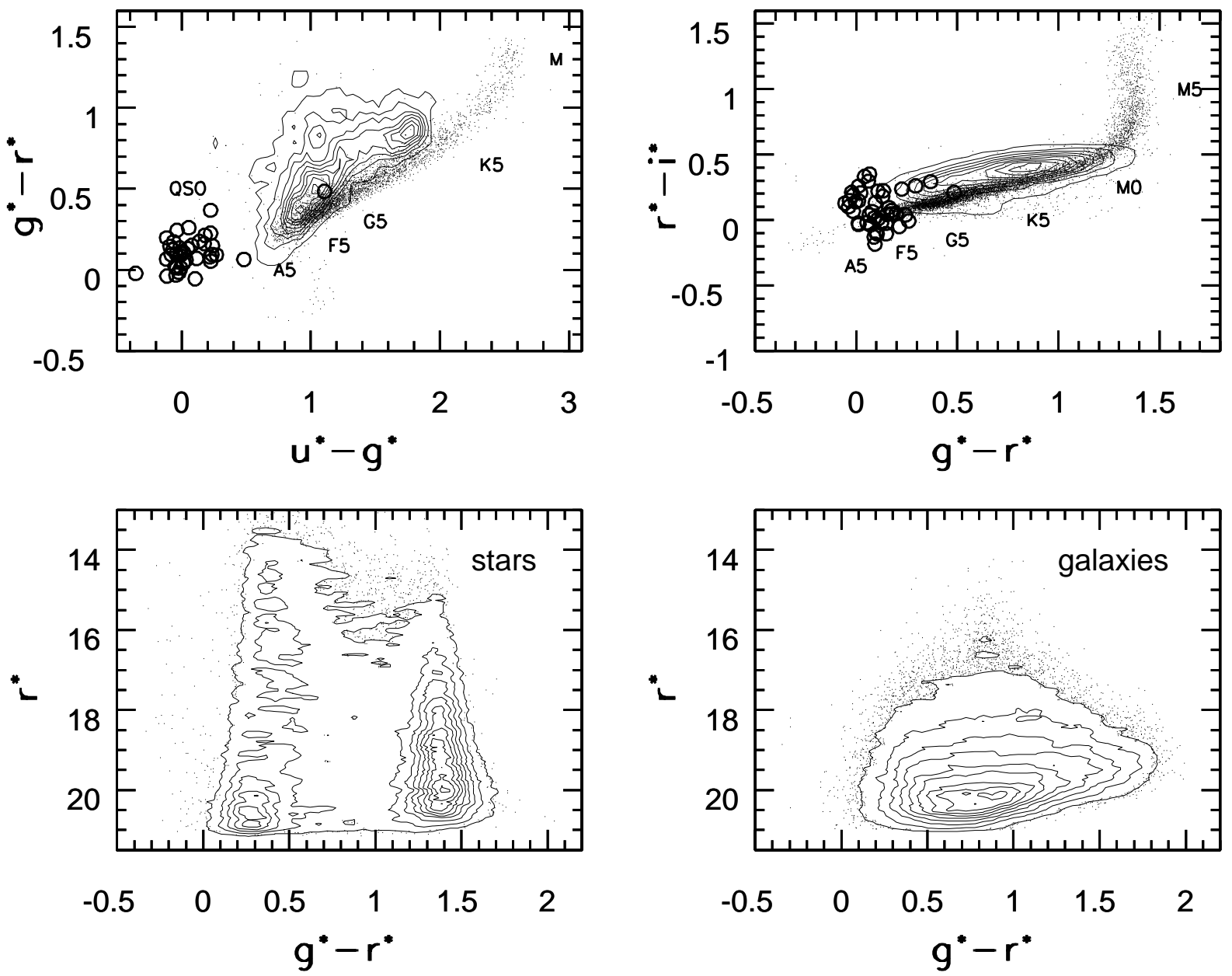

FIg. 1.-SDSS color-color and color-magnitude diagrams for $\sim 300,000$ objects observed in $50 \mathrm{deg}^{2}$ of sky. The top two panels show color-color diagrams for objects with photometric errors less than $0.1 \mathrm{mag}$ in the plotted bands. The unresolved sources are shown as dots, and the distribution of resolved sources is shown by linearly spaced density contours. The low-redshift quasars $(z \lesssim 2.5)$, selected by their blue $u^{*}-g^{*}$ colors, are shown as circles. Almost all of the unresolved sources marked as dots are stars (a small fraction may be quasars and compact galaxies), with approximate spectral types as marked. The bottom two panels show color-magnitude diagrams for unresolved (left) and resolved (right) sources.

circles (for more details about SDSS quasar targeting strategy, see Richards et al. 2002). Most of the unresolved sources are stars. The position of a star in color-color diagrams is mainly determined by its spectral type (Finlator et al. 2000, hereafter F00 and references therein). For most of its length the locus in the $g^{*}-r^{*}$ versus $u^{*}-g^{*}$ diagram consists of stars with spectral types ranging from $\mathrm{F}$ to late $\mathrm{K}$, with late $\mathrm{K}$ and $\mathrm{M}$ stars distributed at the red end of the stellar locus, as marked in the figure (in all color-color diagrams red is toward the upper right). Different $\mathrm{M}$ spectral subtypes cannot be distinguished in the $g^{*}-r^{*}$ versus $u^{*}-g^{*}$ diagram and are better separated in the $r^{*}-i^{*}$ versus $g^{*}-r^{*}$ diagram (Fig. 1, top right), where they occupy the vertical part of the stellar locus with $g^{*}-r^{*} \sim 1.4$. The modeling of the stellar populations observed by SDSS (F00) indicates that the vast majority of these stars (about 99\%) are on the main sequence.

The bottom two panels in Figure 1 display the colormagnitude diagrams for unresolved (left) and resolved $($ right $)$ sources. Blue stars $\left(g^{*}-r^{*}<1\right)$ are more luminous than red stars $\left(g^{*}-r^{*}>1\right)$ and are thus observable to comparatively larger distances (about $1-10 \mathrm{kpc}$ for blue stars vs. $0.1-1 \mathrm{kpc}$ for red stars). This, plus the steeply increasing luminosity function for the less luminous red stars, gives rise to the observed bimodal stellar distribution (for details see Chen et al. 2001). The distribution of the $g^{*}-r^{*}$ color for galaxies is very narrow $(\lesssim 0.2 \mathrm{FWHM})$ for $r^{*} \lesssim 16$ and widens considerably for fainter objects; the width of the $g^{*}-r^{*}$ distribution is significantly larger than the photometric errors ( 1 vs. $<0.05 \mathrm{mag}$ ) and thus represents a real spread in colors. The asymmetric distribution suggests that there are at least two galaxy populations.

The distribution of galaxies in SDSS color-color space has been studied by Shimasaku et al. (2001) and S01. S01 found that galaxies show a strongly bimodal distribution of $u^{*}-r^{*}$ color (also visible in Fig. 1, top left) and demonstrated that the two groups can be associated with late-type (blue group) and early-type (red group) galaxies. However, faint red galaxies (which dominate the radio galaxy sample) have poorly determined $u$-band magnitudes, not allowing reliable galaxy-type separation on the basis of color. Thus, here we separate the two classes in the $r^{*}$ versus $g^{*}-r^{*}$ color-magnitude diagram. Figure 2 shows such diagrams for $\sim 190,000$ galaxies from $\sim 100 \mathrm{deg}^{2}$ of sky, separated according to the $u^{*}-r^{*}$ criterion proposed by S01: the top panel shows $\sim 126,000$ galaxies with $u^{*}-r^{*}<2.22$, and the 

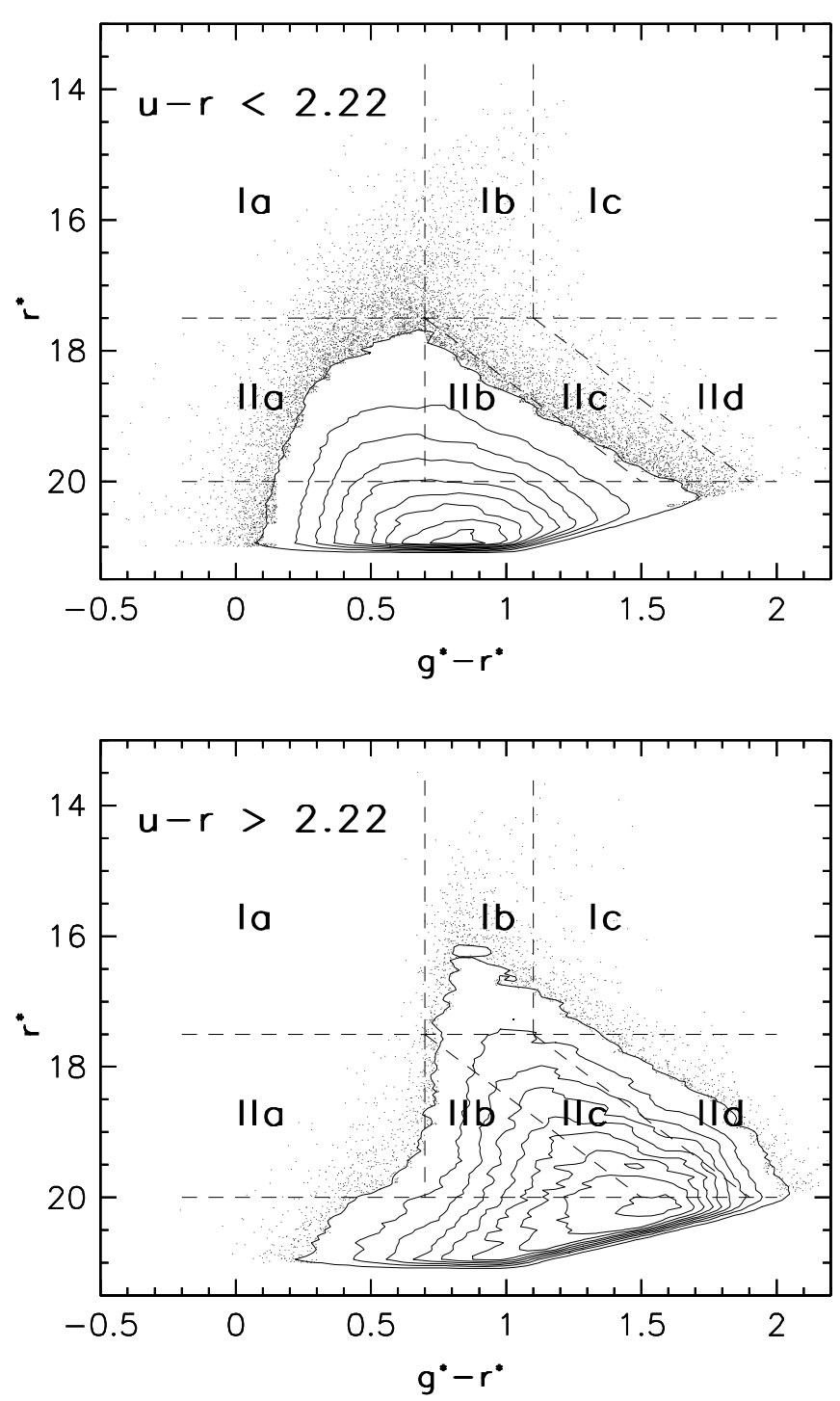

FIG. 2.-The $r^{*}$ vs. $g^{*}-r^{*}$ color-magnitude diagrams for $\sim 190,000$ galaxies separated by their $u^{*}-r^{*}$ color. Top: $\sim 126,000$ blue galaxies with $u^{*}-r^{*}<2.22$. Bottom: $\sim 64,000$ red galaxies with $u^{*}-r^{*}>2.22$. The faint-end distribution shape is due to magnitude cutoffs. The dashed lines outline several characteristic regions, which are useful when analyzing the properties of galaxies (see text for details).

bottom panel shows $\sim 64,000$ galaxies with $u^{*}-r^{*}>2.22$. Figure 2 demonstrates that an approximate separation of the two basic galaxy types is possible even when only the $g^{*}$ and $r^{*}$-band magnitudes are used. The mixing of the two types in the central region (IIb) of the diagram is probably a consequence of the fact that the $u$-band magnitudes become less accurate at the faint end, but $K$-correction effects may also play a role.

The dashed lines in Figure 2 divide the $r^{*}$ versus $g^{*}-r^{*}$ color-magnitude diagram into several characteristic regions, which are a convenient analysis tool when studying various subsamples of galaxies. Galaxies brighter than $r^{*}=17.5$ (close to the limit of $r^{*}=17.77$ for the SDSS main spectroscopic galaxy sample; see Strauss et al. 2002) display a very narrow $g^{*}-r^{*}$ color distribution. We divide this magnitude range into a region including the core of the distribution (Ib) and the two regions bluer (Ia) and redder (Ic) than the core.
The regions with $17.5<r^{*}<20$ are defined by following the boundaries of the distribution of the two galaxy types: the region IIa is dominated by blue galaxies, while the regions IIc and IId are dominated by red galaxies. The IIb region contains substantial fractions of both galaxy types. The distinction between IIc and IId regions is made, somewhat arbitrarily, by extending the separation between the $\mathrm{Ib}$ and Ic regions parallel to the boundary between the IIb and IIc regions. The definition of region IId is practically identical to the SDSS spectroscopic targeting boundary for luminous red galaxies (E01). Table 1 shows the counts of galaxies per unit solid angle $\left(\mathrm{deg}^{-2}\right)$ and the fraction of blue $\left(u^{*}-r^{*}<2.22\right)$ and red $\left(u^{*}-r^{*}>2.22\right)$ galaxies in each region.

\subsection{FIRST Survey}

The Faint Images of the Radio Sky at Twenty cm survey (FIRST, Becker et al. 1995) is using the Very Large Array (VLA) to produce a map of the $20 \mathrm{~cm}(1.4 \mathrm{GHz})$ sky with a beam size of 5".4 and an rms sensitivity of about $0.15 \mathrm{mJy}$ beam ${ }^{-1}{ }^{24}$ The survey will cover an area of about 10,000 $\mathrm{deg}^{2}$ in the north Galactic cap and a smaller area along the celestial equator, corresponding to the sky regions observed by SDSS. With a source surface density of $\sim 90 \mathrm{deg}^{-2}$, the final catalog will include $\sim 10^{6}$ sources. The survey is currently $70 \%$ complete. At the $1 \mathrm{mJy}$ source detection threshold about $35 \%$ of FIRST sources have resolved structure ${ }^{25}$ on scales from $2^{\prime \prime}-30^{\prime \prime}$.

The FIRST catalog lists two types of $20 \mathrm{~cm}$ continuum flux density: the peak value, $F_{\text {peak }}$, and the integrated flux density, $F_{\text {int }}$. These measurements are derived from fitting a two-dimensional Gaussian to each source, where the source maps are generated from the co-added images from 12 pointings. For convenience we define an "AB radio magnitude" as

$$
t=-2.5 \log \left(\frac{F_{\text {int }}}{3631 \mathrm{Jy}}\right),
$$

which places the radio magnitudes on the $\mathrm{AB}_{\nu}$ system of Oke \& Gunn (1983). One of the advantages of that system is that the zero point $(3631 \mathrm{Jy})$ does not depend on wavelength.

To quantify the radio morphology, we define a dimensionless measure of concentration

$$
\theta=\left(\frac{F_{\text {int }}}{F_{\text {peak }}}\right)^{1 / 2}
$$

Sources with resolved radio emission will have $\theta>1$.

The FIRST sensitivity limit of $1 \mathrm{mJy}$ (for the peak flux density) corresponds to $t=16.4$; we used the total flux, $F_{\text {int }}$, to define the $t$ magnitude. The various spectral indices (defined by $F_{\nu} \propto \nu^{\alpha}$ ) that can be formed between an SDSS wavelength with a measured magnitude $m$ and the FIRST wavelength are then

$$
\alpha_{t m}=\frac{0.4}{\log \left(20 \mathrm{~cm} / \lambda_{m}\right)}(t-m),
$$

\footnotetext{
${ }^{24}$ See http://sundog.stsci.edu.

${ }^{25}$ Some FIRST sources are overresolved and have underestimated fluxes (for more details see Becker et al. 1995 and §3.9).
} 
TABLE 1

Galaxy Distribution in the SDSS $r$ * versus $g^{*}-r *$ Color-Magnitude Diagrama

\begin{tabular}{llrrrr}
\hline \hline \multicolumn{1}{c}{ Region } & \multicolumn{1}{c}{ Definition }
\end{tabular}

a See Fig. 22.

${ }^{\mathrm{b}} \mathrm{L} 1=r^{*}-\left[15.30+3.13^{*}\left(g^{*}-r^{*}\right)\right] ; \mathrm{L} 2=r^{*}-\left[14.06+3.13^{*}\left(g^{*}-r^{*}\right)\right]$.

c Galaxies with $u^{*}-r^{*}<2.22$.

d Galaxies with $u^{*}-r^{*}>2.22$

${ }^{e}$ Fraction detected by FIRST.

where $\lambda_{m}$ is the effective wavelength corresponding to $m$. For example, for the $i$ band $\left(\lambda_{i}=7480 \AA\right)$

$$
\alpha_{t i}=0.0737\left(t-i^{*}\right) .
$$

We will also find it useful to define the ratio of the radio to optical flux density (without including the $K$-correction ${ }^{26}$ ) as

$$
R_{m}=\log \left(F_{\text {radio }} / F_{\text {optical }}\right)=0.4(m-t),
$$

where $m$ is one of the SDSS magnitudes. In this work we use $R_{r}, R_{i}$, and $R_{z}$. Note that some papers define $R$ without the logarithm.

\section{THE MATCHED DATA AND THE MATCHING STATISTICS}

Here we present a brief summary of SDSS and FIRST data used in this work. We also describe the matching algorithm, discuss the star-galaxy separation, and analyze the radio differences between matched and unmatched sources and between optically unresolved and resolved matched sources.

\subsection{SDSS Data}

We utilize SDSS imaging and spectroscopic observations that were reduced and calibrated prior to 2001 October 8 and that overlap the area already scanned by the FIRST survey. The imaging data cover a $1230 \mathrm{deg}^{2}$ large area on the sky and include $2.53 \times 10^{7}$ unique unsaturated $\left(r^{*}>14\right)$ SDSS sources. The distribution of a sparse sample of these sources on the sky is shown in the top panel in Figure 3. The spectroscopic data are available for a $774 \mathrm{deg}^{2}$ $\left(63^{\circ}\right)$ subregion and include spectra for $1.21 \times 10^{5}$ objects. The sky distribution of a sparse sample of sources with spectra is shown in the bottom panel in Figure 3. To test the robustness of analyzed quantities regarding the choice of area on the sky, we also use a smaller subsample based on four SDSS commissioning runs $(94,125,752$, and 756)

${ }^{26}$ Throughout this paper we use the term " $K$-correction" as it was defined by Schneider, Gunn, \& Hoessel (1983). taken during the fall of 1998 and the spring of 1999. These data are part of the publicly available SDSS Early Data Release $^{27}$ (see EDR) and include $6.68 \times 10^{6}$ unique unsaturated objects in a $325 \mathrm{deg}^{2}$ large region of sky bounded by $-1.25<\delta_{\mathrm{J} 2000.0}<1.25$ and either $0^{\mathrm{h}} 40^{\mathrm{m}}<\alpha_{\mathrm{J} 2000.0}<3^{\mathrm{h}}$

${ }^{27}$ Available at: http://www.sdss.org.
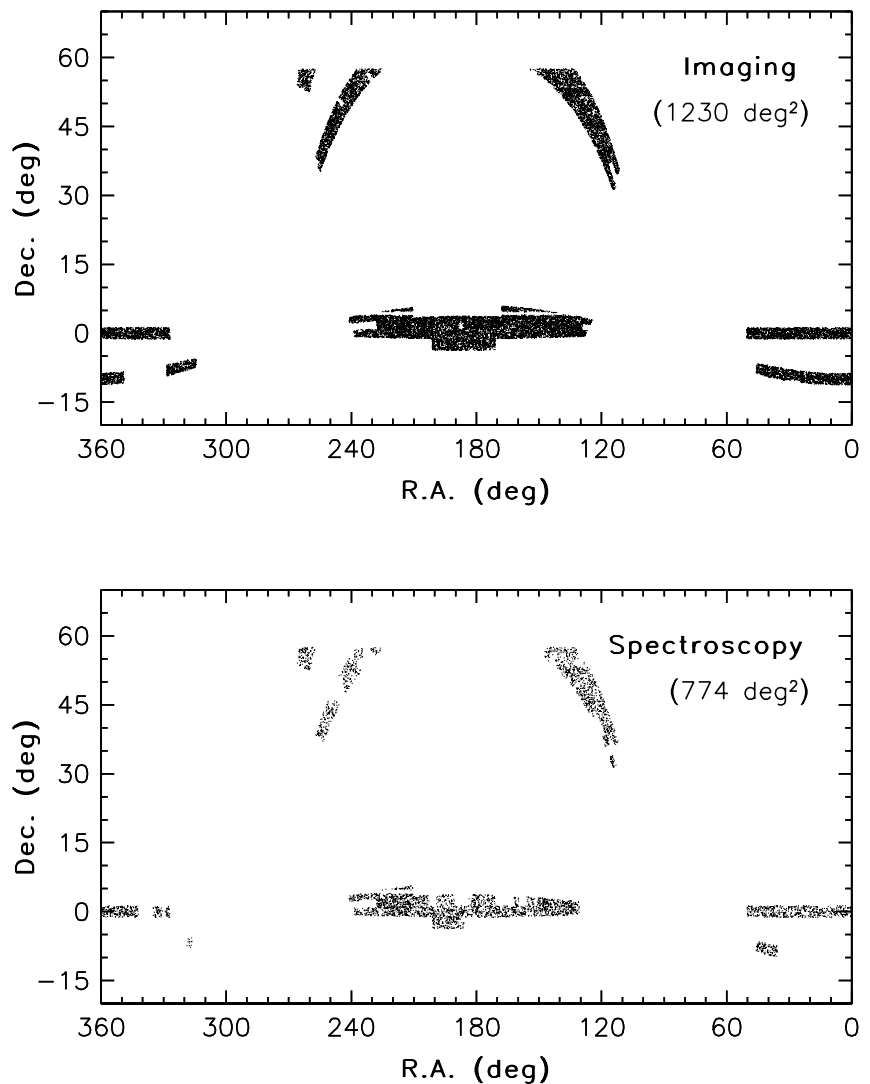

FIG. 3.-Top: Regions included in the SDSS imaging data studied here $\left(1230 \mathrm{deg}^{2}\right)$. A smaller region $\left(774 \mathrm{deg}^{2}\right)$ with SDSS spectroscopic data is shown in the bottom panel. The regions are outlined by sparse sampling the source positions. 
$20^{\mathrm{m}}$ (runs 94 and 125), or $9^{\mathrm{h}} 40^{\mathrm{m}}<\alpha_{\mathrm{J} 2000.0}<15^{\mathrm{h}} 40^{\mathrm{m}}$ (runs 752 and 756). Spectra are available for 38,000 objects from this smaller data set (the EDR sample hereafter).

Throughout the paper we introduce and describe various subsamples designed to avoid selection effects. A summary of these subsamples is provided in Appendix A.

\subsection{FIRST Data}

The full area $\left(1230 \mathrm{deg}^{2}\right)$ analyzed here includes 107,654 FIRST sources. The top panel in Figure 4 shows the $\log \left(\theta^{2}\right)$ versus $t$ diagram for the 28,476 sources in the EDR sample region. The radio emission from the sources above the $\log \left(\theta^{2}\right)=0$ line is resolved. The diagonal cutoff running from the top to the lower right corner is due to the FIRST faint limit; low surface brightness sources (i.e., those that are large and faint) are not included in the catalog. The differential $t$ distribution ("counts") for all sources is shown in the bottom panel with circles and separately with triangles for the 9823 sources with $\log \left(\theta^{2}\right)>0.1$ (note that this
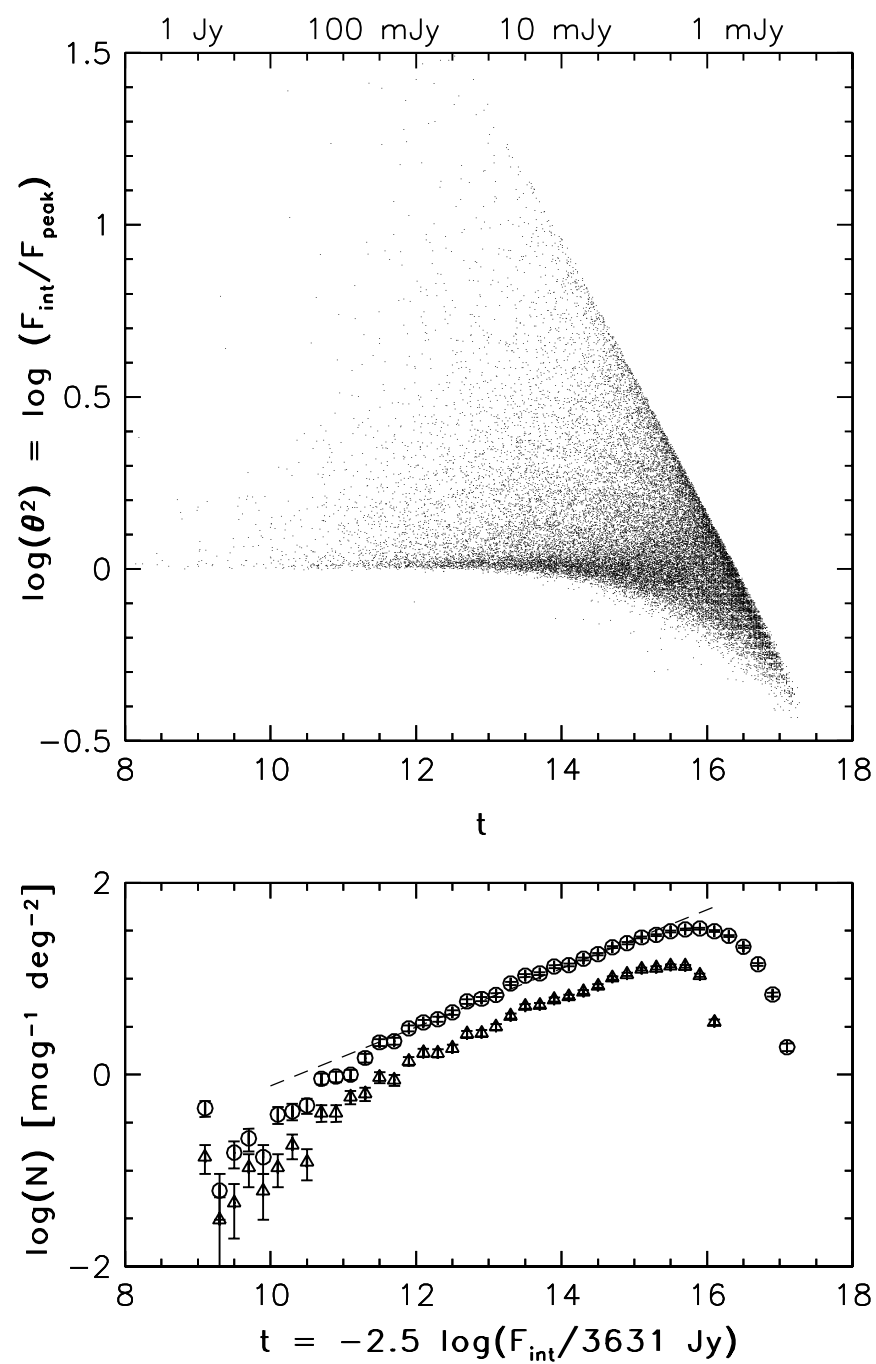

FIG. 4.-Top: Size measure, $\log \left(\theta^{2}\right)$, vs. the radio AB magnitude, $t$, for 28,476 FIRST sources from a $325 \mathrm{deg}^{2}$ large region of sky (EDR sample). The diagonal cutoff running from the top to the lower right corner is due to the FIRST faint limit. Bottom: Differential $t$ distributions ("counts") for all sources (circles), and for the 9823 sources with $\log \left(\theta^{2}\right)>0.1$ (triangles). The dashed line is a best linear fit to the counts of all sources in the $11.5<t<15.5$ range (see text). condition reliably selects resolved radio sources only for $t \lesssim 15$ [3.6 mJy] because noise affects the fainter sources). The counts suggest that the FIRST sample is complete for $t \lesssim 15.5(2.3 \mathrm{mJy})$. The differential counts $\left(\mathrm{mag}^{-1} \mathrm{deg}^{-2}\right)$ of all FIRST sources in the $11.5<t<15.5$ range can be described by

$$
\log (n)=-3.12+0.31 t
$$

This fit is shown by the dashed line and agrees well with the results discussed by Windhorst et al. (1985; for a related discussion see also White et al. 1997). The slope of the $\log (n)-t$ relation for sources with $\log \left(\theta^{2}\right)>0.1$ in the same $t$ range is statistically indistinguishable from the slope for the whole sample.

\subsection{The Positional Matching of SDSS and FIRST Catalogs}

We first positionally match all sources from both catalogs whose positions agree to better than $3^{\prime \prime}$, and find 37,210 such pairs. ${ }^{28}$ The distribution of the distance between the SDSS and FIRST positions, $d$, for the 10,084 pairs from the EDR subsample is shown in the top panel in Figure 5. In order to test whether the distance distribution depends on optical morphology, we split the EDR sample into 1999 optically unresolved and 8085 resolved sources (for a discussion of star-galaxy separation see $\S 3.6$ ). As is evident, the two distributions ${ }^{29}$ are similar.

The increase in the number of matches with $d \gtrsim 2$ ". 5 is consistent with expected random associations, given the number density of FIRST and SDSS sources (for details see Knapp et al. 2002, hereafter K02). Based on this histogram, we choose 1.15 as the limiting distance for a match to be considered as an optical identification, and we find 29,528 matches satisfying this criterion.

This cutoff is a trade-off between the completeness and contamination of the sample. For a cutoff at $3^{\prime \prime}$ practically all true matches (estimated to be 33,800 after subtracting the estimated number of random matches) are included in the sample, but the contamination from random matches is roughly $9 \%$. On the other hand, a cutoff at $1^{\prime \prime}$ with a contamination of $1.5 \%$ is only $72 \%$ complete. The chosen cutoff results in a $85 \%$ complete sample with a contamination of $3 \%$. The high completeness and low contamination are due to the excellent astrometric accuracy of both SDSS and FIRST. As a comparison, Magliocchetti \& Maddox (2002a) used a $2^{\prime \prime}$ cutoff for the APM-FIRST matches, and Sadler et al. (2002) used a $10^{\prime \prime}$ cutoff for the NVSS-2dFGRS matches.

Based on statistical considerations, the 29,528 optical identifications include $\sim 28,684$ true associations and $\sim 844$ random matches. The estimated completeness implies that, for the 107,654 FIRST sources, there are 33,746 SDSS counterparts, or $31 \%$ of all FIRST sources ${ }^{30}$ (of course, because of the completeness vs. contamination trade-off, robust identifications can be made only for $27 \%$ of FIRST sources). These identifications represent $\sim 0.14 \%$ of all SDSS sources in the analyzed region.

${ }^{28}$ This matching algorithm does not recognize the so-called core-jet, core-lobe, and double-lobe sources. Such sources are discussed in $\S 3.8$.

${ }^{29}$ All histograms marked as $n / N_{\text {tot }}$ are normalized such that the area under the curve is unity.

${ }^{30}$ The fraction of optically identified FIRST sources depends to some extent on SDSS observing conditions, particularly on seeing, which determines the SDSS imaging depth. 

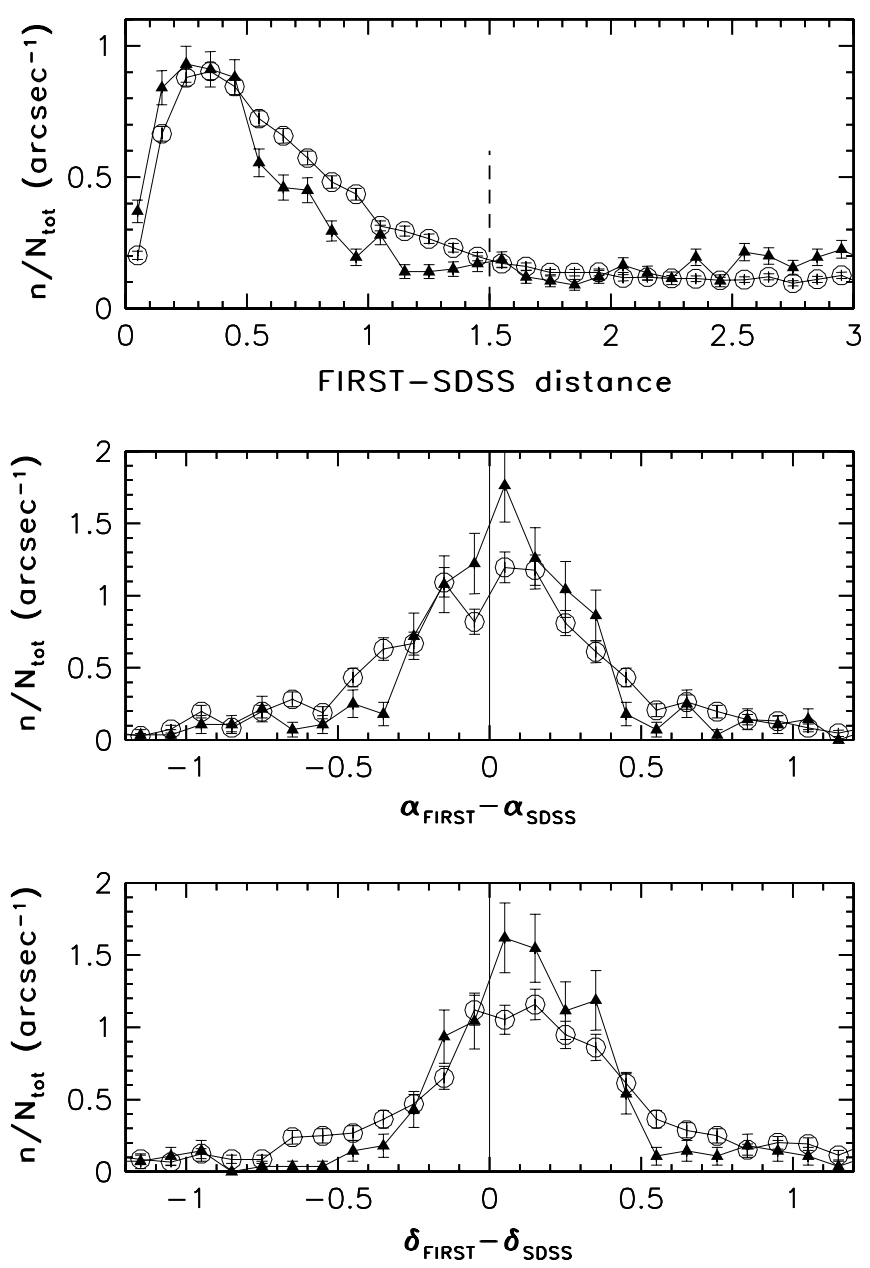

FIG. 5.-Top: Distributions of the distance between the SDSS and FIRST positions for the 10,084 close pairs from a $325 \mathrm{deg}^{2}$ region; the 1999 optically unresolved source are marked by triangles, and the 8085 resolved sources by circles. The vertical dashed line shows the adopted cutoff (1"5) for positional association that results in an $85 \%$ complete sample with a contamination of $3 \%$. The middle and bottom panels show differences in equatorial coordinates for sources with $r^{*}<20$ and $t<15$. The declination shows an offset of 0 ". 12 (see text).

\subsection{The Astrometric Accuracy of SDSS and FIRST Catalogs}

The sample discussed here is sufficiently large to determine systematic astrometric offsets between SDSS and FIRST catalogs. The middle and bottom panels in Figure 5 show the astrometric offsets in each equatorial coordinate for sources brighter than $r^{*}=20(36 \mu \mathrm{Jy})$ and $t=15(3.63$ $\mathrm{mJy})$ that are least affected by measurement noise. These histograms show a 0 ".045 offset in right ascension and 0 ". 120 offset in declination. Systematic offsets in the SDSS astrometric calibrations are thought not to exceed 0 ". $020-0$ ".030 (Pier et al. 2002). An additional 0".020 systematic error is present in PHOTO v5_2 astrometry as a result of the use of different centroiding algorithms in different pipelines (this will be eliminated in the next version of the pipelines and all subsequent data releases). Thus at most 0 ".050 of the offset may be attributable to systematics in the SDSS astrometry, implying similar systematic errors in the FIRST astrometry. This is an excellent agreement; for comparison, the FIRST and APM astrometric reference frames are offset by 0 ". 8 (Magliocchetti et al. 2000).
The equivalent Gaussian widths determined from the interquartile range $\left(q_{75}-q_{25}=1.335 \sigma\right)$ are 0 ".25 for unresolved sources, and 0 ". 35 for resolved sources (per coordinate; mean for both coordinates). As the multiple SDSS commissioning observations of the same area show that the positions of SDSS sources are reproducible to better than 0 ". $10 \mathrm{rms}$ per coordinate, the implied astrometric accuracy of the FIRST catalog is thus $\sim 0$ !.3 per coordinate for sources with $t<15$. When no limit on radio flux is imposed, the FIRST astrometric accuracy is $\sim 0$ ". 4 per coordinate. This is consistent with the FIRST claim that " the individual sources have $90 \%$ confidence error circles of radius less than 1 " at the survey threshold" (Becker et al. 1995).

\subsection{Matched versus Unmatched FIRST Sources}

Approximately $69 \%$ of FIRST sources do not have an SDSS counterpart within $3^{\prime \prime}$. Since the multiple SDSS scans of the same area, as well as matching to the 2MASS PSC sources (F00; Ivezić et al. 2002a) show that the SDSS completeness is better than $90 \%$ for $r^{*}<22$ (and approaching 99.3\% for $r^{*}<17.5$ ), the majority of unmatched FIRST sources are probably too optically faint to be detected in SDSS images. This conclusion is supported by deep imaging of a $1.2 \mathrm{deg}^{2}$ region in Hercules by Waddington et al. (2000). They identified 69 out of 72 FIRST sources from that region; all identified sources have $r^{*} \lesssim 26$, with the distribution maximum at $r^{*} \sim 22$. Although their sample is small, it is the most comprehensive nearly complete sample of optically identified radio sources at millijansky flux density levels.

We find no significant differences in the radio properties between FIRST sources with and without optical identifications. The top panel in Figure 6 compares the differential counts of FIRST sources from the EDR sample with an SDSS counterpart within $3^{\prime \prime}$ and those without as a function of radio $\mathrm{AB}$ magnitude, $t$. The two lines show best fits to the counts in the $11.5<t<15.5$ range: for unmatched sources

$$
\log (n)=-3.11+0.30 t
$$

and for matched sources

$$
\log (n)=-3.66+0.31 t,
$$

where $n$ is the number of sources per unit magnitude interval and square degree. These slopes are measured with an accuracy of $\sim 0.02$, and thus they are statistically identical; i.e., the optical identification probability does not depend on the radio flux for $t \gtrsim 11$.

The bottom panel in Figure 6 compares the distributions of $\log \left(\theta^{2}\right)$ for the 11,817 FIRST sources from the EDR sample with $t<15$ (the fainter sources suffer from low signal-to-noise ratio; see Fig. 4). The number of sources decreases faster with $\theta$ for the optically identified than for unidentified radio sources, implying that the optical identification probability is somewhat lower for the radio resolved sources.

\subsection{The Star-Galaxy Classification 3.6.1. Morphological Classification}

The SDSS photometric pipeline classifies detected sources into resolved and unresolved objects (see $\S 2.1$ and EDR). In its current implementation, the photometric pipeline uses a binary classification: an object is either a "star" (unre- 

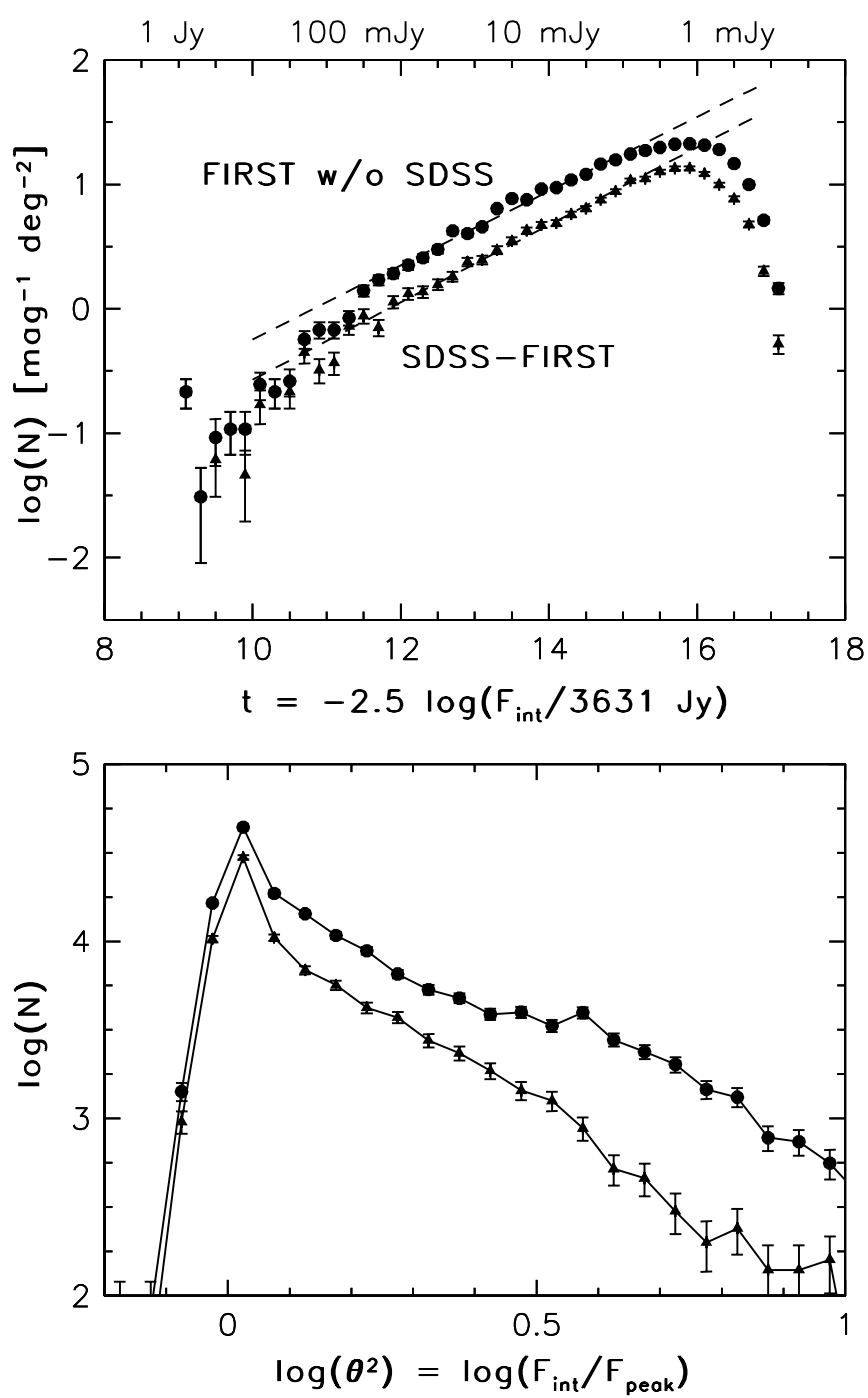

FIG. 6.-Top: Comparison of the differential counts of FIRST sources with an SDSS identification (triangles) to those without (dots), as a function of radio $\mathrm{AB}$ magnitude, $t$. The two lines show best linear fits to the counts in the $11.5<t<15.5$ range. Bottom: Comparison of the distributions of $\theta$, which is a rough measure of the source radio size, for sources with $t<15$ (same notation as top).

solved) or a "galaxy" (resolved). Multiple SDSS scans, comparison with the HST data, and the distribution of sources in color-color diagrams show that the star-galaxy separation is reliable to better than $90 \%$ for sources with $r^{*} \sim 21$ and to better than $95 \%$ for sources with $20<r^{*}<21$ (Lupton et al. 2002). This can be seen qualitatively in the bottom two panels in Figure 1, where the color distributions of unresolved and resolved sources are markedly different even at the faint end (colors are not used in the classification).

We chose the $r^{*}<21$ condition to define subsamples with robust star-galaxy separation, resulting in 18,908 sources (out of 29,528), classified as $3225(17 \%)$ unresolved and $15,683(83 \%)$ resolved sources. For brevity, in the remainder of this work we will call optically resolved FIRST sources galaxies and optically unresolved FIRST sources quasars. While there may be some optically resolved FIRST sources that are not galaxies (e.g., Galactic supernova remnants) or optically unresolved FIRST sources that are not quasars (e.g., stars with radio emission, see K02 and references therein), their numbers in the sample discussed here are expected to be insignificant.

\subsubsection{Color Classification}

The color distributions of optically unresolved and resolved SDSS-FIRST sources are very different. This difference is especially large in the $r^{*}-z^{*}$ color. The top panel in Figure 7 shows the $r^{*}$ versus $r^{*}-z^{*}$ color-magnitude diagram for the 29,528 optically identified FIRST sources. It is evident that the $r^{*}-z^{*}$ color is a good separator of the two morphological types, with the optimal cut depending on the $r^{*}$ magnitude: the unresolved sources are blue and the resolved sources are red. The separation is clean even at the faintest levels in the diagram. The bottom panel shows the $r^{*}-z^{*}$ distributions for sources with $21<r^{*}<21.5$.

This good correlation between the morphology and color can be used to estimate an upper limit on the fraction of sources with incorrect morphological classification. We assume that all quasars are blue and all galaxies are red, and interpret sources with "incorrect" color as misclassified. Adopting a cut $r^{*}-z^{*}=1.0$ for sources with $21<r^{*}<21.5$, we find that $20 \%$ of selected quasars have $r^{*}-z^{*}>1.0$ and $26 \%$ of selected galaxies have $r^{*}-z^{*}<1.0$. Adopting the same $r^{*}-z^{*}$ cut for sources with
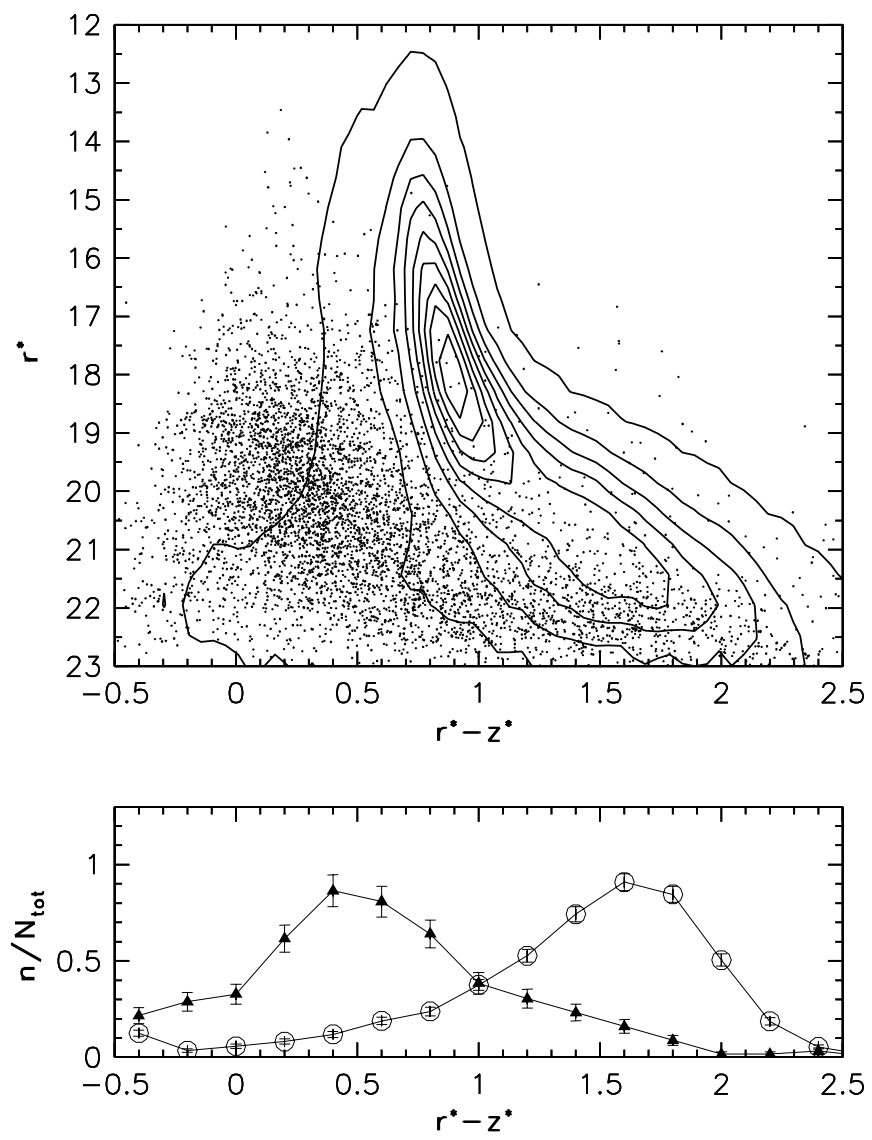

FIG. 7.-Top: The $r^{*}$ vs. $r^{*}-z^{*}$ color-magnitude diagram for the optically identified FIRST sources. The 23,898 resolved sources are shown as contours and 5623 unresolved sources as dots. Bottom: The $r^{*}-z^{*}$ distributions for sources with $21<r^{*}<21.5$; the triangles correspond to 625 unresolved sources, and the circles to 2437 resolved sources. Optically resolved sources tend to be red, while unresolved sources tend to be blue, even close to the faint limit. 
$21.5<r^{*}<22$, we find that the fractions of objects with "incorrect" color are still smaller than $25 \%$. This is a robust upper limit on the inaccuracy of the adopted star-galaxy separation at the faint end. Of course, some of the objects with "incorrect" color may be correctly classified (e.g., high-redshift quasars could have $r^{*}-z^{*}>1.0$; see Richards et al. 2002).

\subsection{The Radio Properties of SDSS-FIRST Sources}

The radio properties of the galaxies and quasars brighter than $r^{*}=21$ are shown in Figure 8. The top panel shows the differential counts in radio magnitude. The two lines show best fits to the counts in the $11.5<t<15.5$ range: for quasars

$$
\log (n)=-2.24+0.14 t
$$
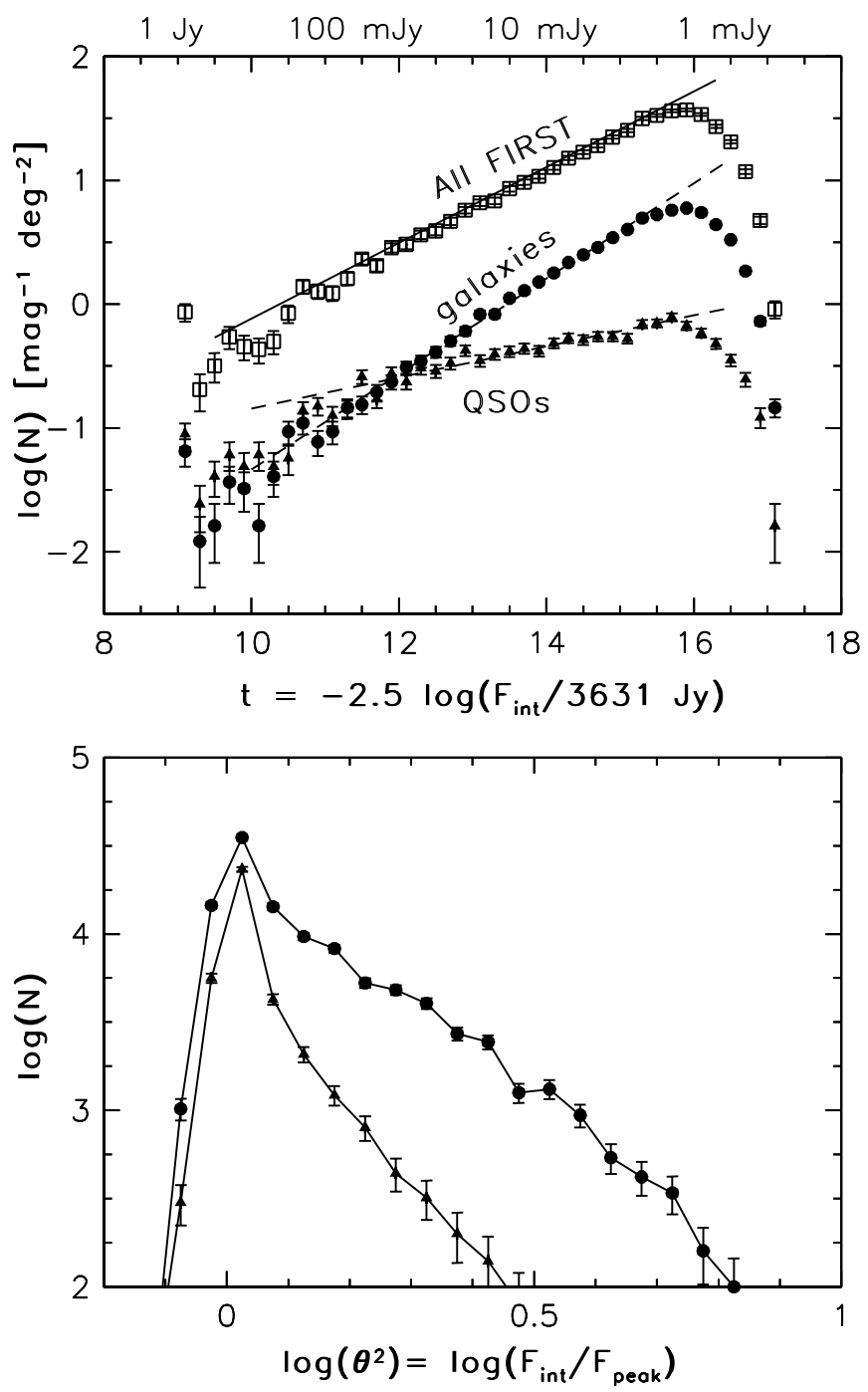

FIG. 8. - Comparison of the radio properties for SDSS-detected quasars and galaxies. Top: Comparison of the differential counts as a function of the radio magnitude for 3142 quasars (dots) and 16,109 galaxies (triangles). The dashed lines show the best fits discussed in text. The sum of counts for quasars and galaxies, multiplied by 5.6 to account for the matching fraction, is shown as open squares and compared with the counts of all FIRST sources, shown by the solid line. Note the similarity between the two distributions. Bottom: Distributions of the radio size parameter $\theta$ for quasars (triangles) and galaxies (circles) with $t<15$. and for galaxies

$$
\log (n)=-5.33+0.40 t,
$$

where $n$ is the number of sources per unit magnitude interval and square degree. These fits imply that, for $r^{*}<21$, the fraction of quasars in the FIRST catalog is a strong function of the radio flux, monotonically decreasing from $\gtrsim 50 \%$ for bright radio sources to $\lesssim 10 \%$ at the FIRST sensitivity limit. As discussed above, the cumulative quasar fraction among the SDSS-FIRST sources is $17 \%$. These results are in qualitative agreement with those of Magliocchetti \& Maddox (2002a) based on the FIRST-APM matching (for earlier results, see Windhorst et al. 1985 and references therein).

The number counts versus magnitude slope of 0.31 for all identified sources (eq. [8)] is simply a mean relation resulting from the mixing of two different populations: quasars with a slope of 0.14 , and galaxies with a slope of 0.40 . Since optically identified and unidentified FIRST sources have the same number counts slope, it is plausible that the fractions of quasars and galaxies are roughly the same for the two subsamples. To further illustrate this point, we add the counts of quasars and galaxies, multiply them by 5.6 to account for the fraction of sources that are matched $(17.9 \%$ of FIRST sources pass the cuts on maximum positional discrepancy, optical brightness and robust optical classification), and compare them to the counts of all FIRST sources. The squares in the top panel in Figure 8 show the scaled counts for optically identified sources, and the solid line shows the best-fit to the counts of all FIRST sources, as discussed in $\S 3.2$. The similarity of the two distributions supports the notion that the fractions of quasars and galaxies are roughly the same for the matched and unmatched FIRST sources (these fractions are 17\% quasars and 83\% galaxies when no radio flux limit is imposed, and 26\% quasars and $74 \%$ galaxies for $t<15$ ). We will return to this point in Appendix B where we discuss the limits on the number of quasars missed in optical surveys.

The bottom panel in Figure 8 displays the distributions of the radio concentration measure $\log \left(\theta^{2}\right)$ for galaxies and quasars, where we count only the sources with $t<15$. Note that galaxies tend to have larger radio sizes (i.e., the radio emission is resolved on scale of $\sim 5^{\prime \prime}$ ), which suggests that a significant fraction of their radio emission either originates outside their nucleus or that double-lobe radio emission is resolved (for a related discussion, see $\S 5.2 .4$ ). Out of 6646 matched galaxies with $t<15$, there are $2486(37 \%)$ with $\log \left(\theta^{2}\right)>0.1$, and $3374(51 \%)$ with $\log \left(\theta^{2}\right)>0.05$. For comparison, out of 2133 matched quasars with $t<15$, there are $296(14 \%)$ with $\log \left(\theta^{2}\right)>0.1$, and $520(24 \%)$ with $\log \left(\theta^{2}\right)>0.05$. Thus the fraction of quasars with resolved radio emission is significantly lower than the fraction of galaxies with resolved radio emission.

\subsection{Sources with Complex Radio Morphology}

In preceding sections we have only discussed sources for which the radio and optical positions agree to better than 1".5 (hereafter core sources). This sample does not address the so-called core-jet sources (optical-radio match with an additional nearby radio source), core-lobe (optical-radio match with two nearby radio sources at similar distances from the core), and double-lobe radio sources (similar to core-lobe sources except that there is no radio source in the middle). Such sources were first discussed in the context of 
FIRST data by Magliocchetti et al. (1998) and McMahon et al. (2001). Here we describe two methods for recognizing radio sources with complex morphology using SDSS and FIRST data and compare their number with the number of optical-radio identifications described in $\S 3.3$. For this analysis we use only the EDR sample (see $\S 3.1$ ). The full sample will be discussed in a separate publication (Ivezić et al. 2002b, hereafter Paper II).

An estimate of the importance of core-jet, core-lobe, and double-lobe sources relative to simple one-on-one opticalradio matches can be illustrated by considering the distribution of the nearest neighbor distances for FIRST sources. McMahon et al. (2001) showed that, for isolated ${ }^{31}$ FIRST sources (their Fig. 14), there is an excess of nearest neighbors closer than about $1^{\prime}$ over the number expected for a random source distribution. We matched SDSS-FIRST sources with the nearest FIRST neighbor without an SDSS match and found the same result. In the top panel in Figure 9 we compare the nearest neighbor distance distribution for FIRST sources matched with an optically resolved SDSS source with that obtained for the same number of random positions. The number of nearest neighbors closer than $30^{\prime \prime}$ is about 10 times larger than that expected for a

${ }^{31} \mathrm{McMahon}$ et al. defined isolated pairs of sources as those without a third source within $2^{\prime}$.

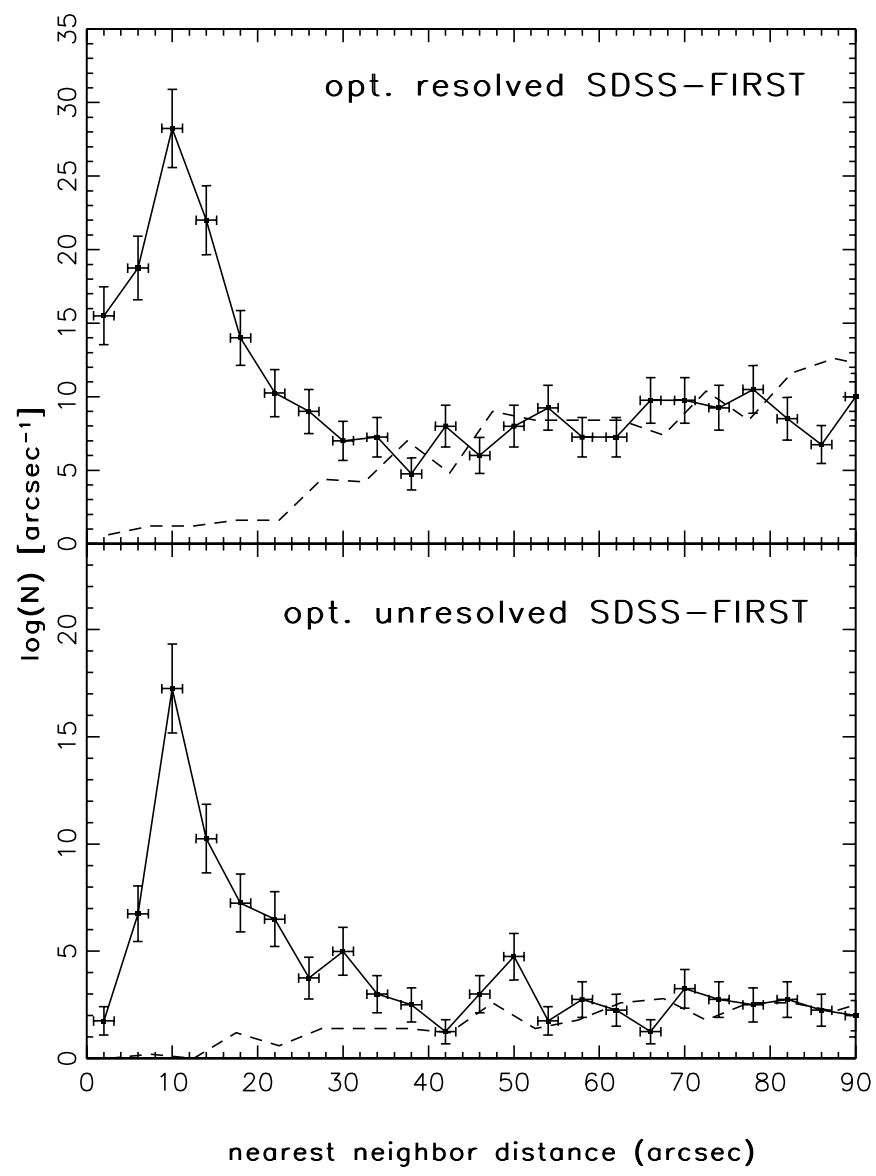

FIG. 9.-Distribution of distance between SDSS-FIRST sources, as marked, and their nearest optically unidentified radio neighbor (solid lines). Nearest optically unidentified radio neighbor distance distribution for the same number of random positions (dashed lines). random source distribution. The bottom panel shows analogous distributions for optically unresolved sources. The surface density of optically resolved SDSS-FIRST sources with the nearest neighbor closer than $30^{\prime \prime}$ is 1.3 $\mathrm{deg}^{-2}$ and for unresolved sources $0.64 \mathrm{deg}^{-2}$ (accounting for contamination by random associations).

These sources are good candidates for radio sources with complex morphology and represent $\lesssim 10 \%$ of the SDSSFIRST surface density for core sources. In addition to considering the nearest FIRST neighbors, we also develop a technique for finding double-lobe sources, described in the next section. We use these two methods to estimate the fractions of core, core-jet, core-lobe, and double-lobe among optically identified radio sources.

\subsubsection{Core-Lobe and Double Lobe Sources}

We find core-lobe ${ }^{32}$ and double-lobe sources by computing the midpoint for each of the 13,146 unique nearest neighbor pairs among unmatched FIRST sources ${ }^{33}$ and rematching these midpoints to the SDSS catalog. We initially use a large matching radius of $6^{\prime \prime}$ and do not place any constraints on the neighbor distance and the optical properties of matched sources, resulting in 3197 matches. The distribution of the neighbor distances, the distances between the radio midpoint and optical position, and their comparison with random distributions indicate that the majority of matches are random associations. By studying the distribution of the matched sources in optical color-color and colormagnitude diagrams, we determined that the fraction of random matches can be significantly decreased without much effect on the completeness of the matched sample by selecting only FIRST neighbors closer than $90^{\prime \prime}$, and optical-midpoint distance smaller than $3^{\prime \prime}$. These selection criteria and the $r^{*}<21$ requirement to ensure robust stargalaxy separation result in 74 optically unresolved and 239 optically resolved matches. Of those, 27 and 41, respectively, are already in the core sample and thus represent core-lobe sources. The estimated random association number is 16 for the unresolved and 28 for the resolved sample. These estimates are consistent with those obtained by the visual inspection of 313 FIRST $2^{\prime} \times 2^{\prime}$ images centered on midpoints. The top two rows in Figure 10 show examples of core-lobe and double-lobe sources recognized by this selection algorithm. It is reassuring that almost identical samples can be obtained without any limits on the neighbor distance and the matching distance, but instead using the appropriate cuts in optical color space (for more details see Paper II).

The importance of core-lobe and double-lobe sources can be gauged by comparing the above numbers with the matching results from preceding sections. In the same area there are 1094 optically unresolved and 5340 optically resolved SDSS-FIRST matches brighter than $r^{*}=21$ (accounting for the sample contamination and incompleteness). Of those, 27 and 41, respectively, are core-lobe sources and an additional 31 and 170, respectively, are double-lobe sources (accounting for contamination by random associations). That is, core-lobe and double-lobe sources together repre-

\footnotetext{
${ }^{32}$ Core-lobe and core-jet sources are included in the sample obtained by simple matching within 1 1"5. However, they are not recognized as such.

${ }^{33}$ There are 19,027 FIRST sources in the EDR area without an SDSS counterpart within $3^{\prime \prime}$. Because two sources can be the nearest neighbor to each other, the number of unique nearest neighbor pairs is 13,146 .
} 


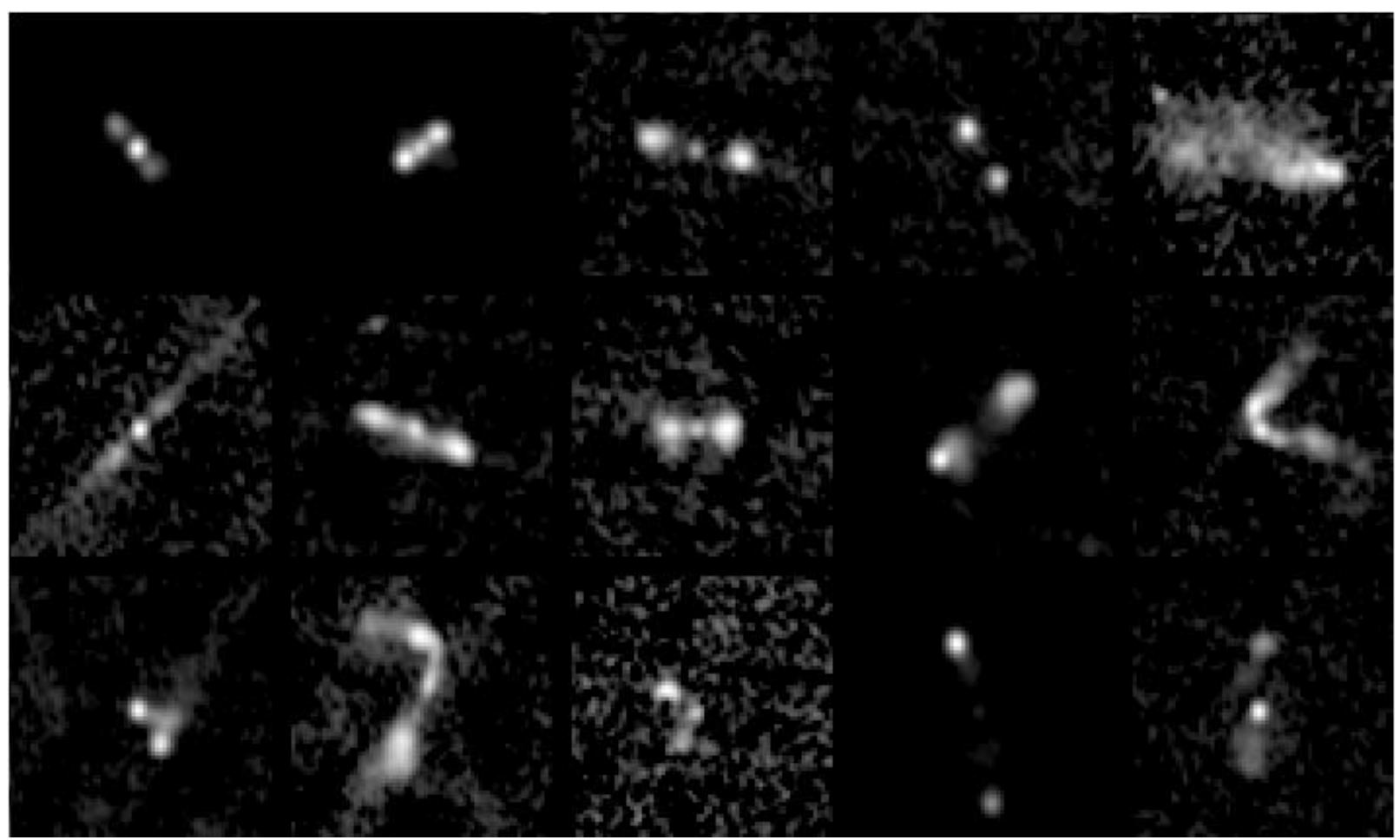

FIG. 10.-Examples of FIRST sources with complex morphology recognized by the selection algorithm. Each FIRST stamp is $2^{\prime} \times 2^{\prime}$, normalized to the maximum intensity and displayed with a square root stretch from 0.01 to 1 . Top and middle: core-lobe and double-lobe sources matched to optically unresolved and resolved SDSS sources, respectively, and ordered by the lobe-to-core radio flux ratio. The last column shows examples of sources that are more complex than simple core-lobe and double-lobe paradigms. Bottom: Bent core-lobe sources. The first three examples are associated with optically resolved sources, and the last two examples with unresolved sources. [See the electronic edition of the Journal for a color version of this figure.]

sent only about $5 \%$ of all optical-radio associations. It is noteworthy that the number ratio of double lobe to corelobe sources is about 1 for optically unresolved and about 4 for optically resolved SDSS-FIRST matches.

\subsubsection{Core-Jet Sources and Bent Core-Lobe Sources}

An SDSS-FIRST source with a single radio lobe would be missed by the above algorithm. While such a source would be selected by the simple positional one-to-one match, the existence of a nearby lobe would be unrecognized. To place an upper limit on the number of such sources, we searched for the two nearest FIRST sources without an SDSS match to each of the 6434 matched SDSS-FIRST sources brighter than $r^{*}=21$. Constraining the sample to sources with the nearest neighbor within $90^{\prime \prime}$ and the second nearest neighbor within $300^{\prime \prime}$, we select 678 sources. From these 678 sources we select 453 candidates for core-jet sources by requiring that the distance to the second nearest neighbor is at least $10^{\prime \prime}$ away and more than $20 \%$ larger than the nearest neighbor distance. This particular cut is motivated by the detailed distribution of sources in the plane spanned by the distances to the nearest two neighbors, and it essentially selects sources where the two neighbors are not at similar distances. The remaining 225 sources are treated as candidates for bent double-lobe sources (see below).

The 453 candidates for core-jet sources include 78 optically unresolved and 375 resolved sources. Repeating the same analysis for random sets of positions produces samples of 51 and 235 candidates and indicates that the surface density of core-jet sources is not larger than about 0.08 and
$0.43 \mathrm{deg}^{-2}$ for unresolved and resolved sources, respectively. Thus the upper limit for the fraction of core-jet sources is about $2.5 \%$.

In $\S 3.8 .1$ we searched for optical matches close to the midpoint of the line connecting two radio lobes. However, in the so-called "bent-double radio sources" the optical match can be significantly offset from the radio midpoint (e.g., Blanton et al. 2001 and references therein), and our algorithm thus may be missing such sources. To place an upper limit on the number of bent core-lobe sources, we consider as candidates the 225 matched SDSS-FIRST sources with similar nearest-neighbor pairs described above, which include 56 optically unresolved and 169 resolved sources. This sample includes the 68 core-lobe sources discussed in the previous section (i.e., "straight" core-lobe sources) but does not include double lobe sources because they do not have core radio emission. The same algorithm applied to a set of random positions yields samples of 14 and 70 sources, placing an upper limit for the core-lobe source surface density of $0.13 \mathrm{deg}^{-2}$ for quasars and 0.30 $\mathrm{deg}^{-2}$ for galaxies.

The visual inspection of $2^{\prime} \times 2^{\prime}$ FIRST images indicates that the number of convincing bent core-lobe cases is less than about $20 \%$ of the number of "straight" core-lobe sources recognized by the midpoint matching. That is, for the majority of core-lobe sources the optical match is close to the midpoint. Examples of identified bent core-lobe sources are shown in the bottom row in Figure 10. It is noteworthy that the majority of bent core-lobe sources are associated with optically resolved sources, in agreement with Blanton et al. (2001). 
To summarize, the fraction of SDSS-FIRST sources with complex radio morphology is less than $10 \%$. For optically resolved sources double lobe morphology is the dominant type, while for unresolved sources the fractions of core-lobe and double lobe morphological types appear comparable. We will limit the rest of our discussion to the core sources (including together core-only, core-jet, and core-lobe sources) since they form the overwhelming majority of the SDSS-FIRST sample. The optical and radio properties of the lobe sources will be discussed in more detail in Paper II.

\subsection{The Incompleteness and Contamination of the FIRST Catalog Determined from the Comparison with the NVSS Catalog}

Becker et al. (1995) showed, by comparing FIRST and NRAO VLA Sky Survey (NVSS; Condon et al. 1998 catalogs, that radio sources larger than about $10^{\prime \prime}$ are overresolved by FIRST and have underestimated flux. Nevertheless, the fluxes of sources that appear unresolved in both surveys (the NVSS resolution is $45^{\prime \prime}$ ) agree well over 3 orders of magnitude. In their comparison Becker et al. considered only isolated FIRST sources (those whose nearest neighbor is at least $100^{\prime \prime}$ away) and did not use the NVSS catalog to estimate the incompleteness and contamination of the full FIRST catalog. Accurate estimates of completeness are difficult to obtain because of the factor of 8 difference in angular resolution; in particular, large NVSS sources may appear as several FIRST components. In some cases these components may simply be distinct sources that were unresolved by NVSS, but they can also represent a single complex source that was split by the FIRST source extraction algorithm.

We determine approximate upper limits for the FIRST incompleteness and contamination by positionally matching the 28,476 FIRST sources and 17,990 NVSS sources from the EDR area (see $\S 3.1$ ). The distribution of the distances $^{34}$ between the FIRST and NVSS positions indicates that a $15^{\prime \prime}$ cutoff includes practically all real matches, while keeping the random association rate under $1 \%$. Adopting this cutoff results in 15,700 matches, or $87 \%$ of the NVSS sources (the fraction of matched FIRST sources, 55\%, is lower because FIRST is deeper than NVSS). Thus the incompleteness of the FIRST catalog is not larger than 13\% and may be significantly smaller if most of the missing NVSS sources are nearby distinct sources that FIRST resolved.

The radio magnitude distribution of FIRST sources with and without an NVSS match is shown in Figure 11, for FIRST sources with an SDSS counterpart in the top panel and for the remaining sources in the bottom panel. For sources brighter than the NVSS completeness limit, $t \lesssim 15$, $94.4 \%$ of SDSS-FIRST and $83 \%$ of FIRST-only sources have an NVSS counterpart. Equivalently, out of 11,856 FIRST sources with $t<15,1588$ do not have an NVSS match within $15^{\prime \prime}$. Assuming that all FIRST sources without an NVSS match are spurious, we determine the upper limit for the contamination of the FIRST catalog for $t<15$ of $13 \%$. Of course, a significant fraction of these are distinct sources resolved by FIRST and not by NVSS.

\footnotetext{
34 The FIRST-NVSS R.A. and decl. differences appear to follow an exponential distribution, $\exp \left(-|d| / 3^{\prime \prime}\right)$, rather than a Gaussian distribution.
}
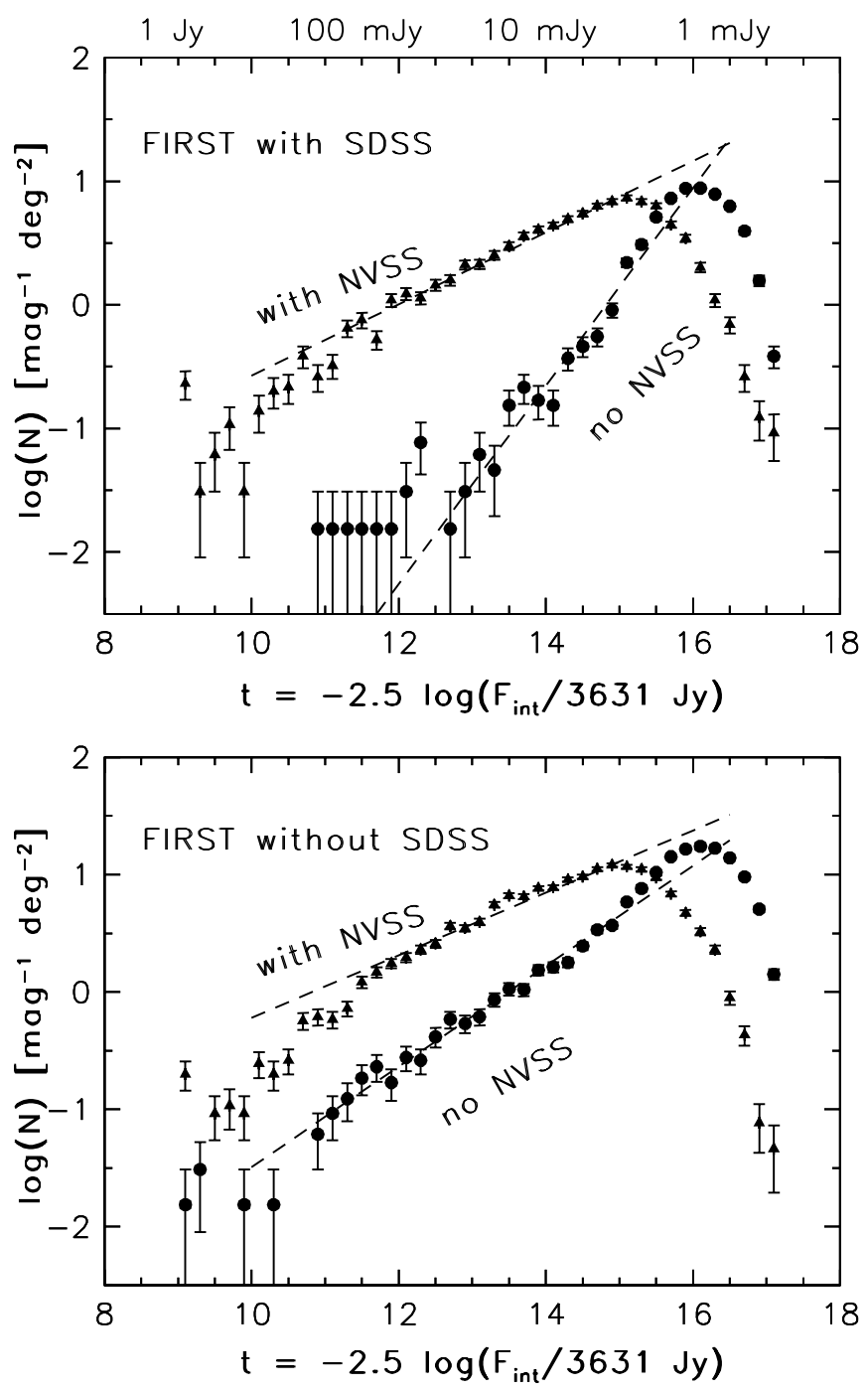

FIG. 11.-Radio magnitude distribution of FIRST sources with and without an NVSS match, as marked. Top: SDSS-FIRST sources. Bottom: FIRST sources without an SDSS match.

We have visually inspected FIRST stamps for 42 SDSSFIRST sources without NVSS counterparts and $13<t<14$ and for 138 FIRST-only sources with $13<t<13.5$. In more than $80 \%$ of these cases the FIRST source is a resolved double source, with the distance between the components larger than $30^{\prime \prime}$. Because of the lower NVSS resolution, these sources were not resolved, and thus the NVSS centroid is between the two components and more distant than the matching radius from each of the centroids measured by FIRST. The conclusion, that most of the mismatch between FIRST and NVSS for $t<15$ is a result of FIRST resolving double sources, is supported by the counts of mismatched sources, as shown in Figure 12. For $t<15$ there are roughly twice as many FIRST sources (1588) without an NVSS match as there are NVSS sources without a FIRST match (756), and this ratio seems to be nearly independent of flux.

The top panel in Figure 13 shows the magnitude difference between the FIRST and NVSS measurements as a function of the magnitude measured by FIRST for the 5757 matched sources with an SDSS counterpart. The analogous diagram for FIRST sources without an SDSS counterpart 


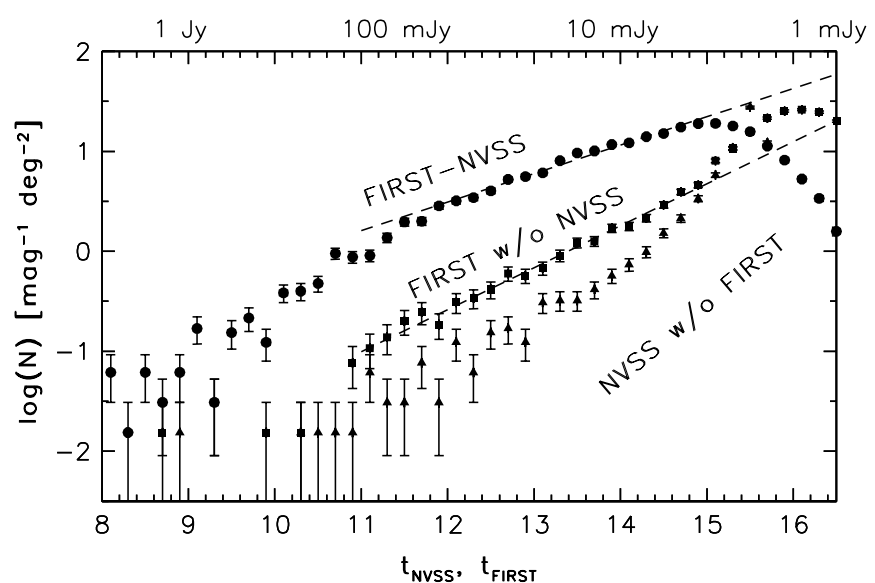

FIG. 12.-Radio magnitude distribution of FIRST-NVSS sources matched within $15^{\prime \prime}$ (circles), FIRST sources without an NVSS match (squares), and NVSS sources without a FIRST match. The first two distributions are plotted as a function of FIRST flux, and the last one as a function of NVSS flux.
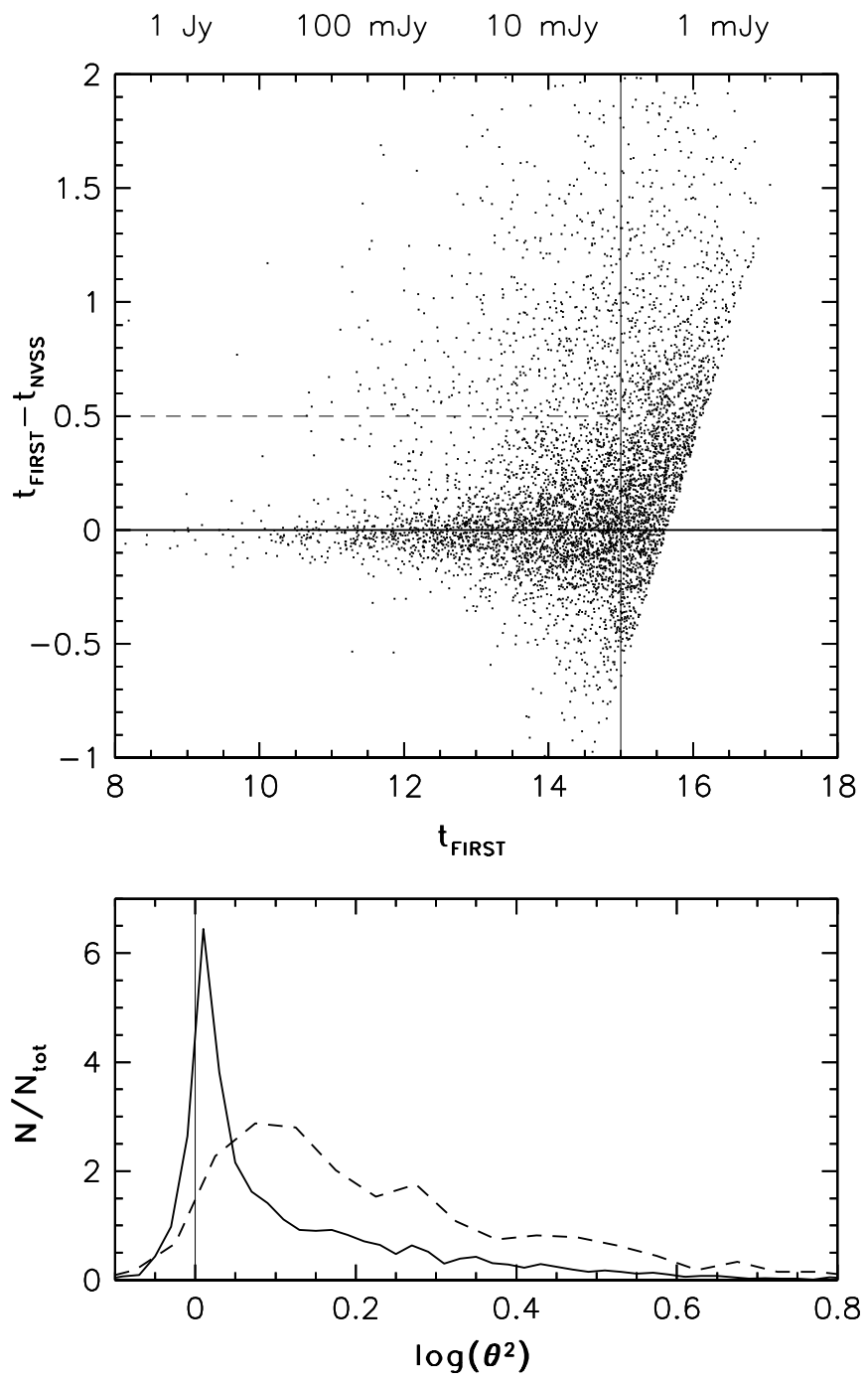

FIG. 13.-Top: Difference in $20 \mathrm{~cm}$ radio magnitudes measured by FIRST and NVSS as a function of FIRST magnitude for sources with SDSS counterpart. Bottom: Distribution of $\theta$, which is a rough measure of the source radio size, for all sources with $t<15$ by the solid line, and for the subset of those with $t_{\mathrm{FIRST}}-t_{\mathrm{NVSS}}>0.5$ by the dashed line. looks similar. The FIRST flux can be underestimated by up to 2 mag, though only for a small fraction of sources (for $t_{\mathrm{FIRST}}<15,15 \%$ of sources have $t_{\mathrm{FIRST}}-t_{\mathrm{NVSS}}>0.5$ ). The solid line in the bottom panel shows the $\theta$ distribution (measured by FIRST; see $\S 2.2$ ) for sources with $t<15$, and the dashed line shows the $\theta$ distribution for the subset with $t_{\text {FIRST }}-t_{\text {NVSS }}>0.5$. Most of the sources with large magnitude discrepancies are resolved by FIRST, in agreement with the analysis by Becker et al. (1995).

We conclude that incompleteness, contamination, and the magnitude bias for large sources in the FIRST catalog, while not negligible, are all insufficiently large to significantly affect the results discussed in subsequent sections.

\section{THE OPTICAL AND RADIO PROPERTIES OF SDSS-FIRST QUASARS}

In this section we analyze optical colors and counts and the distribution of radio-to-optical flux ratio for the 3225 optically unresolved SDSS-FIRST sources; spectra are available for a subsample of 1154 objects. A control sample of 20,085 spectroscopically confirmed SDSS quasars ${ }^{35}$ is used where appropriate. We estimate the fraction of radio quasars with stellar colors, argue that the data analyzed here support the existence of the quasar radio dichotomy, discuss a color difference between radio-loud and radio-quiet quasars, and demonstrate that the slopes of optical counts versus magnitude relations for radio-loud and radio-quiet quasars are indistinguishable for $i^{*}<18$.

Although the SDSS-FIRST quasars are dominated by low-redshift $(z \lesssim 2.5)$ objects, the sample also includes some high-redshift objects. In a sample of 462 SDSS quasars at redshifts greater than $3.6,17$ objects $(3.7 \%)$ are detected by FIRST, representing $1.5 \%$ of the spectroscopically confirmed SDSS-FIRST quasars. The highest-redshift object is SDSSp J083643.85+005453.3 with a redshift of 5.82 (Fan et al. 2001).

\subsection{The Optical Colors of FIRST Quasars}

One of the most important advantages of a radio-selected sample of quasars is that it suffers neither from dust extinction nor confusion with stars. ${ }^{36}$ Thus such samples can be used to estimate a fraction of quasars with stellar colors that are missed by optical surveys, such as SDSS, and an upper limit for the number of quasars with such a large extinction that they are undetectable at optical wavelengths. Such analysis assumes that the color distribution of radio quasars is similar to the distribution for the whole sample and, in particular, that the fraction of radio quasars with stellar colors is representative of the whole sample. Although we show in $\S 4.1 .2$ that the color distribution of radio quasars is different from that for the whole sample, the difference is sufficiently small that it does not significantly affect the conclusions of this section.

\footnotetext{
${ }^{35}$ For a detailed discussion of quasar classification from SDSS data, see Schneider et al. (2002).

${ }^{36} \mathrm{~K} 02$ show that some optically unresolved SDSS-FIRST sources are genuine radio stars. However, their number is very much smaller than the number of quasars
} 


\subsubsection{The Fraction of Quasars with Stellar Colors}

The majority of optically unresolved SDSS-FIRST sources have nonstellar colors (for a discussion of quasar colors in the SDSS photometric system see Richards et al. 2001). We determine the fraction of sources with colors indistinguishable from those of stars using the following procedure ${ }^{37}$ First we define a flux-limited sample of 2318 optically unresolved matched objects with $r^{*}<20.5$. This sample is then divided into three subsamples, which have all four, three, and only two reliable SDSS colors as a result of noise at the faint end. By adopting stellar locus masks ${ }^{38}$ (Fig. 14, dashed lines), we count all sources that cannot be distinguished from stars using available colors (for a source to be considered inside the stellar locus, it must be inside the locus in all two-dimensional color projections). The masks

${ }^{37}$ Since the sample discussed here has a fainter optical flux cutoff than objects targeted for SDSS spectroscopy, we decided not to use the quasar targeting pipeline (Richards et al. 2002) to define the stellar locus, because it is tuned for sources with $i^{*}<19$ for redshifts below 3 .

${ }^{38}$ By design, the stellar locus masks used here include some sources that the quasar targeting pipeline recognizes as outliers from the stellar locus; that is, the conservative approach adopted here slightly overestimates the number of sources with stellar colors. allow for up to $0.15 \mathrm{mag}$ distance from a best-fit to the stellar locus in each color-color diagram, except for the vertical part of the mask in the $g^{*}-r^{*}$ versus $u^{*}-g^{*}$ diagram, where the maximum allowed distance is $0.3 \mathrm{mag}$.

For the 1900 objects in this sample with $u^{*}<21$, all four SDSS colors are accurate to better than $\sim 0.1$ mag. We found that 75 of these $(3.9 \% \pm 0.5 \%)$ have colors indistinguishable from stars. Of the remaining 1825 objects with nonstellar colors, 1666 show strong UV color excess $\left(u^{*}-g^{*}<0.7\right)$. Sources with $u^{*}>21$ can be divided into 340 objects with $g^{*}<21$ and 78 objects with $g^{*}>21$. From the $r^{*}-i^{*}$ versus $g^{*}-r^{*}$ and $i^{*}-z^{*}$ versus $r^{*}-\mathrm{i}^{*}$ color-color diagrams we found that 179 of the former have stellar colors, and by using the $i^{*}-z^{*}$ versus $r^{*}-i^{*}$ color-color diagram we found that 49 of the latter have colors indistinguishable from stellar. In summary, 303 objects $(13 \% \pm 1 \%$ of the sample) cannot be distinguished from stars by using colors alone; for objects with $u^{*}<21$ this fraction is $4 \%$. We obtain consistent results for a subsample with $i^{*}<19$ (the SDSS spectroscopic targeting cutoff for low-redshift quasars) and when we consider only the EDR subsample.

To illustrate these cuts, the top left panel in Figure 14 shows the $u^{*}$ versus $u^{*}-g^{*}$ color-magnitude diagram for
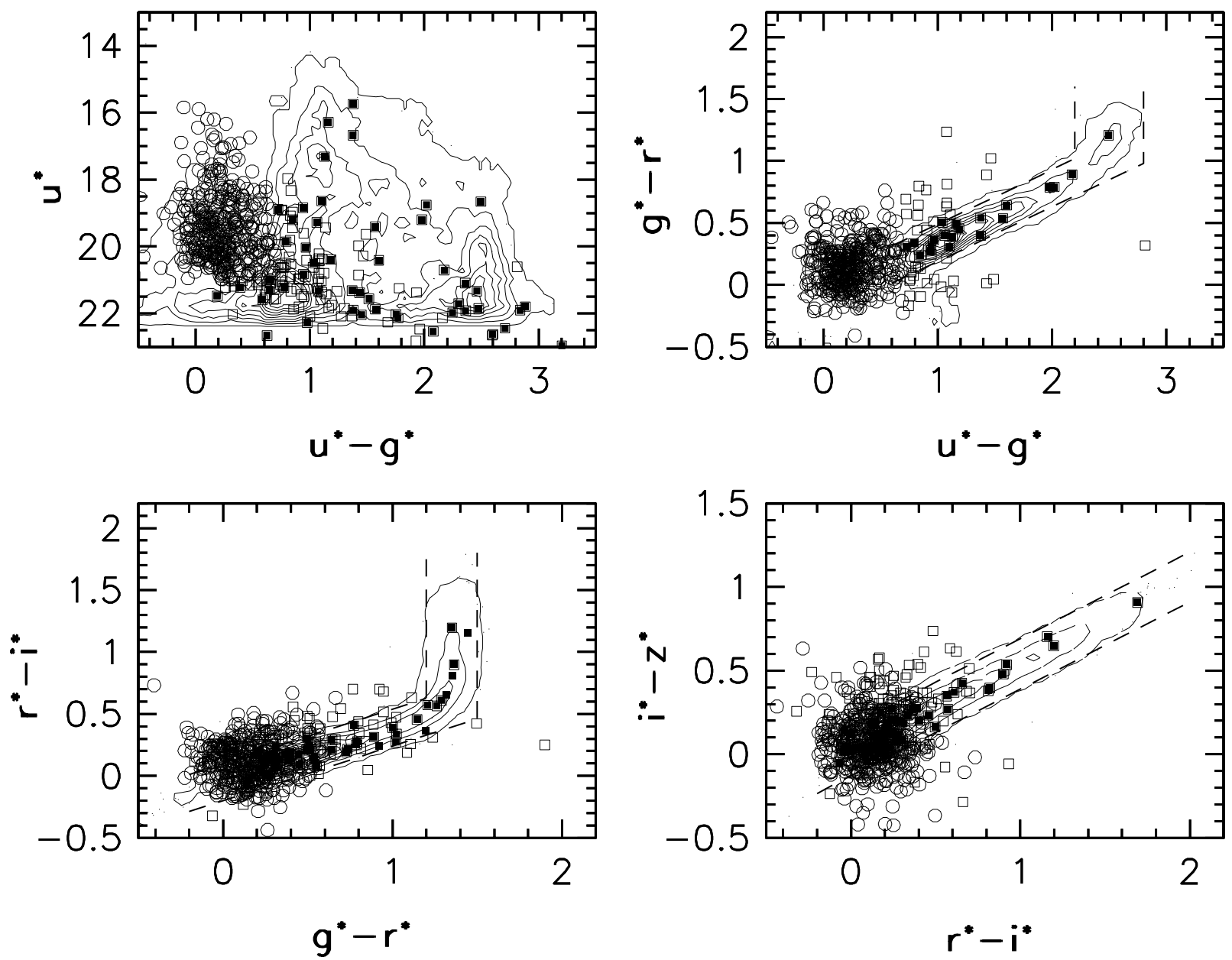

FIG. 14. - The $u^{*}$ vs. $u^{*}-g^{*}$ color-magnitude diagram and three color-color diagrams for stars (contours) and for optically unresolved radio sources brighter than $r^{*}=20.5$ (symbols). Open circles mark the 383 radio sources with UV excess satisfying $u^{*}<21$ and $u^{*}-g^{*}<0.7$. Open squares mark the 86 radio sources with nonstellar colors but without the UV excess, and the filled squares mark the 68 radio sources with colors indistinguishable from stellar. The dashed lines outline the boundaries of the four-dimensional stellar locus used to select sources with stellar colors. 
stars and for the 537 optically unresolved radio sources brighter than $r^{*}=20.5$ from the EDR sample. Of these, 383 sources have UV excess $\left(u^{*}<21\right.$ and $\left.u^{*}-g^{*}<0.7\right), 86$ sources have nonstellar colors, but without UV excess, and 68 sources have colors indistinguishable from stellar.

The surface density of stars to a magnitude limit of $r^{*}<20.5$ is more than 100 times higher than the surface density of quasars, and consequently the random associations are dominated by stars. The probability of finding an unresolved SDSS source with $r^{*}<20.5$ within 1".5 from a random position is $9 \times 10^{-4}$ (for the high Galactic latitudes discussed here, see K02). Given the number of FIRST sources $(107,654)$, the expected number of random associations is 97 , implying that $206(=303-97)$ objects $(11 \%$ out of $1900-97=1803$ objects) are true optical-radio associations with stellar colors. These results imply that the completeness of the SDSS quasar spectroscopic survey is at least $\sim 89 \%$. This fraction could be an underestimate if some of the associated sources with stellar colors are indeed stars with radio emission, as seems to be the case.

Optically unresolved objects with stellar colors are targeted by the SDSS spectroscopic survey if they are associated with FIRST objects (Richards et al. 2002; most of the quasars discussed here were not targeted using the final version of that algorithm; for more details see EDR). However, since the fraction of quasars with $i^{*}<19$ that are detected by FIRST is only $\sim 8 \%$ ( 0.94 vs. $\left.12.0 \mathrm{deg}^{-2}\right)$, the addition of these objects to the target list adds only $\sim 1 \%$ to the completeness of the spectroscopic sample. Nevertheless, these spectra are extremely useful for examining the nature of targeted sources and thus for testing the above conclusions. We visually inspected 155 available spectra from the sample of 303 objects discussed above, and classified them into 93 stars, six galaxies (compact, as determined from the imaging data), and 56 quasars. Some of these quasars have very unusual spectra; a few examples of quasars that were targeted only because they are FIRST sources are shown in Figure 15 . The low fraction of quasars (36\%) indicates that the fraction of quasars missed by the SDSS quasar spectroscopic survey due to their stellar colors may be as low as 5\% (except for redshift range of 2.5-3, where quasar colors mimic A stars in the SDSS system; see Richards et al. 2001). The fraction of spectroscopically confirmed stars $(60 \%)$ is about twice as high as the expected random association rate, implying that some of these are radio stars, in agreement with K02.

\subsubsection{The Color Difference between Radio-loud and Radio-quiet Quasars}

We now compare the colors of FIRST-detected quasars with those of quasars in general. Richards et al. (2001) noted that a FIRST-detected subsample of SDSS quasars has a larger fraction of intrinsically reddened sources than all SDSS quasars. Here we extend their analysis to a much larger sample. We study the $g^{*}-i^{*}$ color distribution because it maximizes the wavelength baseline, while avoiding the $u^{*}$ and $z^{*}$ bands, which are less sensitive than the other three bands. In our analysis we use the quasar redshifts produced by the SDSS spectroscopic pipelines; tests have shown that these redshifts are correct for approximately $97 \%$ of the objects (Schneider et al. 2002).

Richards et al. (2001) demonstrated that there is a tight correlation between the redshift and SDSS colors of qua-

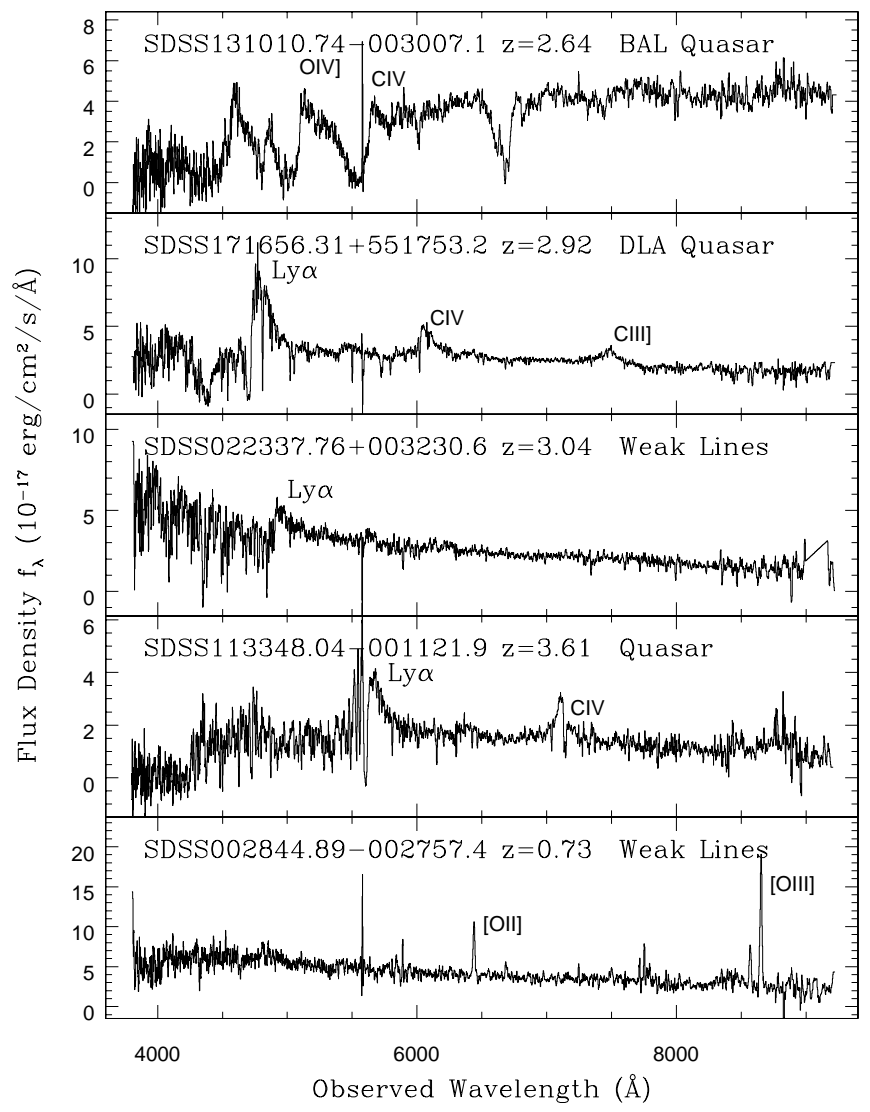

FIG. 15.-Examples of SDSS spectra (spectral resolution 2000) for optically unresolved sources with stellar colors and FIRST detections within 1".5. The object's name, redshift estimate, and classification is marked in each panel.

sars; this relation is clearly seen in the top panel of Figure 16. The distribution of 6567 optically selected and spectroscopically confirmed quasars with $i^{*}<18.5$ is shown by contours; those that are resolved (2095) are marked by crosses (mostly found at low redshift). The 280 FIRSTdetected quasars with $R_{i}>1$ (radio-loud ${ }^{39}$ ) are shown as filled circles, and the 161 FIRST-detected quasars with $R_{i}<1$ (radio-quiet) are shown as open circles. It is evident that the quasars colors vary with redshift, as discussed in detail by Richards et al. (2001). The thick solid line shows the median $g^{*}-i^{*}$ color of all optically selected quasars in the redshift range $1-2$. We subtract this median from the $g^{*}-i^{*}$ color to obtain a differential color, hereafter called color excess. The bottom panel shows the distribution of this color excess for 2265 quasars in that redshift range by filled squares (without error bars) and for 102 radio-loud quasars by circles.

The $g^{*}-i^{*}$ color-excess distribution for radio-loud quasars appears to be different from the distribution for the whole sample. First, the mean excess for the radio-loud subsample is redder by $0.09 \pm 0.02 \mathrm{mag}$, and the median excess by $0.08 \pm 0.02 \mathrm{mag}$. Second, the fraction of objects with very large color excess $(>0.4)$ is larger for the radioloud subsample; we find that $4.3 \% \pm 0.4 \%$ of quasars have such extreme $g^{*}-i^{*}$ colors, while this fraction is

\footnotetext{
${ }^{39}$ A detailed discussion of the radio loudness is presented in $\S 4.2$.
} 

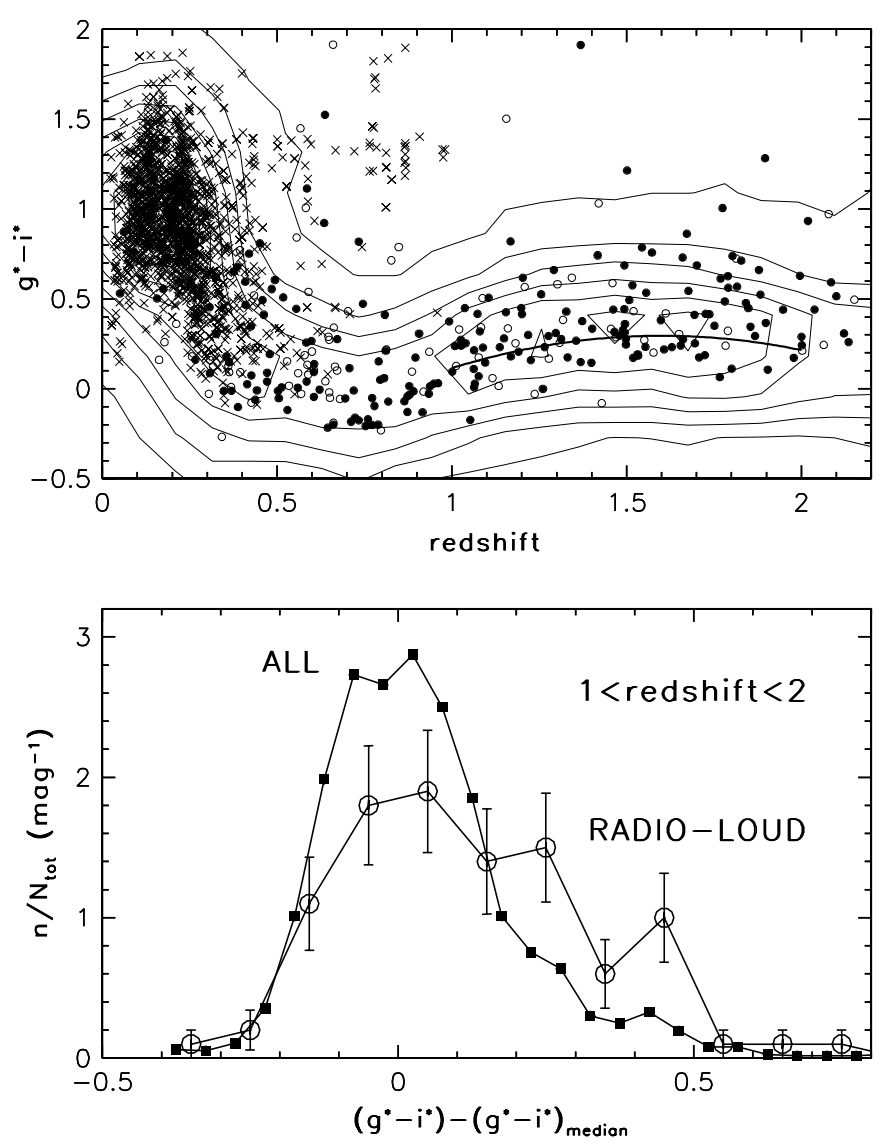

FIG. 16.-Top: Dependence of quasar $g^{*}-i^{*}$ color on redshift. The distribution of 6567 optically selected quasars with $i^{*}<18.5$ is shown by contours; those that are resolved (2095) are marked by crosses. The 280 FIRST-detected quasars with $R_{i}>1$ (radio-loud) are shown as filled circles, and the 161 FIRST-detected quasars with $R_{i}<1$ (radio-quiet) are shown as open circles. The thick solid line shows the median $g^{*}-i^{*}$ color of all optically selected quasars in the redshift range 1-2, which is subtracted from the $g^{*}-i^{*}$ color to obtain a color excess. Bottom: Distribution of the $g^{*}-i^{*}$ color excess for 2265 quasars in that redshift range by filled squares (without error bars) and for 102 radio-loud quasars by circles.

$14 \% \pm 4 \%$ for the radio-loud quasars. Equivalently, the fraction of radio sources in the subsample of quasars with extreme $g^{*}-i^{*}$ color excess $(\sim 20 \%)$ is 2.5 times higher than the corresponding overall fraction for radioloud quasars. The inspection of other color-color diagrams shows that the objects with extreme $g^{*}-i^{*}$ color-excess are shifted along the stellar locus in the $r^{*}-i^{*}$ versus $g^{*}-r^{*}$ color-color diagram. However, in the $g^{*}-r^{*}$ versus $u^{*}-g^{*}$ diagram they are shifted above the stellar locus and thus are easily distinguishable from stars (i.e., they are not missed by the SDSS quasar targeting pipeline). In the redder bands they are not such extreme outliers as in the bluer bands, as noted by Richards et al. (2001). Note that the wavelength dependence of this effect is qualitatively consistent with a reddening due to dust extinction (though, of course, it may have other causes).

The conclusion, that radio quasars have statistically different optical colors, is in agreement with an analogous difference in the distribution of spectral slopes determined from SDSS spectra. We compute spectral indices, $\alpha$, defined by $F_{\nu} \propto \nu^{\alpha}$, as described in Vanden Berk et al. (2002). Figure 17 compares the $\alpha$ distributions for 557 radio-loud quasars and for 6868 quasars without FIRST detections

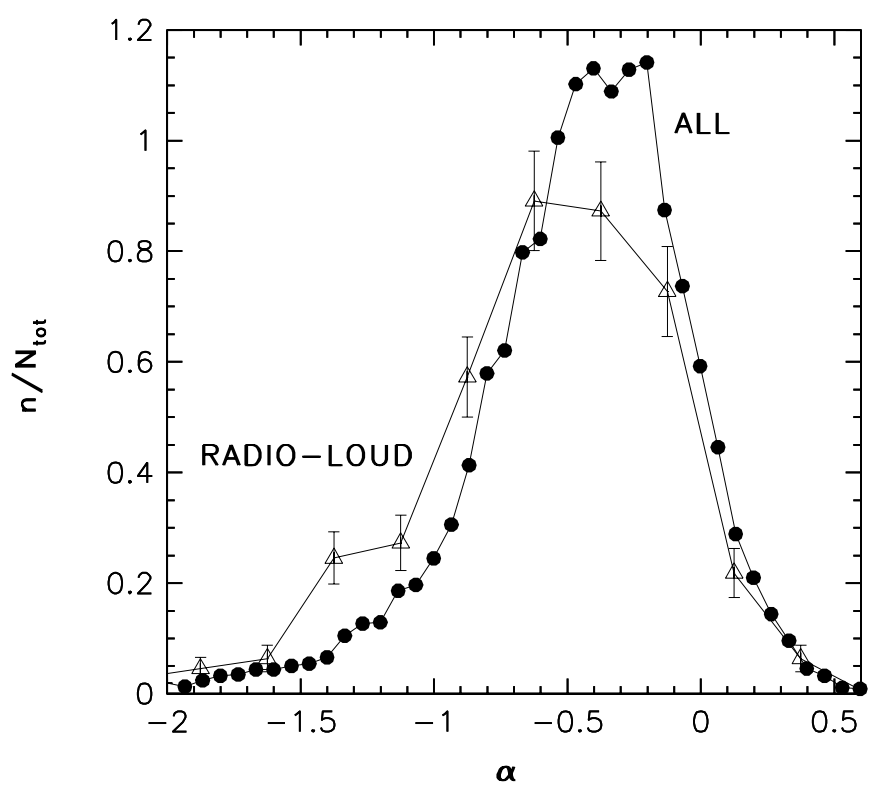

FIG. 17.-Optical spectral indices $\alpha\left(F_{\nu} \propto \nu^{\alpha}\right)$ determined from SDSS spectra for 6868 quasars that are brighter than $i^{*}=19$ (filled circles). The $\alpha$ distribution for a subsample of 440 radio-loud quasars (triangles with error bars) is skewed toward more negative values (redder optical spectra).

that are brighter than $i^{*}=19$. As is evident, the $\alpha$ distribution for radio-loud quasars is skewed toward more negative values (redder spectra). The mean and median of the distribution for radio-loud quasars are -0.59 and -0.52 , while they are -0.45 and -0.41 for the full sample (the accuracy of these estimates is $\sim 0.02$ ). Furthermore, the fraction of radio-loud quasars with $\alpha<-1$ is $18 \%$, while the corresponding fraction for the full sample is $8.4 \%$. We find no correlation between optical and optical-to-radio spectral indices for radio-loud quasars.

\subsubsection{The Optical Counts of Radio-loud and Radio-quiet Quasars}

Figure 18 shows the differential counts for optically unresolved and spectroscopically confirmed SDSS quasars from a $1030 \mathrm{deg}^{2}$ region. The turnover at $i^{*} \sim 19$ is due to the flux limit for spectroscopic targeting. The best fit to these counts in the $15.5<i^{*}<18.0$ range is

$$
\log (n)=-15.15+0.87 i^{*},
$$

where the counts are expressed per unit magnitude and per square degree. For illustration, an approximate estimate of the quasar counts to $i^{*} \sim 20$ is obtained by photometric selection of unresolved SDSS sources brighter than $u^{*}=21$ that show UV excess (for clarity, displayed only for $i^{*}>17.5$ ). These counts turn over for $i^{*} \gtrsim 20$ because of the $u^{*}<21$ selection cutoff. ${ }^{40}$

The optical counts of FIRST-detected quasars are affected by the $1 \mathrm{mJy}$ radio-flux cutoff. This effect can be removed by imposing a sufficiently large requirement on the value of the radio-to-optical flux ratio, so that the radio flux cutoff becomes inconsequential. We adopt a condition $R_{i}>1$, which selects 969 radio-loud quasars. Their optical

${ }^{40}$ The quasar counts from deep optical surveys do flatten for $i^{*} \gtrsim 20$, and the slope becomes $\sim 0.3$ for $i^{*} \lesssim 22$ (Pei 1995). 


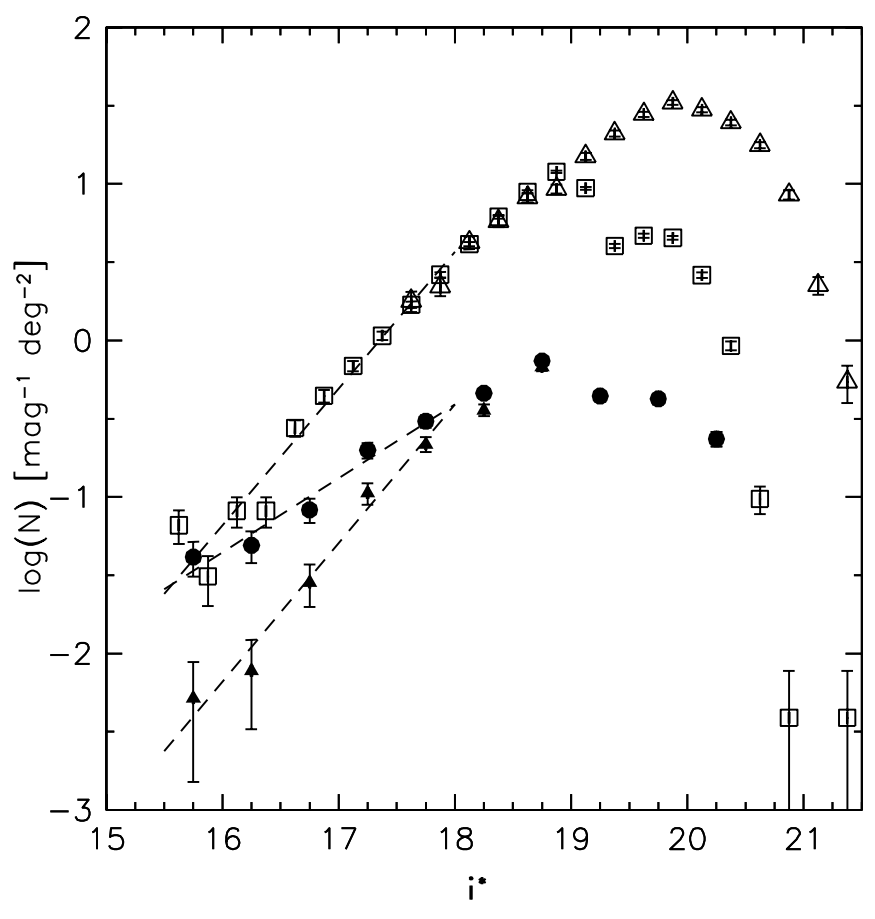

FIG. 18.-Differential counts for optically unresolved and spectroscopically confirmed SDSS quasars (open squares). The turnover at $i^{*} \sim 19$ is a selection effect due to a flux limit for spectroscopic targeting, as indicated by the open triangles that show the counts of UVX-selected optically unresolved SDSS sources brighter than $u^{*}=21$ (displayed only for $i^{*}>17.5$ for clarity). The counts for 1154 FIRST-detected optically unresolved objects are shown by filled circles. The filled triangles show counts for a subset of 969 FIRST-detected radio-loud sources with $R_{i}>1$. The dashed lines are the best linear fits in the $15.5<i^{*}<18.0$ range described in the text. For $i^{*}<18.5$ the fraction of FIRST-detected quasars is $13 \%$, and the fraction of radio-loud quasars is $\sim 8 \%$.

counts are shown in Figure 18; the best fit in the $15.5<i^{*}<18.0$ range is

$$
\log (n)=-16.35+0.89 i^{*} .
$$

The number counts versus magnitude slopes given by equations (11) and (12) are measured with an accuracy of $\sim 0.03$, and thus they are statistically identical; i.e., the fraction of radio-loud quasars is not a function of optical magnitude. The SDSS and FIRST data show that the fraction of quasars with $i^{*}<18.5$ that is radio-loud is $8 \% \pm 1 \%$ (the SDSS-FIRST sample includes 441 spectroscopically confirmed quasars with $i^{*}<18.5$ in $774 \mathrm{deg}^{2}$ of sky, and 280 of those have $R_{i}>1$; the control sample includes 4472 spectroscopically confirmed quasars with $i^{*}<18.5$ in $1030 \mathrm{deg}^{2}$ of sky).

Without a restriction on the radio-to-optical flux ratio, the optical counts of FIRST-detected quasars have a flatter slope because, at the bright optical $\mathrm{m}$ agnitudes, the FIRST survey also detects radio-quiet quasars. The counts of all 1154 FIRST-detected quasars from a $1230 \mathrm{deg}^{2}$ region are shown in Figure 18 as filled circles, and the best fit is

$$
\log (n)=-8.91+0.47 i^{*} \text {. }
$$

This result is in agreement with the optical counts of quasars discovered by the FIRST Bright Quasar Survey (FBQS, White et al. 2000). For $i^{*}<18.5$ the cumulative fraction of FIRST-detected quasars is $13 \%$.

\subsection{The Quasar Radio Dichotomy}

There is controversy in the literature about the existence of a bimodality in the distribution of radio loudness ${ }^{41}$ of quasars. Strittmatter et al. (1980) pointed out that the radioto-optical flux density ratio for optically selected quasars appears bimodal. Many other studies found similar results (e.g., Kellermann et al. 1989; Miller, Peacock, \& Mead 1990; Stocke et al. 1992; Hooper et al. 1995; Serjeant et al. 1998, and references therein). However, some authors question the existence of this so-called "radio dichotomy," e.g., Condon et al. (1981) and White et al. (2000). Most of these studies are based on small samples, which typically include only 100 sources, except for the FBQS (White et al. 2000) with 600 objects.

\subsubsection{The Distribution of SDSS-FIRST Quasars in the Optical-Radio Flux Plane}

In this subsection we determine the unbiased distribution of the radio-to-optical flux ratio, $R_{i}$ and argue that it suggests the existence of a radio dichotomy. Following White et al. (2000), we compute $R_{i}$ with observed (i.e., no $K$-correc$\operatorname{tion}^{42}$ ) $t$ and $i^{*}$ magnitudes (eq. [5).] To ensure that the optical and radio fluxes are reliable, we constrain the sample to optically unresolved sources with $i^{*}<21$ and $t<16.5$, resulting in 3066 objects. Their distribution in the $t$ versus $i^{*}$ diagram is shown in the top panel in Figure 19.

If the distribution of the radio-to-optical flux ratio, $R_{i}$, is not a function of the optical luminosity or redshift, then the distribution of $R_{i}$ should be uncorrelated with the apparent optical and radio magnitudes. However, the optical and radio flux limits $\left(i^{*}<21\right.$ and $\left.t<16.5\right)$ have a significant effect on the observed $R_{i}$ distribution and must be taken into account properly. ${ }^{43}$ The solid and four dashed lines in Figure 19 extending from the upper left to the lower right corner show five characteristic values of $R_{i}$. The three dotdashed lines, perpendicular to the $R_{i}=$ const. lines, define two strips in the $t-i^{*}$ plane that are not affected by the optical and radio flux limits for sources with $0<R_{i}<4$. The $R_{i}$ distributions for 670 sources from these strips are shown as the filled symbols in the bottom panel in Figure 19. The two histograms are statistically the same (for clarity, Poisson error bars are shown only for one histogram), suggesting that the distribution of the radio-to-optical flux ratio for quasars is independent of apparent optical and radio magnitudes.

The $R_{i}$ distributions show a local maximum at $R_{i} \sim 2.8$. Given that the majority of SDSS quasars $(\sim 90 \%)$ with $i^{*}<18.5$ are not detected by FIRST, the unobserved part of the $R_{i}$ distribution must eventually rise for $R_{i}<0$. In

\footnotetext{
${ }^{41}$ Two definitions of radio loudness are found in the literature. Here we use the radio-to-optical flux ratio to quantify radio loudness; the alternative approach based on radio luminosity is discussed in Appendix C.

${ }^{42}$ There is no difference between corrected and uncorrected $R_{i}$ as long as the optical and radio spectral slopes are the same, as is often assumed $\left(\alpha_{\text {opt }}=\alpha_{\text {radio }}=-0.5\right)$.

${ }^{43}$ For example, consider a uniform distribution of points in the $x-y$ plane that is sampled in a square defined by $0<x<1$ and $0<y<1$. The sampled distribution of variable $\phi=y-x$ has a local maximum for $\phi=0$, although the underlying distribution is uniform. The unbiased $\phi$ distribution in the $-0.5<\phi<0.5$ range can be easily determined by considering only the square defined by $y=x+0.5, y=x-0.5, y=-x+0.5$, and $y=-x+1.5$. It is possible to account for the selection effects using the whole sample, as described by, e.g., Petrosian (2001).
} 

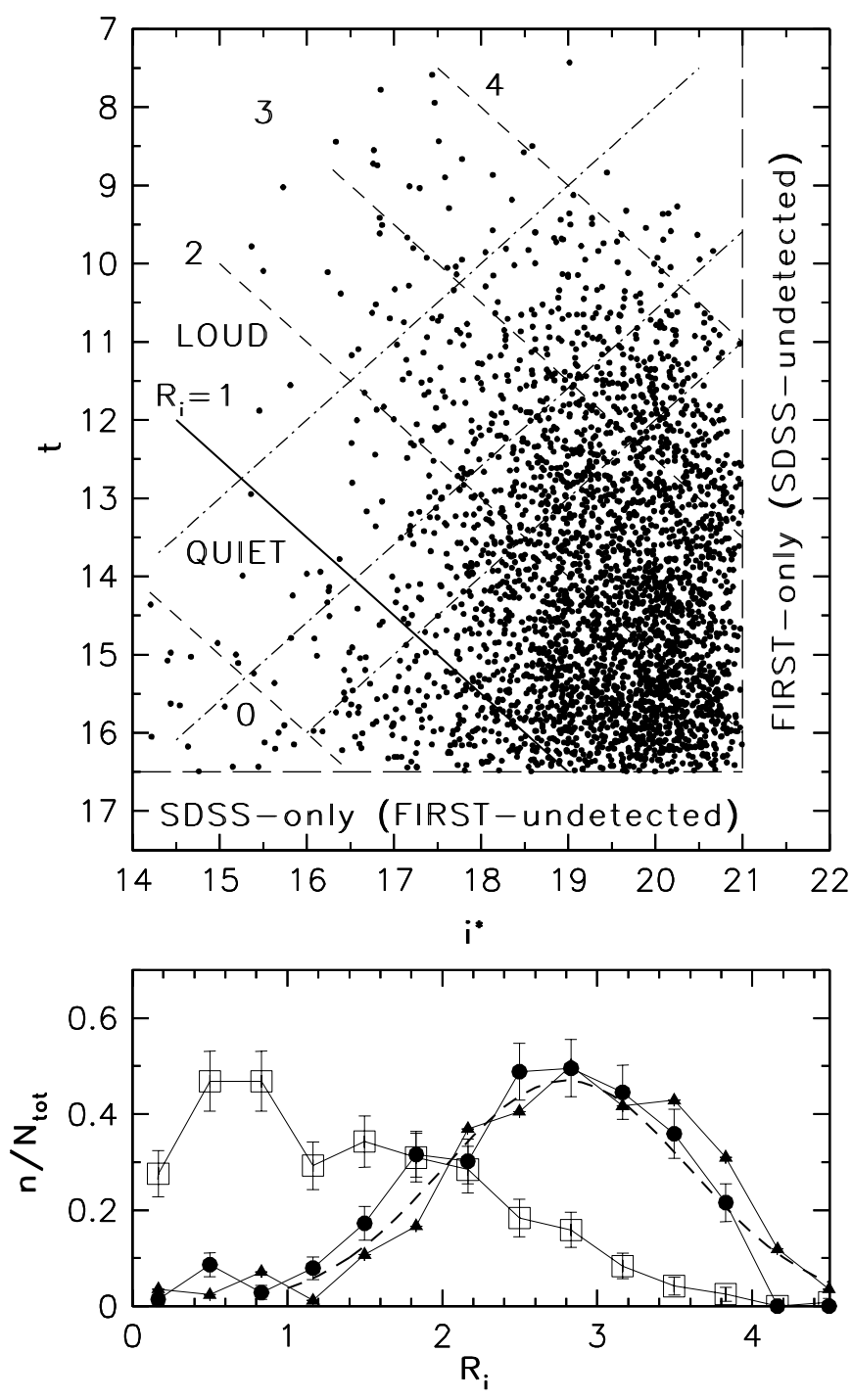

FIG. 19.-Top: The $t$ (radio magnitude) vs. $i^{*}$ (optical magnitude) distribution of the 3066 optically unresolved SDSS sources detected by FIRST with $i^{*}<21$ and $t<16.5$. The diagonal solid line shows the traditional radio-loud/quiet division line $\left(R_{i}=1.0\right)$, and the four short-dashed lines show $R_{i}=0,2,3$, and 4 , as marked. In the bottom panel the histogram marked by open squares shows the $R_{i}$ distribution for 359 sources with $i^{*}<18$. The two histograms marked by filled circles and triangles show the $R_{i}$ distribution for the 670 sources selected from the two strips defined by the three dot-dashed lines shown in the top panel. The dashed line is a best Gaussian fit to the sum of these two histograms for $R_{i}>1$.

principle, the unobserved part of the $R_{i}$ distribution could be a monotonically decreasing tail extending far into negative values. However, deep radio studies of smaller samples (e.g., Kellermann et al. 1989 at $6 \mathrm{~cm}$ ) indicate that nearly all optically bright radio-quiet sources have at most a factor $\sim 1000$ weaker radio emission than radio-loud sources $\left(-2 \lesssim R_{i} \lesssim 0\right)$. This implies a local minimum in the $R_{i}$ distribution, which appears to be in the region $0 \lesssim R_{i} \lesssim 1$ (see Appendix D for a discussion of the 6-to- $20 \mathrm{~cm}$ spectral index distribution for quasars). Thus the $R_{i}$ distribution observed for SDSS-FIRST quasars, in particular its rise between $R_{i} \sim 1$ and $R_{i} \sim 3$, suggests the existence of the radio dichotomy. ${ }^{44}$ The loud-quiet division line at $R_{i} \sim 1$ is

${ }^{44}$ We do not address a possibility pointed out by the referee that the weak radio emission from radio-quiet quasars may be dominated by starburst emission from quasar host galaxies. consistent with previous work (e.g., Urry \& Padovani 1995) and implies that every quasar detected by FIRST and fainter than $i^{*}=18.5$ is radio-loud. An unbiased estimate of the number ratio of radio-loud to radio-quiet quasars is not possible with the available data (our estimate that $8 \% \pm 1 \%$ of quasars are radio-loud is valid for a sample limited by optical flux, $\left.i^{*}<18.5\right)$. Such an estimate could be determined if, for example, the data were available in the strip bounded by lines at $t=34-i^{*}$ and $t=35-i^{*}$; that is, if radio observations of SDSS quasars that are deeper than the FIRST survey were available for a sufficiently large number of sources.

The local minimum in the $R_{i}$ distribution is in conflict with the suggestion by White et al. (2000) that the bimodal distribution of the radio properties may be spurious. However, they did not correct the observed $R_{i}$ distribution for selection effects. The FBQS was limited in the optical range by the POSS-I $E$ magnitude cutoff $E<17.8$ (corresponding to $i^{*} \lesssim 18$ ) and in the radio by the FIRST sensitivity limit $(t<16.4)$. Since the quasar counts steeply increase with optical apparent magnitude, the sample is dominated by sources near the flux limit, and the $R$ distribution is heavily weighted by sources with $R_{i}$ close to $R_{\text {cutoff }}=0.4\left(i_{\text {cutoff }}-t_{\text {cutoff }}\right) \sim 0.6$. Thus the fraction of radio-intermediate quasars detected by the FBQS is larger than in other surveys. To illustrate this effect, we follow White et al. and simply determine the $R_{i}$ distribution for all objects in our sample with $i^{*}<18$ (359), shown by open squares in the bottom panel in Figure 19. The counts rise toward small $R_{i}$ without strong indication for a local minimum, because the sample is biased by objects around the faint cutoff. ${ }^{45}$

The distribution of data points in the top panel in Figure 19 illustrates why the quasar samples detected in older bright radio and optical surveys had very different $R_{i}$ distributions. For example, in a radio survey with a faint flux limit of $0.1 \mathrm{Jy}(t=11.4$, or $100 \mathrm{mJy})$ nearly all sources have $R_{i}>2$ (i.e., those above the $t=11.4$ line). On the other hand, quasars detected in a bright optical survey sensitive to $i^{*}=16.5$ (i.e., those to the left from the $i^{*}=16.5$ line) separate into two types: those with $R_{i} \gtrsim 2$ and those with $R_{i} \lesssim 1$. The more sensitive SDSS and FIRST data sample a much larger portion of the $t-i^{*}$ plane and provide an improved estimate of the overall $R_{i}$ distribution.

In order to rule out the effects of high spatial resolution on FIRST flux measurements, in Figure 20 we reproduce Figure 19 using NVSS instead of FIRST. As is evident, the $R_{i}$ distribution remains practically unchanged.

\subsubsection{The Radio-Loudness as a Function of Luminosity and Redshift}

Hooper et al. (1995) argued that the fraction of radioloud objects is a function of both optical luminosity and redshift. The sample discussed here is sufficiently large to test this suggestion. The top panel in Figure 21 compares the distribution of 280 radio-loud quasars with $i^{*}<18.5$ and $R_{i}>1$ to the distribution of 4472 optically selected quasars

${ }^{45}$ This bias may be responsible for the increased fraction of broad absorption-line quasars with intermediate radio-to-optical flux ratios $\left(0<R_{i}<1\right)$ discussed by Menou et al. (2001). However, their sample is too small to confidently exclude the possibility that quasars with intermediate radio-to-optical flux ratios are more likely to have broad absorption lines. 

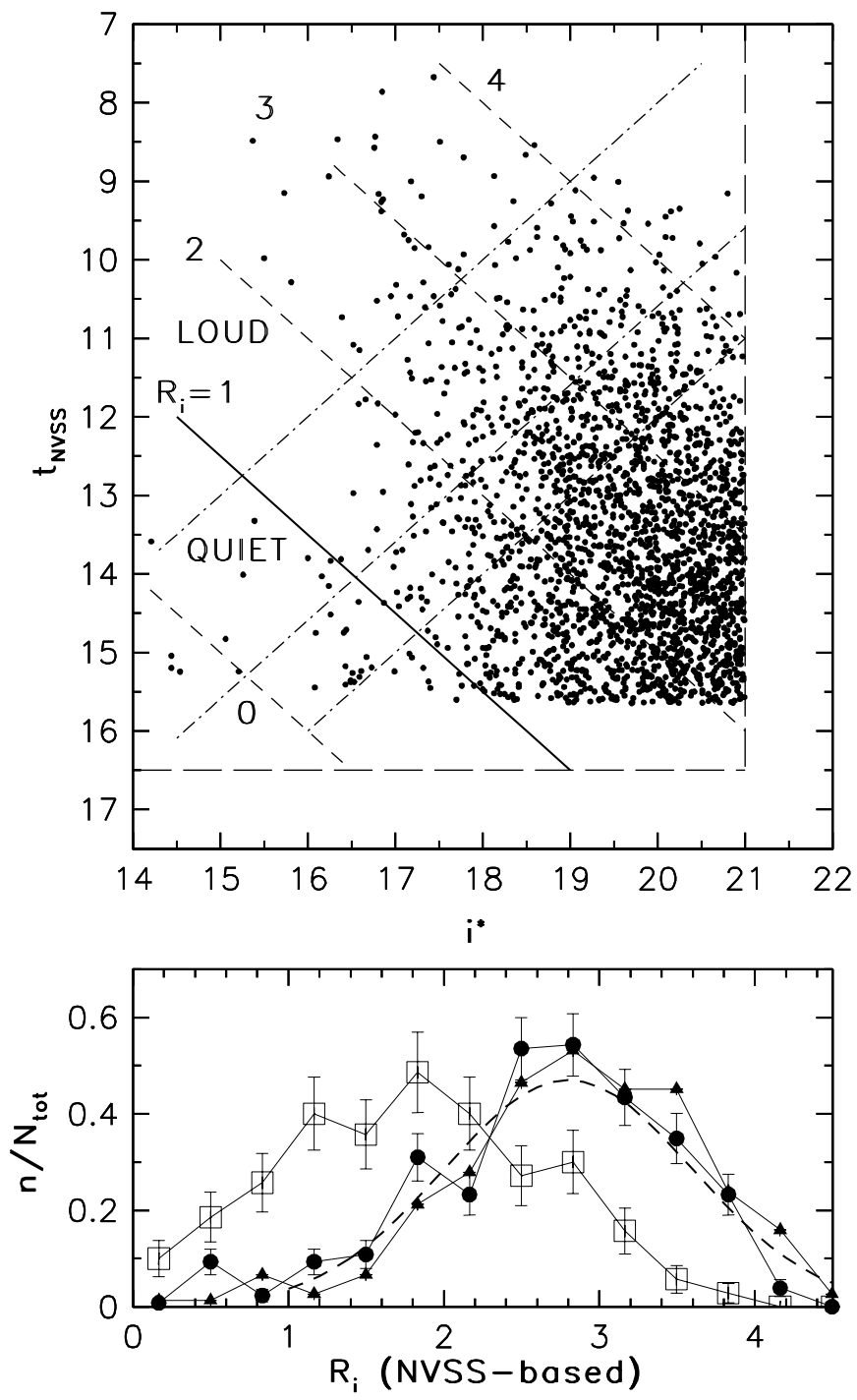

FIG. 20.- Same as Fig. 19, except that NVSS is used instead of FIRST. Note that the $R_{i}$ distributions shown by filled symbols are practically the same as in Fig. 19.

brighter than $i^{*}=18.5$ in the absolute magnitude, $M_{i}$, versus redshift plane. The absolute magnitude is strongly correlated with redshift because of the faint optical cutoff and steeply rising optical counts.

The middle panel compares the absolute magnitude histogram for the whole sample and for the radio-loud subsample. The bottom panel compares the redshift histograms. There are no significant differences between the distributions of $M_{i}$ and redshift for the radio subsample and for the whole sample. The small differences for $M_{i} \sim-23$ and for redshift $\sim 0.5$ are due to a clump of about 10 objects and are significant only at the $1-2 \sigma$ level.

We conclude that our sample does not support the suggestion that the fraction of radio-loud quasars depends on optical luminosity or redshift. However, we caution that, at least in principle, the luminosity and redshift dependence could conspire to produce no observed effect (e.g., if the radio-loud fraction decreases with redshift and increases with luminosity) as a result of the strong correlation between luminosity and redshift in a magnitude-limited sample.
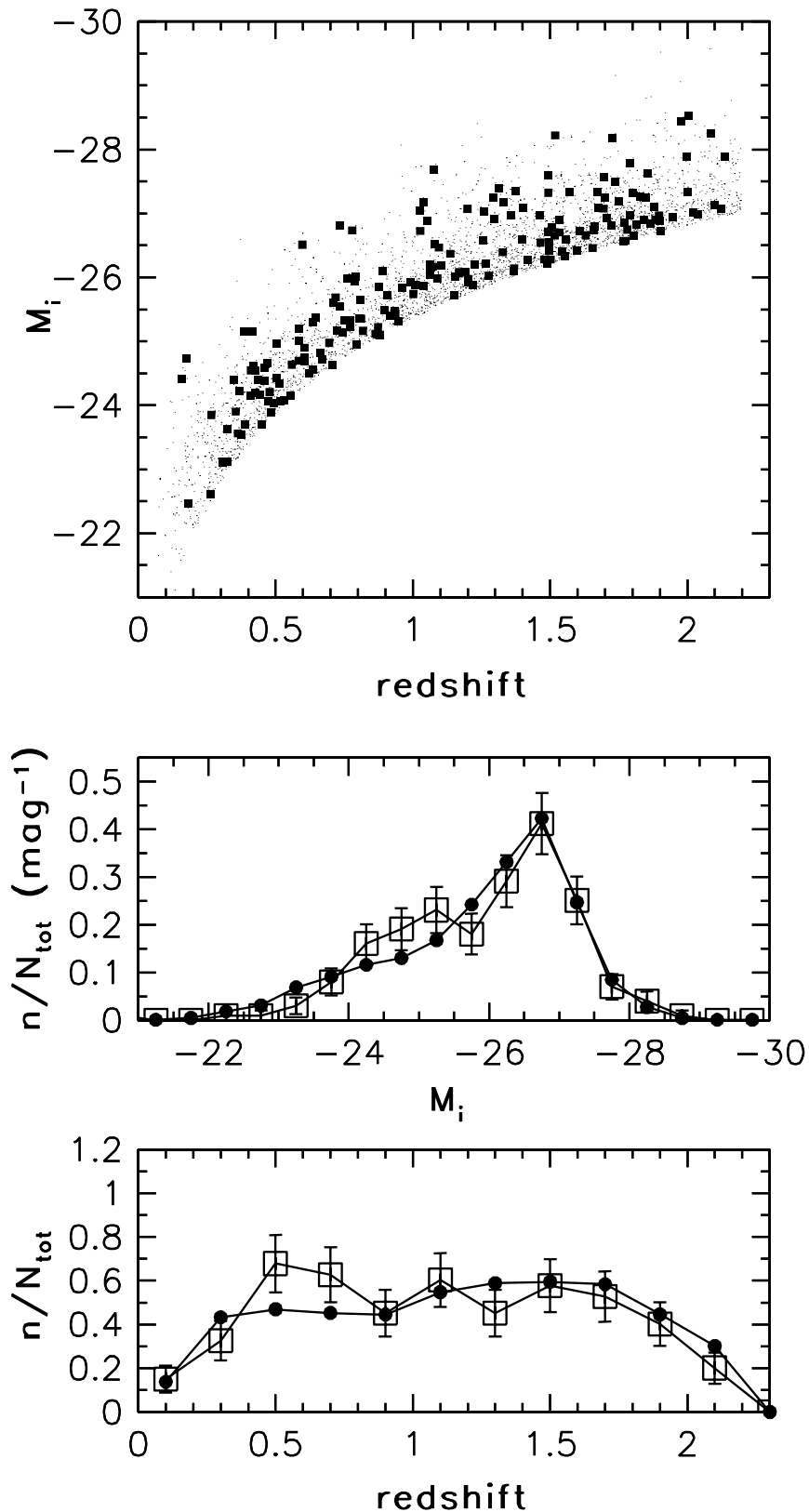

FIG. 21.-Top: Redshift and absolute magnitude distribution $\left(M_{i}\right)$ for 4472 optically selected quasars with $i^{*}<18.5$ (dots) and for a subsample of 280 radio-loud quasars $\left(R_{i}>1\right)$ (squares). Middle: Distribution of absolute magnitudes for all quasars (dots without error bars) and for the radio subsample (squares) from the same redshift range as shown in the top panel. The bottom panel shows the corresponding redshift distribution (same notation). There is no significant difference between the distributions for the radio subsample and for the whole sample.

\section{THE OPTICAL AND RADIO PROPERTIES OF SDSS-FIRST GALAXIES}

Having analyzed properties of optically unresolved objects with radio detections, we turn now to galaxies. In this section we analyze the galaxy distribution in the space spanned by optical and radio fluxes, optical colors, redshift, and emission-line properties. The analyzed sample includes 15,683 optically resolved sources brighter than $r^{*}=21$ detected by FIRST; for a subsample of 5454 sources SDSS spectra are available. We also use a control imaging sample 
of 304,147 optically resolved sources and a control spectroscopic sample that includes 141,920 galaxies.

First we compare the optical properties of radio galaxies with the properties of galaxies from the control samples. We find that they have different optical luminosity distributions, while their color distributions are indistinguishable in subsamples selected by optical luminosity and redshift. This analysis is fully based on directly observed magnitudes, without taking into account $K$-corrections. The effect of the optical $K$-correction in the relevant redshift range $(\lesssim 0.4)$ is to dim and redden galaxies by a few tenths of a magnitude (Blanton et al. 2001 and references therein).

SDSS spectra are used to compute the line strengths for several characteristic emission lines, which allow classification of galaxies into starburst galaxies and active galactic nuclei (AGNs). We find that the fraction of radio galaxies whose emission-line ratios indicate an AGN rather than a starburst origin $(30 \%)$ is 6 times larger than the corresponding fraction for all SDSS galaxies $\left(r^{*}<17.5\right)$. The radio emission from AGNs is more concentrated than radio emission from starburst galaxies, and the AGN-to-starburst galaxy number ratio decreases with radio flux.

\subsection{The Optical Properties of Radio Galaxies 5.1.1. The Morphological Properties}

First we examine the distribution of radio galaxies in the $r^{*}$ versus $g^{*}-r^{*}$ color-magnitude diagram (where morphological types are well separated; see $\S$ 2.1.2), and then we discuss the visual inspection of multicolor SDSS images. Figure 22 displays the $r^{*}$ versus $g^{*}-r^{*}$ color-magnitude for SDSS-FIRST galaxies and for all SDSS galaxies. The top panel shows 19,496 SDSS-FIRST galaxies brighter than $r^{*}=21.5$ (for illustration, in this figure we relax the condition that $r^{*}<21$ ), and the bottom panel shows 4300 galaxies for which SDSS spectra are available (the spectroscopic sample is practically complete for $r^{*} \lesssim 17.7$ in the regions of sky studied here). The dashed lines outline the regions discussed in $\S 3.1$ and Figure 2.

The color-magnitude distribution of radio galaxies is markedly different from that of SDSS galaxies as a whole; at the bright end $\left(r^{*}<17.5\right)$ the fraction of radio galaxies does not strongly depend on color (i.e., galaxy type, S01), while at the faint end it is strongly dependent on color. For example, in region IId the radio fraction is $\sim 30$ times larger than in region IIa, although both regions span the same $r^{*}$ magnitude range. Table 1 lists the surface densities of radio galaxies, expressed as a fraction of all galaxies, for each of the seven regions outlined in Figure 22.

Region IId includes the luminous red galaxies (LRGs), which are the reddest galaxies in the $r^{*}$ versus $g^{*}-r^{*}$ colormagnitude diagram, due to the effects of $K$-corrections in the $g$ and $r$ bands (E01). Despite being among the most distant SDSS galaxies (redshifts up to $\lesssim 0.55$ ), a strikingly large fraction of LRGs ( $3 \%$ in region IId; see Table 1$)$ have radio counterparts.

We examine next the optical color images of radio galaxies. The 4152 galaxies with $r^{*}<17.5$ are sufficiently bright and large for SDSS imaging to capture detailed morphological information. Following S01, we first divide them into 1153 blue galaxies with $u^{*}-r^{*}<2.22$ and 2999 red galaxies with $u^{*}-r^{*}>2.22$ (for bright galaxies the $u^{*}-r^{*}$ color-based separation is more robust than $g^{*}-r^{*}$ classification, S01). Visual inspection of 1084 multicolor $(g-r-i$
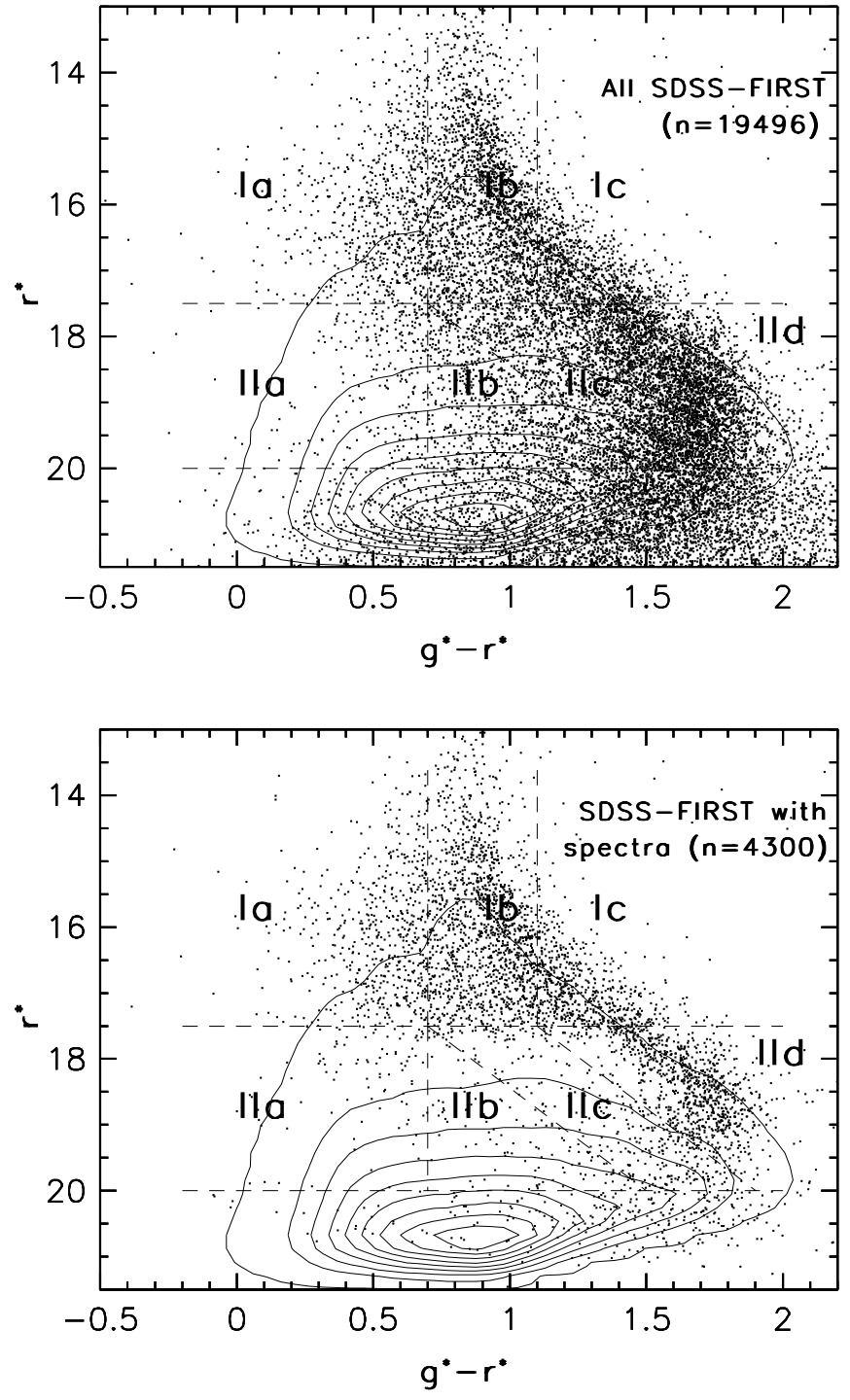

FIG. 22.-The $r^{*}$ vs. $g^{*}-r^{*}$ color-magnitude diagram for SDSS-FIRST galaxies (dots) compared with the distribution of all SDSS galaxies, shown by linearly spaced contours. Top: 19,496 SDSS-FIRST galaxies brighter than $r^{*}=21.5$. Bottom: 4300 galaxies for which SDSS spectra are available (the area covered by the latter subsample is $63 \%$ of the area covered by the former). The dashed lines outline regions with different galaxy morphology and fraction of radio galaxies, as listed in Table 1.

composites) SDSS images from the EDR subsample confirms that $\gtrsim 80 \%$ of the blue radio galaxies are spiral galaxies, while $\gtrsim 90 \%$ of the red radio galaxies are elliptical galaxies, in agreement with the results for the full sample (S01). We found only a few examples of clearly blue elliptical galaxies.

The visual inspection indicates that the incidence of merging galaxies and galaxies with disturbed structure among the blue radio galaxies is higher than for a random sample of blue galaxies. A detailed quantification of this effect will be presented in a future publication. For illustration, Figure 23 shows composite $1^{\prime} \times 1^{\prime} g-r-i$ images of 12 radio galaxies, selected to include interesting examples. The radio position is marked by a cross. The merging nature can be discerned more easily in Figure 24, which shows the same galaxies with a stretch chosen to emphasize the galactic 

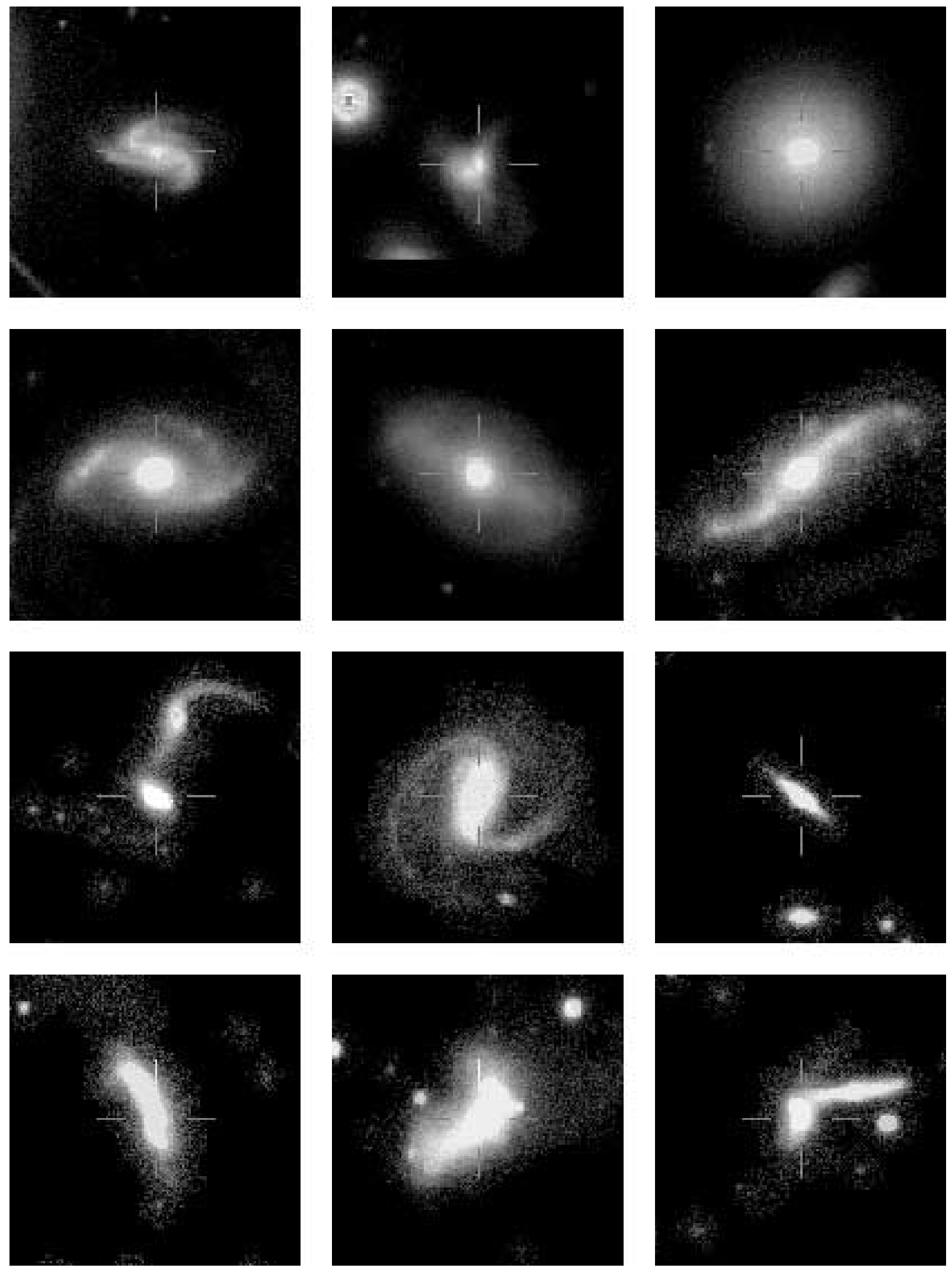

FIG. 23.-Mosaic of true-color $g-r-i$ color composite images for a subsample of SDSS-FIRST galaxies with $r^{*}<17.5$ and $u^{*}-r^{*}<2.22$. The image size is $1^{\prime} \times 1^{\prime}$, with east toward the top and north toward the right. The position of the associated FIRST source is marked by a cross. [See the electronic edition of the Journal for a color version of this figure.] 


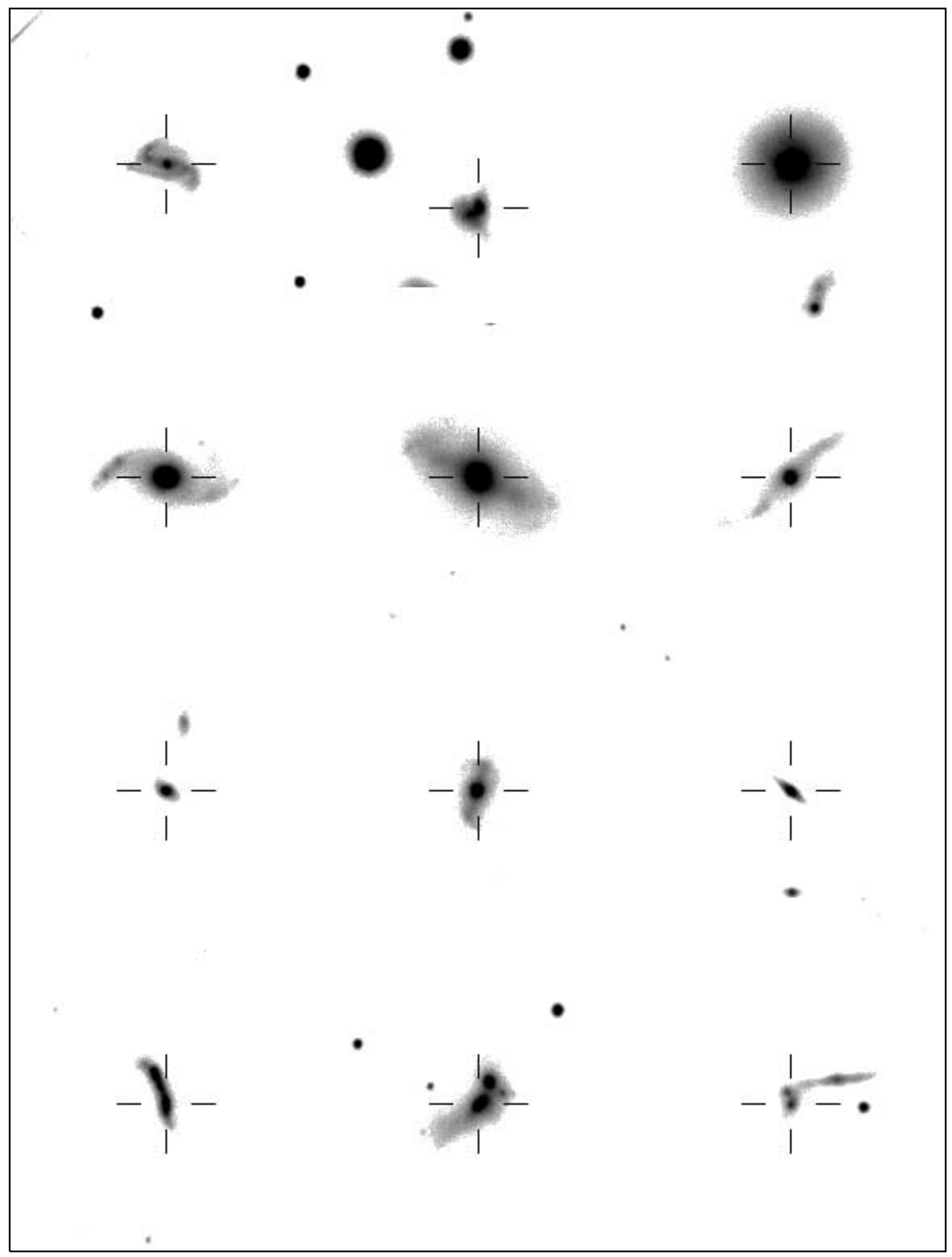

FIG. 24.-SDSS $r$-band images for the same galaxies as in previous figure. The stretch is chosen to emphasize galactic nuclei, and the display is negative. 
nuclei. Obvious examples of mergers are shown in the top and bottom center panels.

\subsubsection{The Optical Colors of Radio Galaxies}

We now compare the optical colors of radio galaxies and other galaxies. We demonstrate that optical colors of radio galaxies are similar to those of all galaxies selected by the same redshift and optical luminosity criteria, despite being markedly different in flux-limited samples.

The two top panels and the bottom left panel in Figure 25 compare the distributions of SDSS galaxies and 4152 radio galaxies brighter than $r^{*}=17.5$ in color-color diagrams. The radio galaxies appear to have redder color distributions than the full sample. This is a selection effect; in a fluxlimited sample radio galaxies tend to have larger redshifts than all galaxies as a whole (demonstrated further below). Because of larger redshifts and optical $K$-correction reddening (which we did not take into account), their observed colors are redder (the evolutionary effects may also contribute to this reddening). When selected from the same redshift range, the colors of radio galaxies are similar to those of other galaxies. This point is illustrated in Figure 25 (bottom right), where we compare the redshift versus $r^{*}-i^{*}$ color distributions for all galaxies with spectra; radio galaxies follow the same distribution as all SDSS galaxies with spectra.

The tendency that the colors of radio galaxies in a fluxlimited sample are biased toward the red end affects the classification based on observed $u^{*}-r^{*}$ color. The top left panel in Figure 26 compares the $r^{*}$ versus $u^{*}-r^{*}$ color-magnitude distributions for galaxies with $r^{*}<17.5$ (practically a complete sample). The fact that radio galaxies have redder $u^{*}-r^{*}$ color is more clearly visible in the top right panel, where we compare the $u^{*}-r^{*}$ histograms. The shapes of the two distributions are similar, with the radio galaxy color distribution redder by $\sim 0.3 \mathrm{mag}$. The vertical dot-dashed line at $u^{*}-r^{*}=2.22$ shows the classification boundary between blue and red galaxies at low redshifts (S01). Assuming that all radio galaxies are redder by $\sim 0.3 \mathrm{mag}$, the application of this condition to radio galaxies brighter than $r^{*}=17.5$ results in $\sim 20 \%$ of " blue " galaxies being misclassified as "red" galaxies. Since this effect is not very strong,
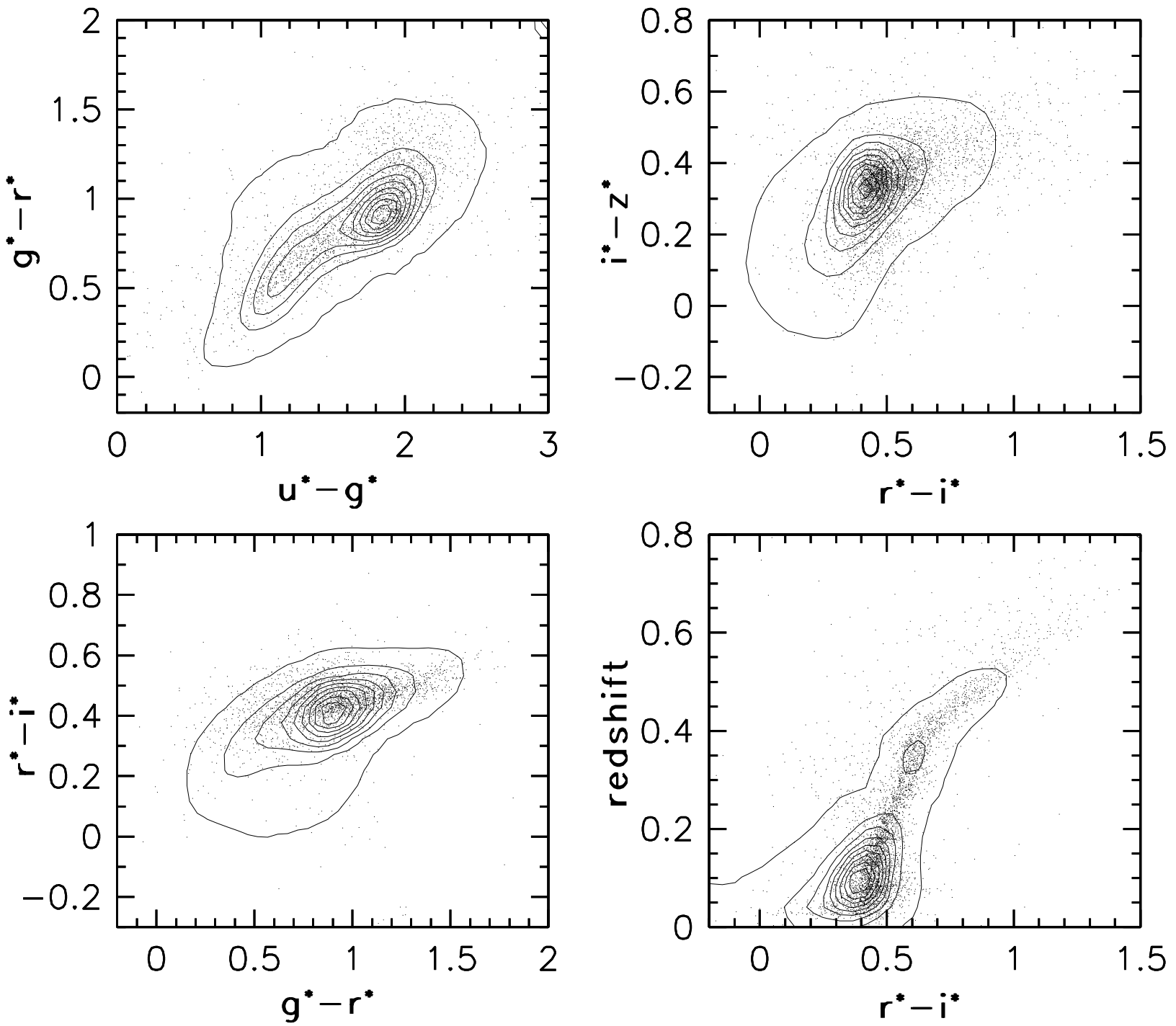

FIG. 25.-Optical color-color diagrams for 4152 SDSS-FIRST galaxies with $r^{*}<17.5$ (dots) compared with the distribution of all SDSS galaxies, shown by linearly spaced contours. Bottom right: Correlation between the redshift and the $r^{*}-i^{*}$ color for galaxies with spectra $(141,920$ for the full sample and 4300 for radio-galaxies). 

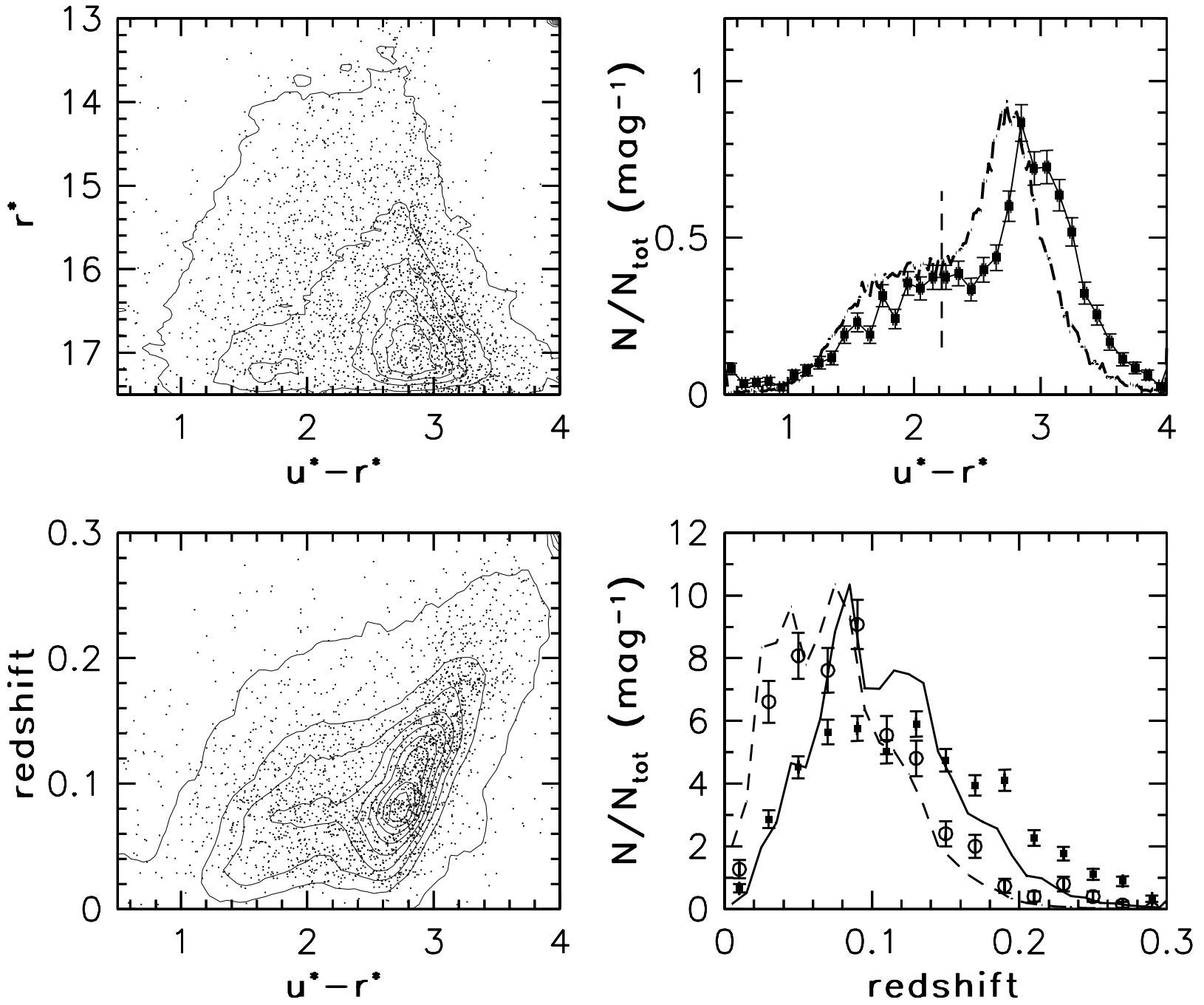

Fig. 26. - Top left: Distribution of the 91,422 galaxies (contours) with $r^{*}<17.5$ (contours) in the $r^{*}$ vs. $u^{*}-r^{*}$ color-magnitude diagram; a subsample of 2563 radio galaxies by dots. Top right: Arginal $u^{*}-r^{*}$ distributions for all (dashed line) and radio (symbols, solid line) galaxies; the latter are redder. Bottom left: Comparison of the distributions of radio galaxies and all galaxies in the $u^{*}-r^{*}$ color-redshift plane. Bottom right: Comparison of the redshift distributions of for two color-selected subsamples (dashed line, all; open circles, radio) for galaxies with $u^{*}-r^{*}<2.22$, and solid line (all) and filled squares (radio) for galaxies with $u^{*}-r^{*}>2.22$. Radio galaxies in a flux-limited sample are biased toward larger redshifts.

in the rest of this work we retain $u^{*}-r^{*}=2.22$ as the separation boundary between blue and red galaxies.

The $u^{*}-r^{*}$ color distribution of radio galaxies is somewhat redder than that of other galaxies because radio galaxies are sampled at higher redshifts. The bottom left panel in Figure 26 compares the redshift versus $u^{*}-r^{*}$ distributions for galaxies with $r^{*}<17.5$. They are the same ${ }^{46}$ to within $\sim 0.1 \mathrm{mag}$, further demonstrating that the intrinsic optical colors of radio galaxies are not very different from other galaxies. The remaining difference in colors of $\sim 0.1$ mag is due to the color-luminosity relation and differences in luminosity distributions between radio and other galaxies, which are discussed in the next section.

The redshift distributions for galaxies bluer and redder than $u^{*}-r^{*}=2.22$ and with $r^{*}<17.5$ are shown in the bottom right panel in Figure 26. The sharp features visible in these curves are not due to Poisson noise (the histograms are

${ }^{46}$ This statement is based on the comparison of $u^{*}-r^{*}$ histograms for narrow $(\sim 0.01-0.05)$ redshift slices in the range $0.05<$ redshift $<0.30$. based on a sample including $\sim 90,000$ galaxies); rather, they reflect the large-scale structure in the distribution of galaxies. The redshifts of radio galaxies tend to be larger than for other galaxies from the same flux-limited sample, and this effect is especially pronounced for red galaxies.

In summary, the broadband optical colors of radio galaxies in a flux-limited sample are redder than for all galaxies in the sample. This effect can be explained by the color-redshift relations; when confined to the same redshift range, the colors of radio galaxies are similar to those of other galaxies.

\subsubsection{The Optical Luminosity Distribution of Radio Galaxies}

We now compare the optical luminosity distribution of radio galaxies with that of all galaxies selected from the same narrow redshift range. The top left panel in Figure 27 shows the $r^{*}$ versus $u^{*}-r^{*}$ color-magnitude distribution for galaxies with $r^{*}<17.5$, redshift in the range $0.08-0.12$, and, for radio galaxies, $R_{r}>0.4$. The condition on $R_{r}$ ensures that the radio flux limit is not relevant (all galaxies with $R_{r}>0.4$ and $r^{*}<17.5$ are brighter than $t=16.5$; for a 

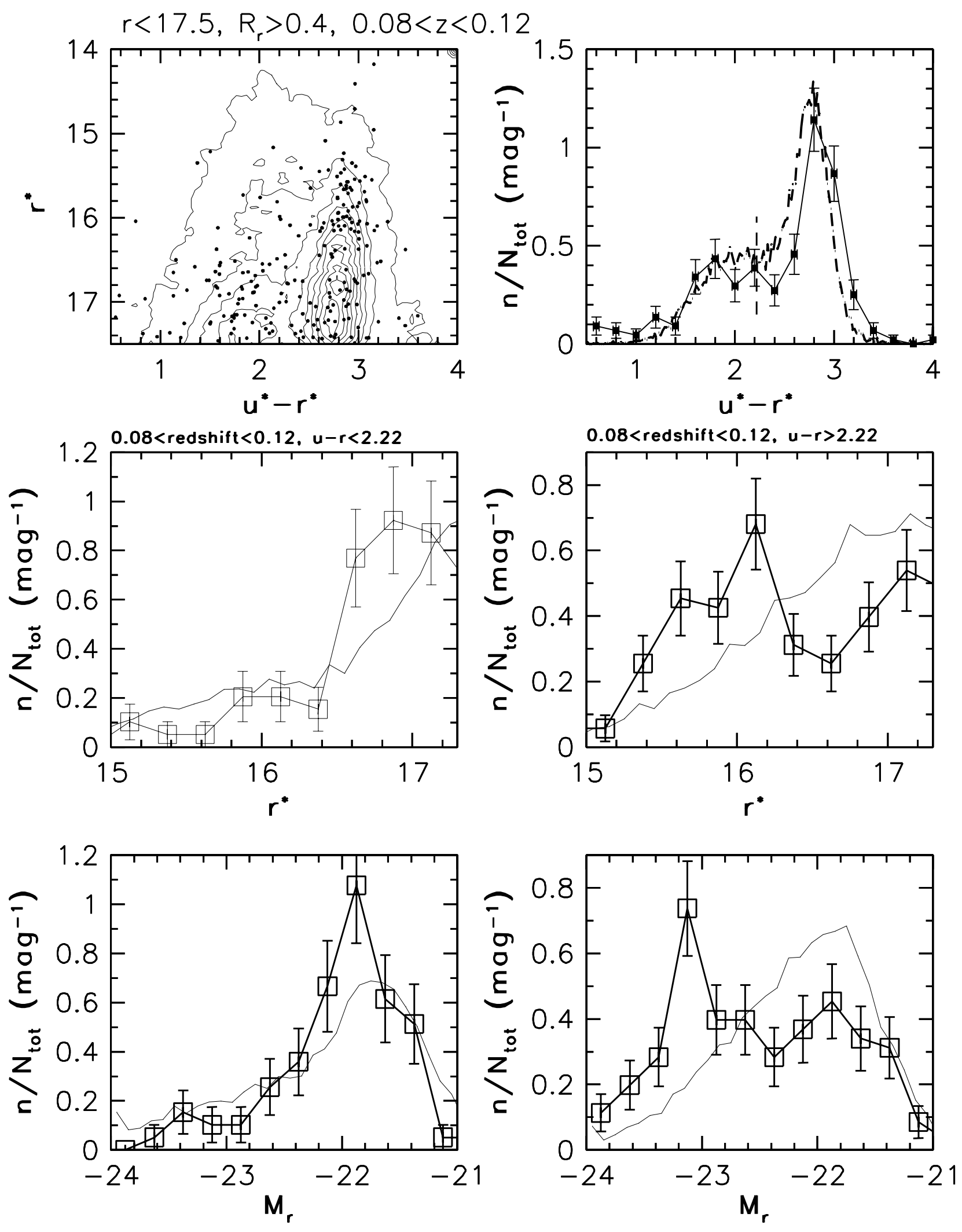

FIG. 27.-Top left: The $r^{*}$ vs. $u^{*}-r^{*}$ color-magnitude distribution for galaxies with $r^{*}<17.5$, redshift in the range $0.08-0.12$, and, for radio galaxies, $R_{r}>0.4 ; 219$ radio galaxies are shown by dots and all 31,226 galaxies by contours. Top right: Comparison of the $u^{*}-r^{*}$ histograms for radio (symbols) and all (dashed line) galaxies. Middle and bottom left: Comparison of the apparent and absolute magnitude distributions for blue $\left(u^{*}-r^{*}<2.22\right)$ galaxies and $(r i g h t)$ for red $\left(u^{*}-r^{*}>2.22\right)$ galaxies. All galaxies are marked by thin solid line, and radio galaxies by symbols. Radio galaxies have a different luminosity distribution from other galaxies. 
discussion of the $R_{r}$ distribution, see the next section). The top right panel compares the $u^{*}-r^{*}$ histograms. Despite the narrow redshift range the colors of red $\left(u^{*}-r^{*} \gtrsim 2.2\right)$ radio galaxies are $\sim 0.1 \mathrm{mag}$ redder than other galaxies from the same redshift range. This difference is larger than plausible $K$-correction variance due to the finite redshift bin size. As can be discerned from the top left panel, this effect is instead a consequence of a magnitude-color correlation: more luminous red galaxies are redder than less luminous red galaxies $^{47}$ (the slope is $\sim 0.1 \mathrm{mag} \mathrm{mag}^{-1}$ ). The small difference in color distributions between radio and other galaxies is due to their different magnitude distributions: radio galaxies tend to be brighter. This is better seen in the two middle panels, where we compare the apparent magnitude distributions for blue (left) and red (right) galaxies; all galaxies are shown by the thin solid line, and radio galaxies by symbols.

Both blue and red radio galaxies show a peak in their apparent magnitude distributions. The bottom two panels show the corresponding distributions of absolute magnitudes (we use the same cosmological parameters as in Appendix $\mathrm{C}$ and do not $K$-correct). As is evident, red radio galaxies show excess around $M_{r} \sim-23$, and blue radio galaxies around $M_{r} \sim-22$. We find that the radio fraction of red galaxies in the $-23.4<M_{r}<-22.8$ range $(1.2 \%)$ is about twice as high as the corresponding fraction for galaxies with $-22.4<M_{r}<-21.8(0.64 \%)$.

Interestingly, the luminosity difference between the two peaks ( $\sim 1 \mathrm{mag})$ is similar to the difference in characteristic luminosity between red and blue galaxies (Blanton et al. 2001). This suggests that the luminosity function of radio galaxies with a given color (or type) may be tied to the luminosity function of all galaxies with the same color.

\subsubsection{The Blue versus Red Galaxy Distribution in the Optical-Radio Flux Plane}

The distribution of radio galaxies in apparent magnitude versus color space is markedly different from the overall distribution of galaxies; in particular, the radio fraction of red galaxies is much higher than that of blue galaxies. This effect could be caused by intrinsic differences in radio emission properties between the two types of galaxies, or they could simply be subtle selection effects due to different luminosity distributions, flux limits, and $K$-corrections. Here we discuss these possibilities in more detail and provide qualitative arguments that explain the main trends in the data.

We demonstrated in preceding sections that the redshifts of radio galaxies tend to be larger than those of other galaxies in a flux-limited sample. This effect is probably due to a steeply rising radio luminosity function and the fact that the volume probed at higher redshift is larger, although we cannot exclude the possibility that this bias is partially caused by evolutionary effects. Similar effects can account, at least partially, for the different fractions of blue and red radio galaxies since they have different redshift distributions. Red galaxies are sampled at larger redshifts than blue galaxies (Fig. 26, bottom right) because the former tend to be more luminous than the latter. ${ }^{48}$ As a consequence of the

\footnotetext{
${ }^{47}$ This effect is not an artifact of SDSS data; for a similar result see for example Fig. 5 in Carlberg et al. (2001).

48 Blanton et al. (2001) find that galaxies with $M_{r} \lesssim-22$ are dominated by red galaxies (see also Madgwick et al. 2002 for consistent results based on the 2dF Galaxy Redshift Survey).
}

difference in redshifts the observed radio-to-optical flux ratio is greater for red galaxies, and thus they are preferentially detected by FIRST. We test this hypothesis by comparing the radio-to-optical flux ratio distributions for blue and red galaxies in narrow redshift bins. If the distributions are significantly different, then this hypothesis must be rejected.

Figure 28 shows the $t$ versus $r^{*}$ distributions for blue (top panels) and red (middle panels) galaxies brighter than $r^{*}=17.5$ and $t=16$, in two redshift ranges, 0.03-0.07 (left column), and 0.08-0.12 (right column). The three dashed lines in the top four panels show constant radio-to-optical flux ratios $R_{r}=0,1$, and 2, as marked. In each redshift range red galaxies are brighter in both the optical and the radio than blue galaxies; as a result their distributions of radio-to-optical flux ratio are very similar. This is better seen in the two bottom panels that show the $R_{r}$ distributions for objects from the strips defined by the two dot-dashed lines in each of the four top panels (note that, because of optical and radio flux limits, the $R_{r}$ distributions are unbiased only in the $0<R_{r} \lesssim 2$ range). This similarity indicates that the large differences in the observed radio fractions of blue and red galaxies may simply be due to different luminosity functions.

Windhorst et al. (1985 and references therein) show that radio sources with $t<13.9$ (flux density at $1.4 \mathrm{GHz}>10$ $\mathrm{mJy}$ ) consist predominantly of quasars and red giant elliptical galaxies, while only a few blue radio galaxies are seen. On the other hand, for $13.9<t<16.5$ blue radio galaxies become increasingly important. The data displayed in Figure 28 confirm these results. Furthermore, we show here that the distributions of radio-to-optical flux ratio for blue and red galaxies are very similar in the sampled range, $R_{r}>0$.

\subsubsection{The Luminous Red Galaxy Distribution in the Optical-Radio Flux Plane}

To further investigate the redshift dependence of the radio-to-optical flux ratio, we select additional subsamples from two larger redshift ranges: $0.28-0.32$ and $0.38-0.42$. Practically all the galaxies in these samples are luminous red galaxies; we caution that these samples are not flux limited but are limited by optical luminosity and color (E01). In this analysis we use $R_{z}$ instead of $R_{r}$ because of the large $K$-correction in the $r$ band. The median $i^{*}-z^{*}$ color of galaxies at these redshifts is only $\sim 0.1$ mag redder than the median $i^{*}-z^{*}$ color of nearby galaxies, indicating that the effects of optical $K$-correction are minimized in the $z$ band.

The distribution of galaxies from these two redshift ranges in the $t$ versus $z^{*}$ plane and their $R_{z}$ distributions are shown in Figure 29. The bottom panel demonstrates that $R_{z}$ is redshift dependent: the median $R_{z}$ is larger by $\sim 0.5$ for the larger redshift bin. Furthermore, both distributions show local maxima; i.e., we detect a radio dichotomy similar to that detected for quasars. The interpretation of this result requires detailed knowledge of radio spectral indices and thus will not be attempted here.

\subsection{The Spectral Properties of Radio Galaxies}

For practically all galaxies brighter than $r^{*} \sim 17.5$ (regions I in Fig. 22) the SDSS spectra are available (Strauss et al. 2002). In addition, many of the radio galaxies fainter than that limit also have spectra (region IId), because they are targeted for SDSS spectroscopy as the luminous red 

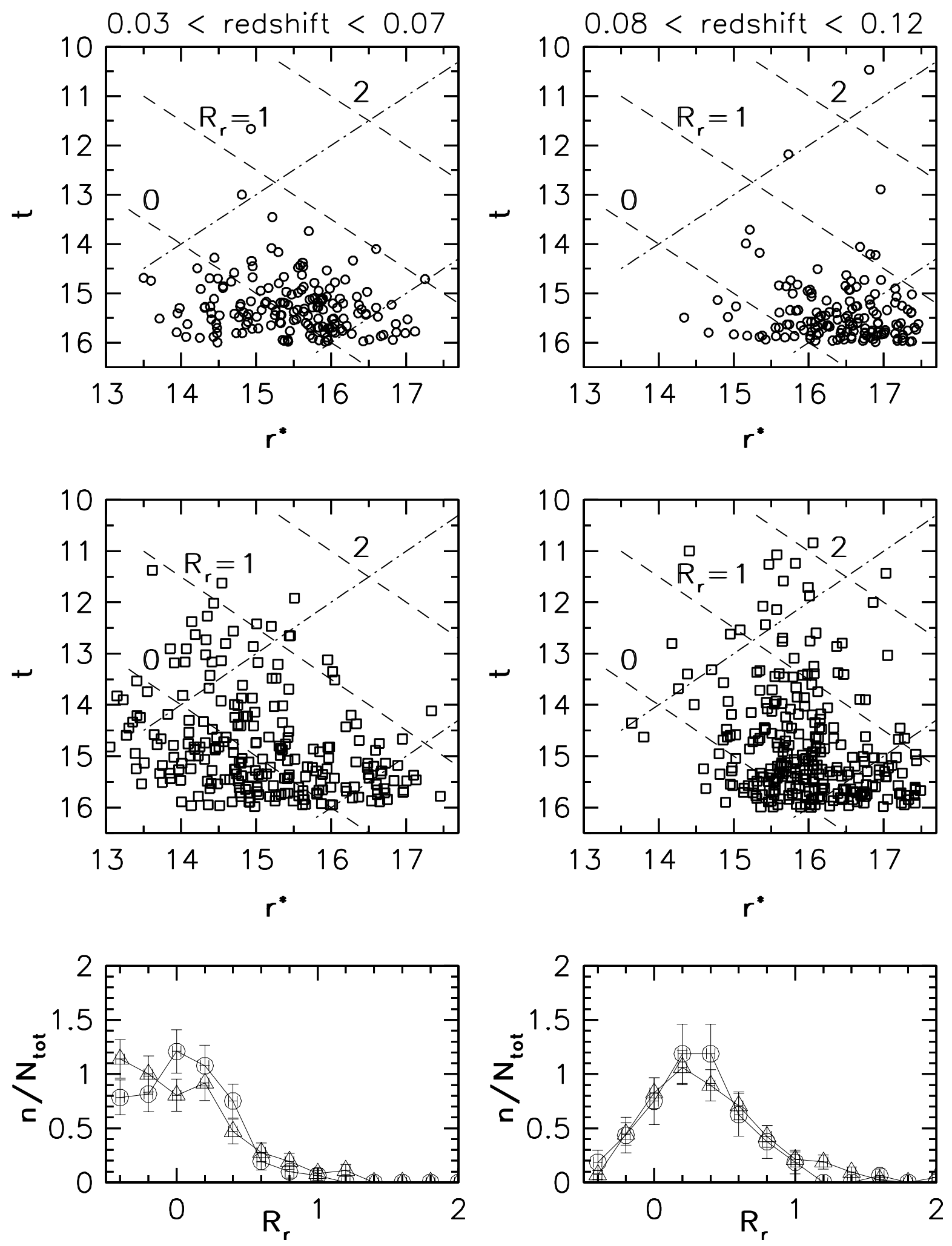

FIG. 28. - Distribution of SDSS-FIRST galaxies with $r^{*}<17.5$ and $t<16.0$, from two redshift slices $\left(0.03-0.07\right.$, left; and $0.08-0.12$, right) in the $t$ vs. $r^{*}$ diagrams. Top: Galaxies with $u^{*}-r^{*}<2.22$. Middle: Galaxies with $u^{*}-r^{*}>2.22$. The three dashed lines in the top four panels show constant radio-to-optical flux ratios of 0,1, and 2, as marked. Bottom: $R_{r}$ distributions for objects from the strip defined by the two dot-dashed lines in each panel; only $R_{r}>0$ points are complete. 

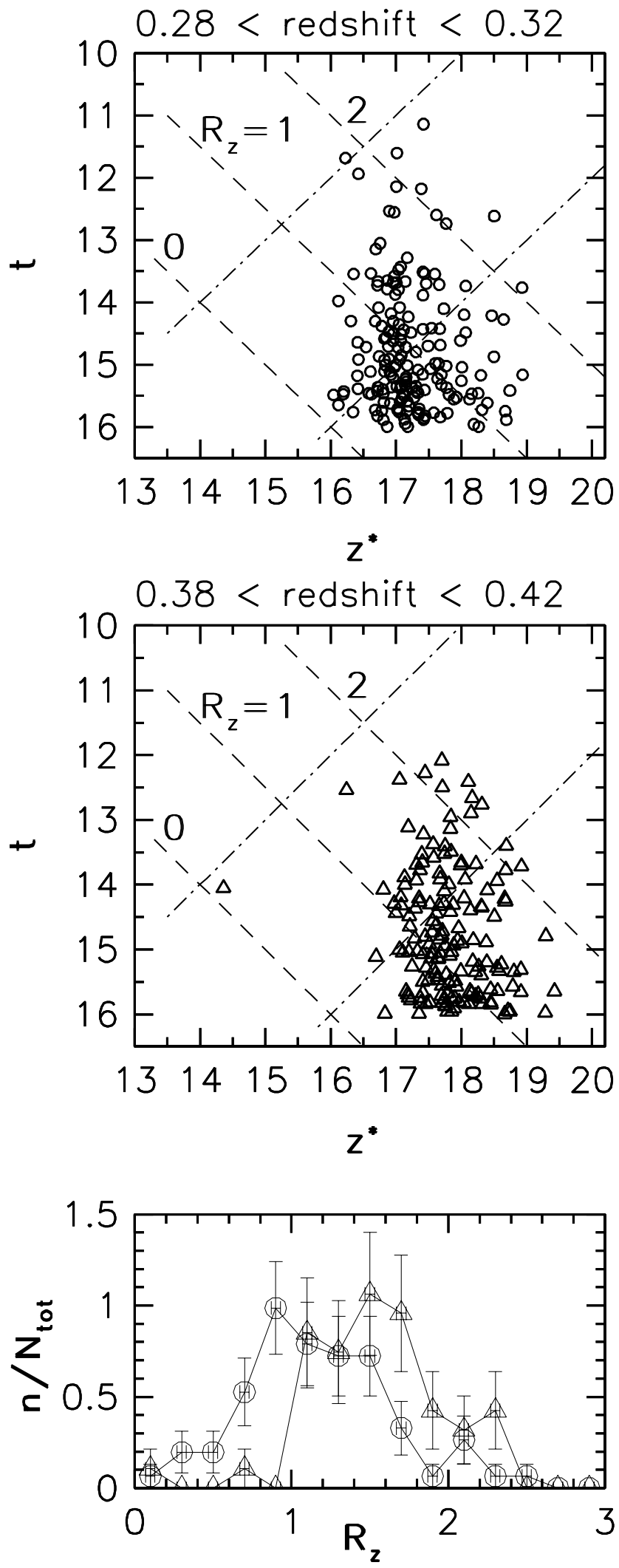

FIG. 29.-Distribution of SDSS-FIRST galaxies with $t<16.0$, from two redshift slices $(0.28-0.32$, top; and $0.38-0.42$, middle $)$ in the $t$ vs. $z^{*}$ diagrams. The three dashed lines in the top two panels show constant radio-to-optical flux ratios of 0,1 , and 2 , as marked. The bottom pane shows the $R_{z}$ distributions for objects from the strip defined by the two dot-dashed lines in the top panel (circles, lower redshift; triangles, larger redshift). galaxies (E01). At the time of this study spectra were available for 4300 SDSS-FIRST galaxies brighter than $r^{*}=21$, from $63 \%$ of the $1230 \mathrm{deg}^{2}$ area discussed here. The distribution of these galaxies in the $r^{*}$ versus $g^{*}-r^{*}$ color-magnitude diagram is shown in the bottom panel in Figure 22.

This is the largest homogeneous set of radio-galaxy spectra ever obtained. The largest previously available sample of 757 objects was produced by Sadler et al. (2002), who matched the $1.4 \mathrm{GHz}$ NRAO VLA Sky Survey (NVSS) and the 2dF Galaxy redshift Survey (Colless et al. 2001). A slightly smaller sample of 557 objects was obtained by Magliocchetti et al. (2002b), who matched the FIRST cata$\log$ with the 2dF Galaxy Redshift Survey. The SDSS spectra tend to have better quality than usual spectra acquired for redshift determination and hence can be used to measure the spectral properties of the galaxies.

\subsubsection{The Visual Inspection of Spectra}

Before we attempted the automated spectral classification described in the section, we visually inspected spectra for matched objects from the EDR sample, including 308 galaxies with $u^{*}-r^{*}<2.22$ and $r^{*}<17.5,112$ galaxies with $u^{*}-r^{*}>2.22$ and $r^{*}<17.5$, and 320 galaxies with $g^{*}-r^{*}>1.6$ and $r^{*}<19$. The last subsample was designed to include the luminous red galaxies with redshifts ranging from $\sim 0.35$ to $\sim 0.45$. Examples of spectra are shown in Figures 30 and 31 .

A substantial fraction ( $20 \%)$ of the 308 bright blue galaxies show spectra characteristic of starburst galaxies, and $\sim 10 \%$ show AGN-type spectra. The remaining blue galaxies show spectra typical for spiral galaxies (e.g., Kennicutt 1992). The majority of bright red galaxies show spectra characteristic of ordinary elliptical galaxies, with about onequarter classifiable as LINERs and a few percent indicating the presence of AGNs. The subsample of luminous red galaxies $^{49}$ with FIRST detections does not have significantly different spectra from the whole sample. There are clear cases of AGNs, though at a smaller rate than for the bright red galaxies. However, note that spectra from this subsample have lower signal-to-noise ratios due to a fainter magnitude limit, and thus AGNs may be more difficult to recognize. These results are in qualitative agreement with those of Sadler et al. (2002).

\subsubsection{The Fractions of AGNs and Starburst Galaxies}

A detailed quantitative study of spectral properties of the radio-galaxies from the SDSS-FIRST sample must be automated because of the large sample size. Here we present a preliminary determination of the fractions of AGNs and starburst galaxies, using diagnostic diagrams based on the strengths of several emission lines. Details of the line strength determination will be presented elsewhere (Tremonti et al. 2002).

Galaxies with emission lines due to strong star formation can be separated from galaxies whose emission lines originate from an AGN by using optical line diagnostic diagrams. Such a separation is possible because AGNs have a much harder ionizing spectrum than stars. This method was first proposed by Baldwin, Phillips, \& Terlevich (1981) and

\footnotetext{
${ }^{49}$ The luminous red galaxies are those that have large bright absolute magnitude, as opposed to the bright red galaxies that have bright apparent magnitude.
} 


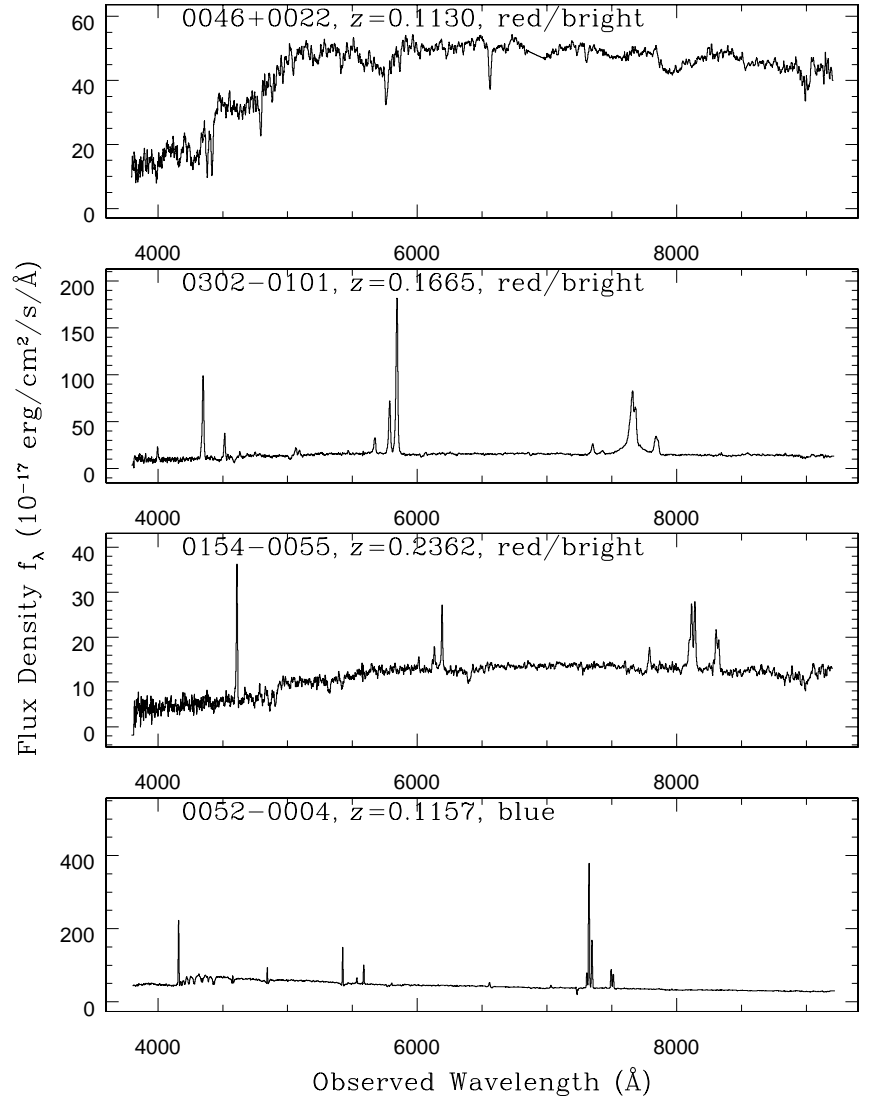

FIG. 30.-Examples of SDSS spectra for radio galaxies discussed here. The galaxy name, redshift, color and brightness is marked in each panel.

was further developed semiempirically by Osterbrock \& de Robertis (1985) and Veilleux \& Osterbrock (1987), and theoretically by Kewley et al. (2001). The line diagnostic diagrams are constructed with the line strength ratios $[\mathrm{O} \mathrm{III}] / \mathrm{H} \beta,[\mathrm{N}$ II] $/ \mathrm{H} \alpha,[\mathrm{S}$ II] $/ \mathrm{H} \alpha$, and $[\mathrm{O} \mathrm{I}] / \mathrm{H} \alpha$. We computed these ratios for galaxies with $r^{*}<17.5$; the samples with unsaturated and better than $3 \sigma$ detections for $\mathrm{H} \alpha, \mathrm{H} \beta$, [O III(5007)], [N II(6584)], and [S II(6717 + 6731)] include 650 SDSS-FIRST galaxies and 16,325 SDSS galaxies (we do not use the [O I] line because its $3 \sigma$ requirement would significantly reduce the sample size). These subsamples represent $26 \%$ and $18 \%$, respectively, of all galaxies for which the line strength measurement was attempted.

The distribution of the 16,325 SDSS galaxies in the [N II]/ $\mathrm{H} \alpha$ versus $\left[\mathrm{O}{ }_{\mathrm{III}}\right] / \mathrm{H} \beta$ and $[\mathrm{S} \mathrm{II}] / \mathrm{H} \alpha$ versus $[\mathrm{O} \mathrm{III}] / \mathrm{H} \beta$ diagrams is shown in Figure 32. The distribution of galaxies shows a remarkable structure in these diagrams, rather than a random scatter ${ }^{50}$ the overall distribution is in agreement with previous work that was based on much smaller samples (e.g., Veilleux \& Osterbrock 1987 and references therein). The dashed lines, obtained theoretically by Kewley et al. (2001), separate AGNs from starburst galaxies. Motivated by the distribution of galaxies in the $[\mathrm{N} \mathrm{II}] / \mathrm{H} \alpha$ versus $[\mathrm{O} \mathrm{III}] / \mathrm{H} \beta$ diagram, we place the additional constraint that $[\mathrm{N} \mathrm{II}] / \mathrm{H} \alpha>-0.5$ for a source to be classified as an AGN,

\footnotetext{
${ }^{50}$ We do not find significant changes in the morphology of data distribution in these diagrams for various subsamples selected from narrow redshift and apparent magnitude bins.
}

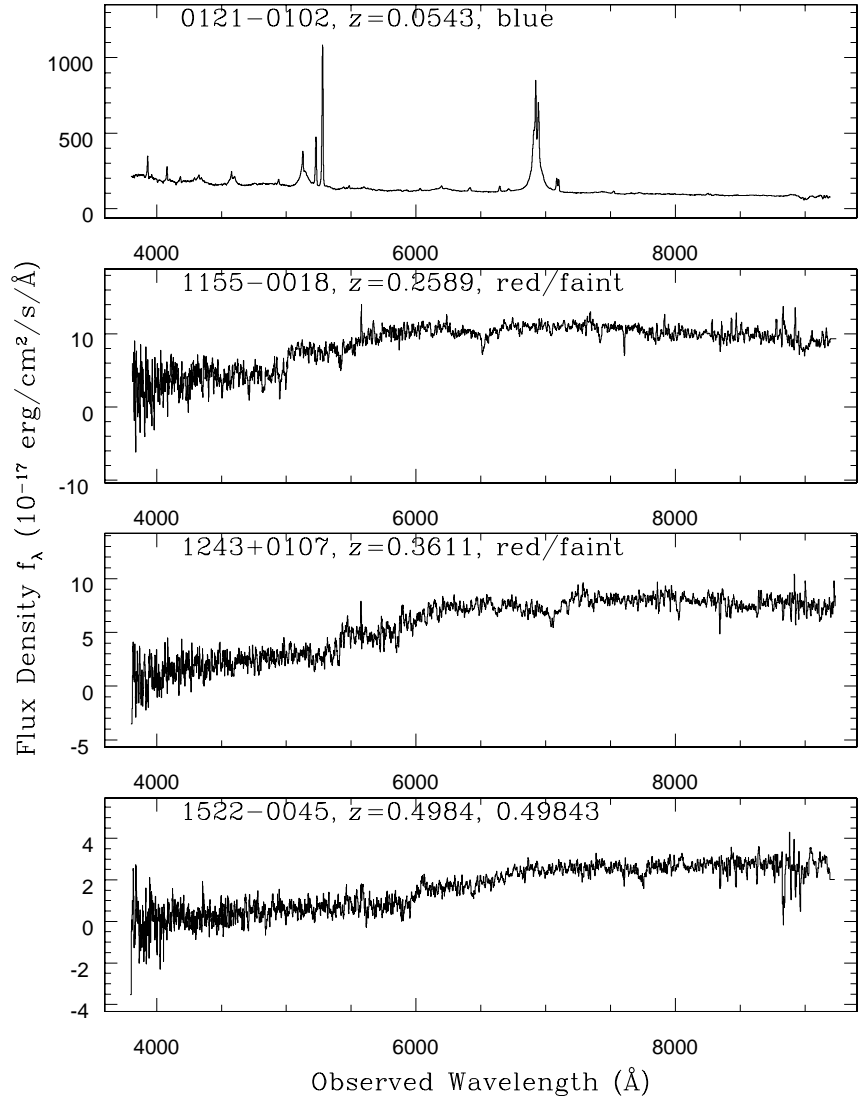

FIG. 31.-Additional examples of SDSS spectra for radio galaxies discussed here. The galaxy name, redshift, color, and brightness is marked in each panel.

to exclude a small number of low-metallicity starbursts. Requiring the same classification in both diagrams removes only $\sim 7 \%$ of galaxies. We find that $5 \%$ of all galaxies are classified as AGNs and $88 \%$ as starbursts, implying a starburst-to-AGB ratio of $\sim 18$ in the full sample. The data for the 650 radio-galaxies are shown in Figure 32 as circles; the corresponding starburst-to-AGN number ratio for radiogalaxies is 2.4 , significantly smaller than for the full sample. That is, the fraction of radio galaxies whose emission-line ratios indicate an AGN rather than a starburst origin is $\sim 6$ times larger than the corresponding fraction for all SDSS galaxies.

\subsubsection{The Optical Colors of Galaxies with Strong Emission Lines}

The galaxies with strong emission lines that are analyzed here are predominantly blue. The top panel in Figure 33 shows the $r^{*}$ versus $u^{*}-r^{*}$ distribution of the 16,325 SDSS galaxies as contours (compare with the top left panel in Fig. 26). Their $u^{*}-r^{*}$ color distribution is shown separately in the middle panel, for AGNs (long-dashed line) and starburst galaxies (short-dashed line). Note that each curve is separately normalized. AGNs have redder $u^{*}-r^{*}$ colors by $\sim 0.6$ mag than do starburst galaxies. The bottom panel compares their redshift distributions using analogous notation. The $u^{*}-r^{*}$ color and redshift distributions for radio-galaxies are shown by dots for starburst galaxies and by triangles for AGNs. There is no significant difference in color and redshift distributions between radio galaxies and the full 

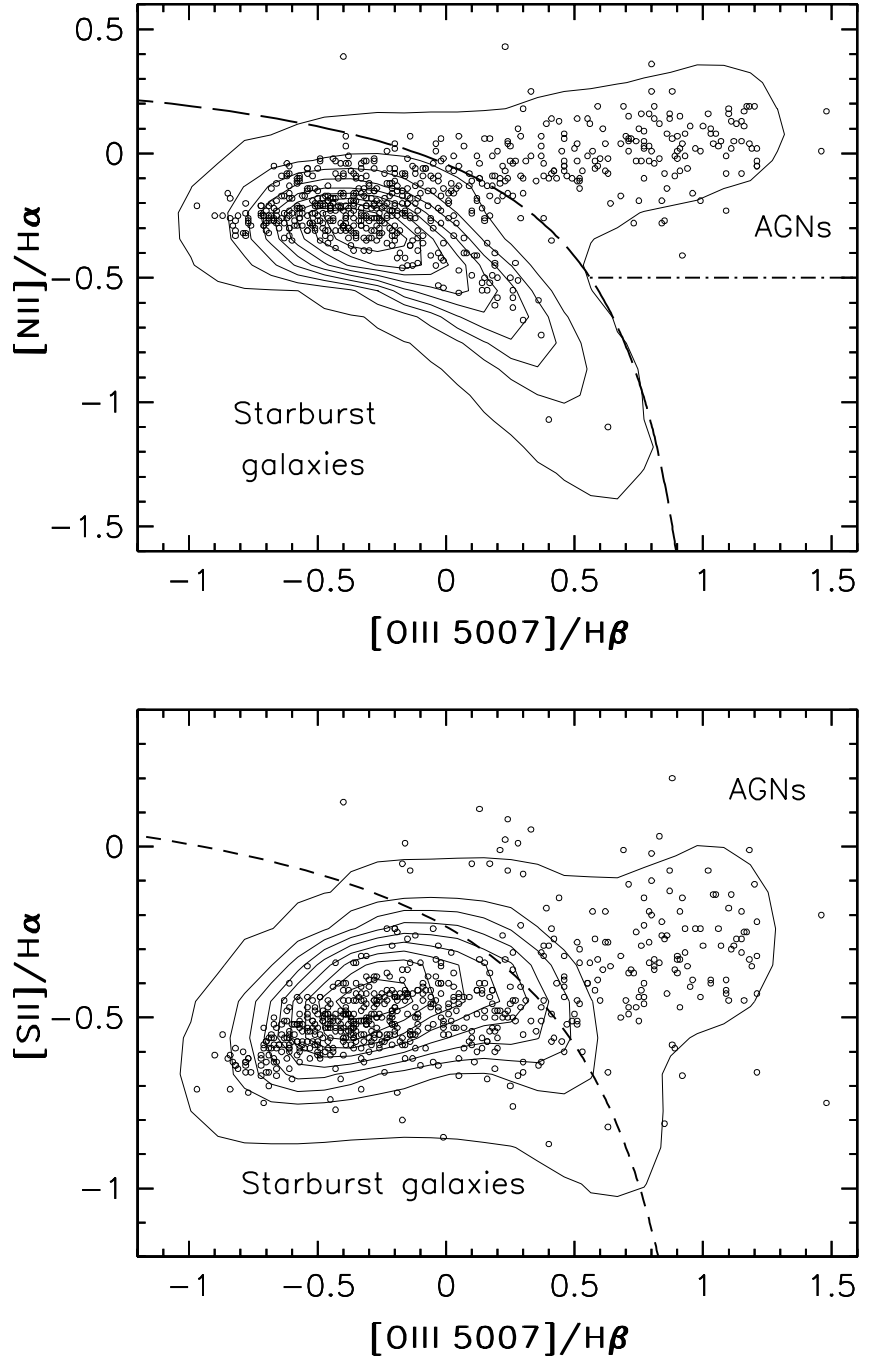

FIG. 32.-Line diagnostic diagrams separating AGNs and starburst galaxies. The distributions of 16,325 SDSS galaxies with $r^{*}<17.5$ are shown by linearly spaced contours. The 650 SDSS-FIRST galaxies selected by the same criteria are shown as circles. The dashed lines, obtained theoretically by Kewley et al. (2001), and the dot-dashed line in the top panel separate AGNs from starburst galaxies. There are $~ 18$ starburst galaxies for each AGN in the entire SDSS sample; for the SDSS-FIRST sample this ratio is $\sim 2.4$.

sample. In particular, radio galaxies show similar separation of $u^{*}-r^{*}$ color between starburst and AGN types.

\subsubsection{The Radio Properties of Galaxies with Strong Emission Lines}

We examine next the radio properties of the two classes of radio galaxies. The top panel in Figure 34 compares the differential counts as a function of the radio magnitude, and the bottom panel displays the distributions of the radio concentration parameter $\theta$ for sources with $t<15$. Radio galaxies classified as starbursts are marked by circles, and AGNs by triangles. Their cumulative counts for $t<15$ are similar, while there are 3 times more starburst galaxies than AGNs in the $15<t<16$ range. This increase of the starburst galaxy-to-AGN number ratio as the radio flux decreases is consistent with the known differences in their radio luminosity functions (Machalski \& Godlowski 2000; Sadler et al. 2002). Machalski \& Godlowski detected a turnover in AGN
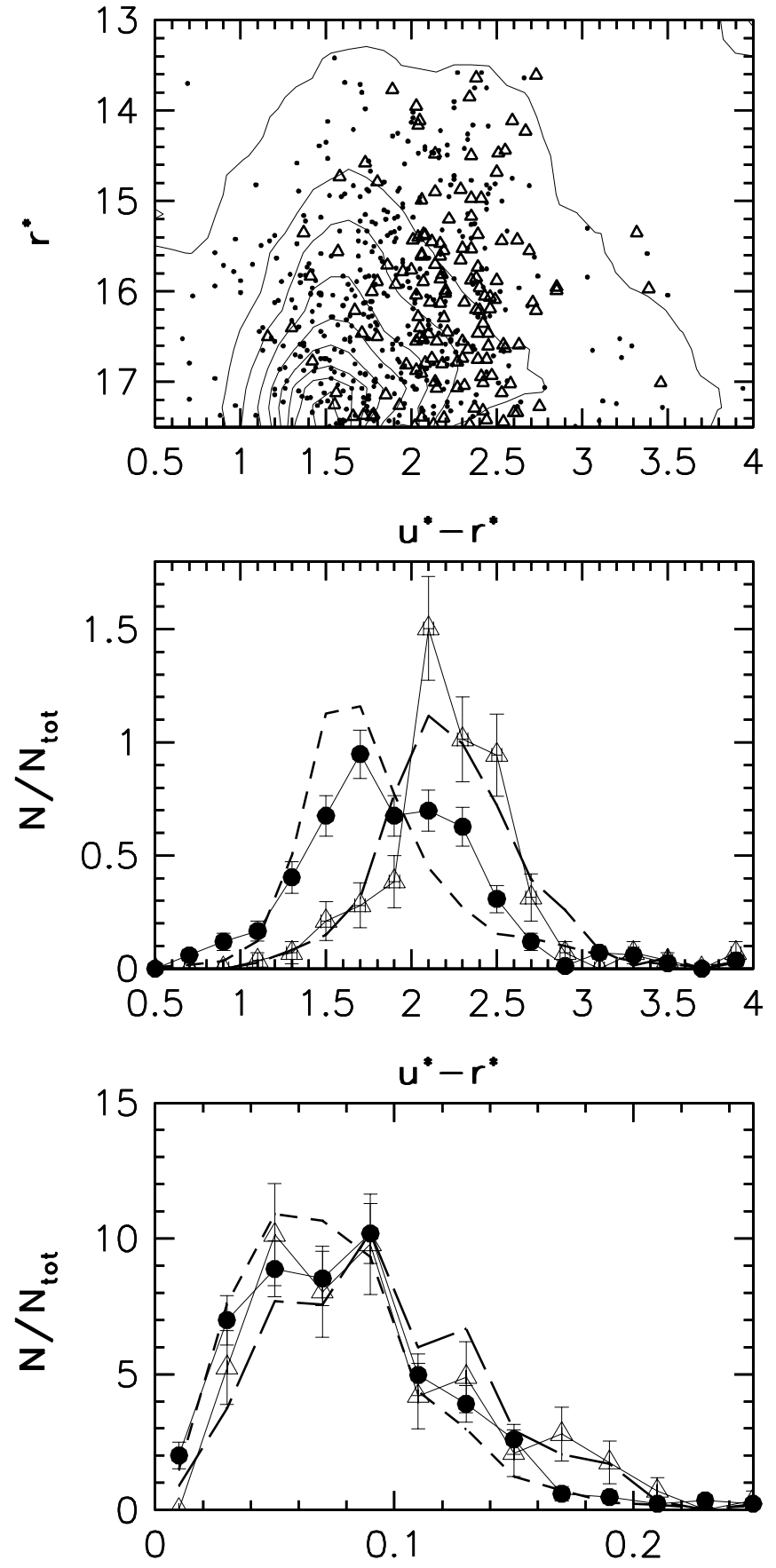

redshift

FIG. 33.-Comparison of the magnitude, color, and redshift distributions for SDSS-FIRST galaxies classified as AGN (143, triangles) and starburst galaxies $(422$, dots) using the line diagnostic diagrams. The distribution of the control sample of SDSS galaxies is shown by contours in the top panel and by the short-dashed (starburst galaxies) and long-dashed (AGNs) lines in the other two panels. AGNs are redder than starbursts by $\sim 0.6$ mag for both radio sample and the control sample.

counts at the faint radio end, an effect not seen by Sadler et al. The data discussed here seem to support the claim by Machalski \& Godlowski. Furthermore, recent deep radio imaging by Fomalont et al. (2002) also suggests that there is an increasing contribution from starburst galaxies compared with AGNs at faint radio levels. 

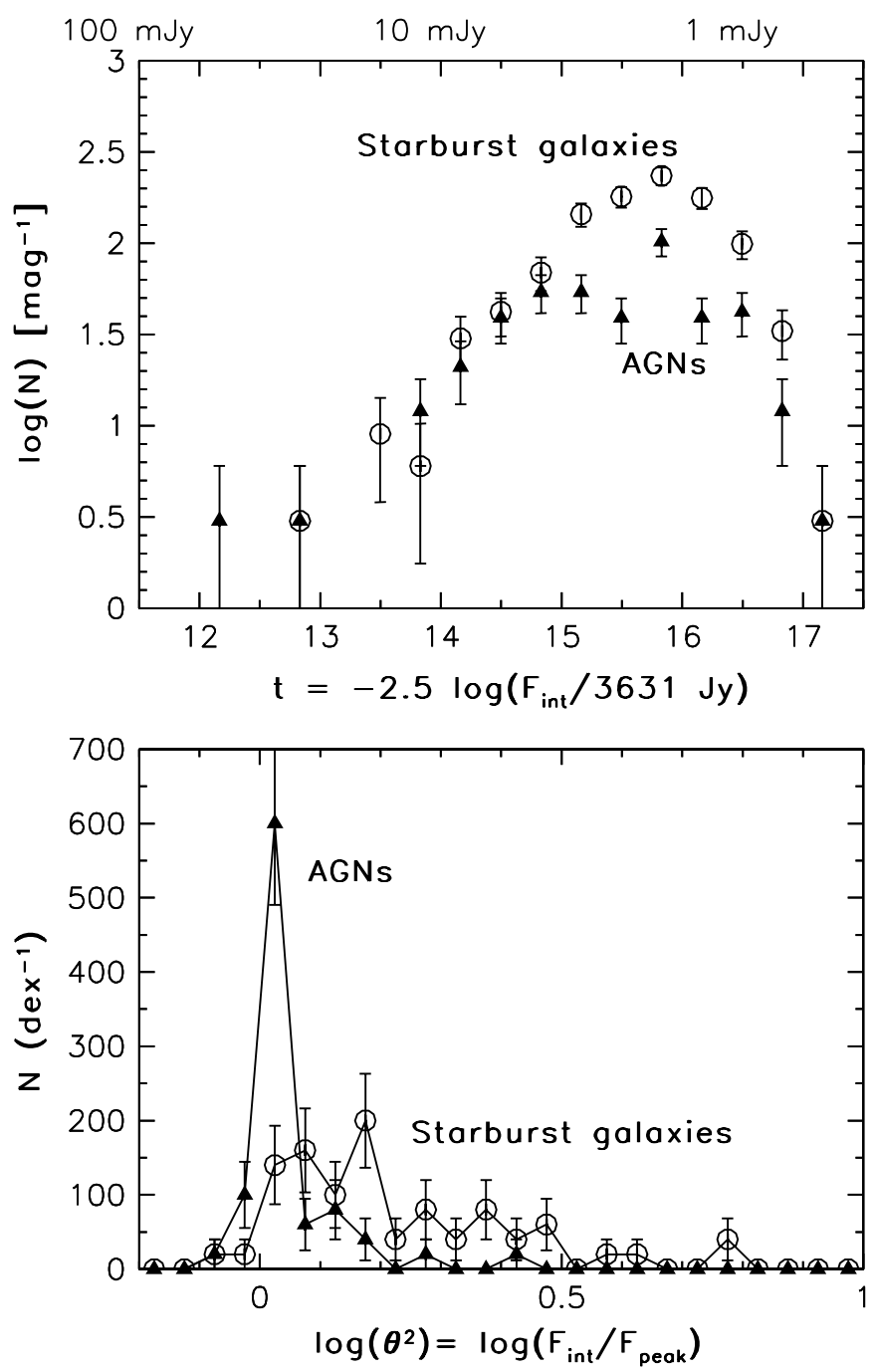

FIG. 34.-Comparison of the radio properties for SDSS-FIRST galaxies classified as AGNs (triangles) and starburst galaxies (circles) using emission-line strengths. Top: Comparison of the differential counts as a function of radio magnitude. Bottom: Distributions of the radio concenBtration parameter $\theta$ for sources with $t<15$. The cumulative counts for $t<15$ are similar, while there are 3 times more starburst galaxies than AGNs in the $15<t<16$ range. Starburst galaxies tend to have larger radio concentration parameters than do AGNs.

The bottom panel in Figure 34 compares the distributions of the radio concentration parameter. As is evident, starburst galaxies tend to have a larger radio concentration parameter than AGNs, in agreement with the expected nuclear origin of AGN emission. This difference strongly supports the robustness of the AGN-starburst galaxy separation, which is fully based on optical spectral properties; its detection is possible due to our large spectroscopic sample and the good spatial resolution of the FIRST survey.

It is possible that the high spatial resolution achieved by FIRST affects the radio counts ${ }^{51}$ plotted in Figure 34. We reproduce these counts using NVSS instead of FIRST in Figure 35. Since the NVSS faint cutoff is brighter than the FIRST cutoff, the magnitude distribution does not extend as deep as in Figure 34. Nevertheless, the plotted histograms

${ }^{51}$ We thank E. Sadler for pointing out this concern to us.
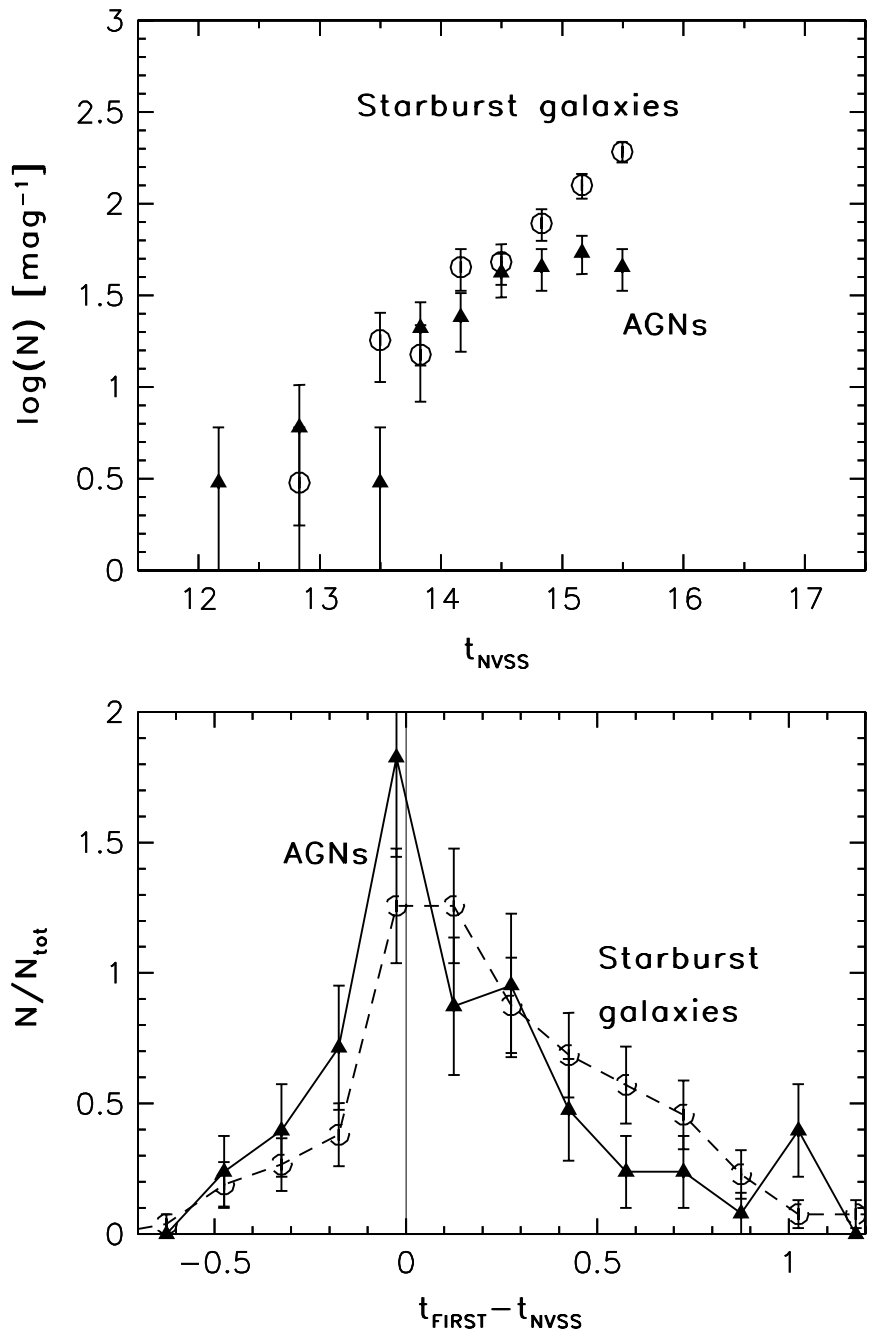

FIG. 35.-Top: Same as in Fig. 34, except that NVSS is used instead of FIRST. Note that the AGN counts appear to turn over around $t \sim 15$, while the counts of starburst galaxies continue to steadily rise. Bottom: Comparison of the distribution of differences between FIRST and NVSS measurements for AGNs and starburst galaxies.

support the conclusion that the AGN counts turn over around $t \sim 15$, while the counts of starburst galaxies continue to steadily rise. The bottom panel compares the distribution of $t_{\mathrm{FIRST}}-t_{\mathrm{NVSS}}$ difference for AGN and starburst galaxies. There is marginal evidence that this difference is slightly larger for the latter.

\subsubsection{A Strategy for Selecting $z>0.5$ Galaxy Candidates Using SDSS and FIRST}

The farthest galaxies targeted for SDSS spectroscopy are the luminous red galaxies (E01). The targeting strategy is based on $g^{*}-r^{*}$ and $r^{*}-i^{*}$ colors as a function of magnitude and extends down to $r^{*} \sim 19.5$, yielding about 12 galaxies $\mathrm{deg}^{-2}$ at redshifts up to $\sim 0.55$. Galaxies at such large redshifts are important for studies of large-scale structure and galaxy evolution, and their usefulness increases with redshift. The selection of galaxies at even larger redshifts can rely only on $r^{*}-i^{*}$ and $i^{*}-z^{*}$ colors because their $g$-band flux is too small to be well-measured. However, the efficiency of such color selection is low because of large photometric errors and errors in star-galaxy separation, 

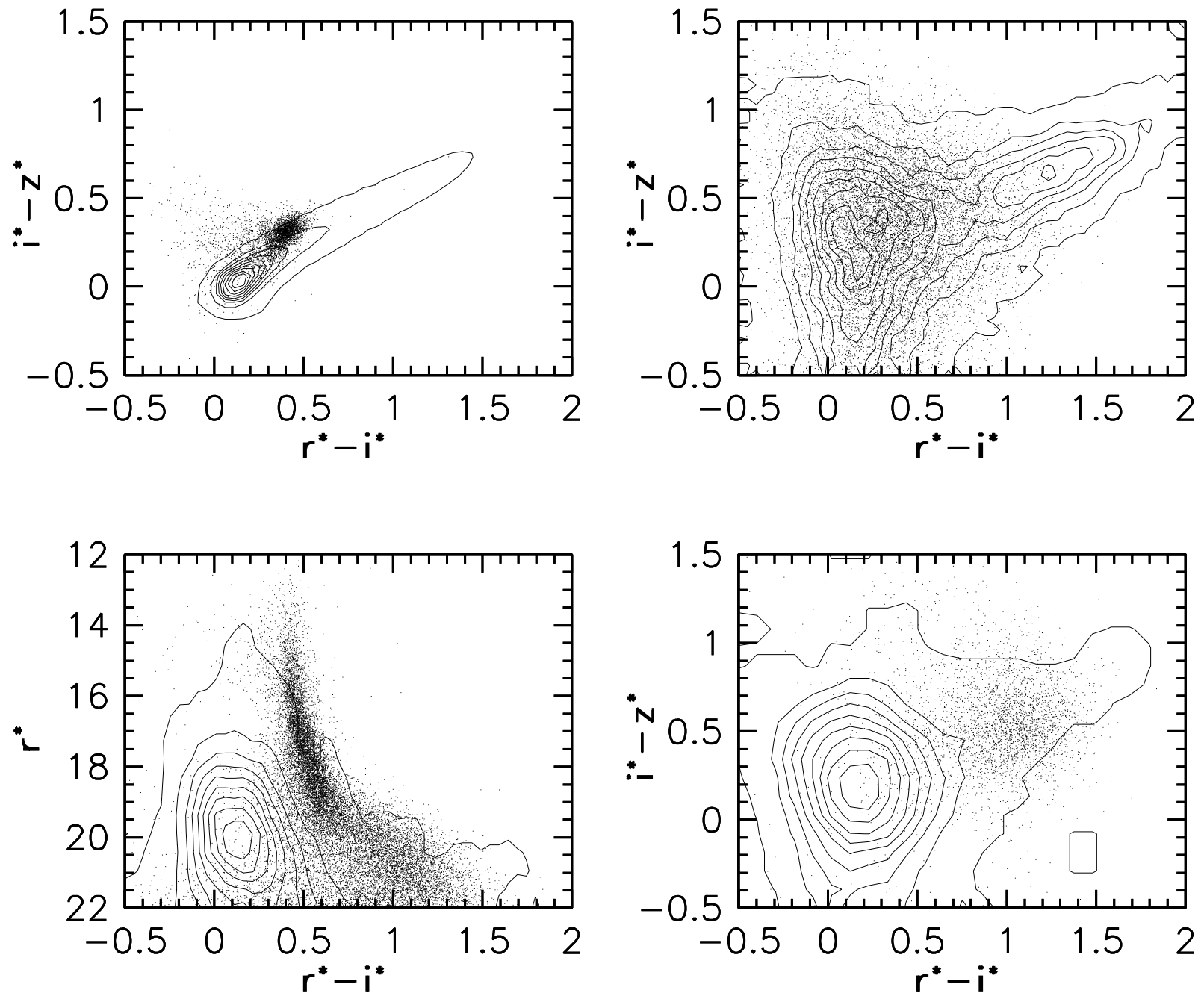

FIG. 36. - Top: The $i^{*}-z^{*}$ vs. $r^{*}-i^{*}$ color-color diagrams for SDSS sources from $\sim 100 \mathrm{deg}^{2}$ of sky and selected from two magnitude bins: $r^{*}<17.5$ (left, $\sim 20,000$ sources) and $21.4<r^{*}<21.5$ (right, $\sim 20,000$ sources). The distribution of unresolved sources is shown as linearly spaced contours, and resolved sources (dots). Bottom: Distribution of $\sim 22,000$ SDSS-FIRST sources from $1230 \mathrm{deg}^{2}$ of sky in the $r^{*}$ vs. $r^{*}-i^{*}$ and $i^{*}-z^{*}$ vs. $r^{*}-i^{*}$ diagrams (same notation).

since most of high-redshift galaxy candidates are faint $\left(r^{*} \lesssim 21.5\right)$. We show here that the selection efficiency can be increased by combining SDSS and FIRST data. Since the number of radio stars is insignificant, they are not a serious source of contamination.

The $r^{*}-i^{*}$ colors of SDSS galaxies at redshifts above $\sim 0.4$ correlate well with their redshifts. By fitting the $r^{*}-i^{*}$ color-redshift distribution shown in the bottom right panel ${ }^{52}$ in Figure 25, we find that a best-fit relation

$$
\text { redshift }=0.54\left(r^{*}-i^{*}\right)+0.02
$$

produces redshifts within 0.05 (rms scatter) from the spectroscopically measured values for $0.7<r^{*}-i^{*}<1.5$ $(0.4<$ redshift $<0.8)$. Thus galaxies with redshifts in the range $0.5-0.8$ can be selected by requiring $0.9<r^{*}-i^{*}<1.5$. The median $i^{*}-z^{*}$ color of both spectroscopically confirmed galaxies and of SDSS-FIRST galaxies with such $r^{*}-i^{*}$ colors is $\sim 0.5-0.6$. This region overlaps

\footnotetext{
52 Most of the galaxies at redshifts larger than $\sim 0.55$ with spectra were selected as high-redshift quasar candidates and FIRST quasar candidates (for details see Richards et al. 2002).
}

with the stellar locus in the $i^{*}-z^{*}$ versus $r^{*}-i^{*}$ color-color diagram.

To illustrate the effect of increased photometric errors close to the faint end, we show in the top two panels in Figure 36 the $i^{*}-z^{*}$ versus $r^{*}-i^{*}$ color-color diagrams for sources selected in two magnitude bins. The top left panel shows 20,000 sources with $r^{*}<17.5$ from $\sim 100 \mathrm{deg}^{-2}$ of sky, and the top right panel shows $\sim 20,000$ sources with $21.4<r^{*}<21.5$ from the same region. The faint unresolved and resolved sources fully overlap in the $i^{*}-z^{*}$ versus $r^{*}-i^{*}$ color-color diagram.

The surface density of SDSS sources with $0.9<r^{*}-i^{*}<1.5$ and $19<r^{*}<21.5$ is $\sim 334 \mathrm{deg}^{-2}$ for unresolved sources and $\sim 65 \mathrm{deg}^{-2}$ for resolved sources. ${ }^{53}$ The repeatability of the star-galaxy separation is at the level of $90 \%$ for $r^{*} \sim 21$ and further deteriorates at the fainter levels. If all sources with colors appropriate for galaxies at $z \gtrsim 0.5$ were targeted, the expected efficiency would be well below $50 \%$.

\footnotetext{
${ }^{53}$ Galaxies outnumber stars in SDSS data at $r^{*} \sim 21$. However, the opposite is true in the relevant narrow range of $r^{*}-i^{*}$ color.
} 
A higher targeting efficiency can be achieved for radio galaxies. As shown by K02, the fraction of red stars detected both by SDSS and FIRST is very small, and the condition that a candidate is detected by FIRST effectively rejects all the stellar contaminants. The distribution of SDSS-FIRST sources from $1230 \mathrm{deg}^{2}$ of sky in the $r^{*}$ versus $r^{*}-i^{*}$ and $i^{*}-z^{*}$ versus $r^{*}-i^{*}$ diagrams is shown in the bottom two panels in Figure 36. The selection condition $0.9<r^{*}-i^{*}<1.5$ and $19<r^{*}<21.5$ yields 3.2 sources $\operatorname{deg}^{-2}$. Thus matched SDSS and FIRST catalogs could be used to select a well-defined sample of 32,000 radio galaxies at redshifts in the range $0.5-0.8$ (over the eventual survey area of 10,000 $\mathrm{deg}^{2}$ ).

Such a sample would be of great importance for studies of galaxy evolution and for detecting clusters of galaxies. Clusters discovered at such large redshifts provide strong upper limits on the mass density parameter of the universe, $\Omega_{M}$, and on the amplitude of mass fluctuations, $\sigma_{8}$ (Bahcall \& Fan 1998 and references therein). Such a sample could be cross-correlated with distant cluster candidates selected by other methods, e.g., by the matched filter method (Kim et al. 2002), or surface brightness fluctuations method (Dalcanton 1996; Bartelmann \& White 2002), thus increasing the reliability of the matched candidates.

\section{DISCUSSION}

This preliminary analysis of the objects detected by both SDSS and FIRST indicates the enormous potential of combining large-scale surveys at different wavelengths. The final photometric SDSS-FIRST catalog, including five-color accurate optical photometry and morphological information and radio data complete to $1 \mathrm{mJy}$ level, will be available for $\sim 200,000$ radio galaxies and $\sim 40,000$ radio quasars. SDSS spectra will be available for about 50,000 radio galaxies and 15,000 radio quasars; both surveys will provide outstanding astrometry ( $\sim$ ". 1 for SDSS and $\sim 0$ ".4 for FIRST) for an unprecedented number of objects. Such a large, detailed, and accurate data set will certainly place studies of the properties of extragalactic radio sources on a new level.

The main results presented here are as follows:

1. We discuss optical and radio properties of $\sim 30,000$ FIRST sources positionally matched within 1 ". 5 to an SDSS source in $1230 \mathrm{deg}^{2}$ of sky. The matched sample represents $\sim 30 \%$ of the $\sim 108,000$ FIRST sources and $0.1 \%$ of the $2.5 \times 10^{7}$ SDSS sources in the studied region. SDSS spectra are available for 4300 galaxies and 1154 quasars from the matched sample.

2. Differential radio counts of FIRST sources with and without SDSS counterparts have indistinguishable slopes; about $25 \%$ of FIRST sources are associated with an SDSS source brighter than $r^{*}=21$ ( $\$ 3.5$ and Fig. 6). This similarity, given the different number count slopes of galaxies and quasars, suggests that the quasar-to-galaxy number ratio $(\sim 1: 5)$ may be comparable for SDSS-FIRST and FIRSTonly radio sources.

3. The fraction of SDSS-FIRST sources with complex radio morphology is less than $10 \%$. For optically resolved sources double-lobe morphology is the dominant type, while for unresolved sources the fractions of core-lobe and double-lobe morphological types appear comparable.
4. The majority of SDSS-FIRST sources brighter than $r^{*}=21$ are optically resolved. The fraction of resolved objects among the matched sources is a function of the radio flux, increasing from $\sim 50 \%$ at the bright end to $\sim 90 \%$ at the FIRST faint limit ( 33.7 and Fig. 8).

5. Most optically unresolved radio sources have nonstellar colors indicative of quasars. We estimate an upper limit of $\sim 5 \%$ for the fraction of quasars with colors indistinguishable from those of stars and thus missed by SDSS spectroscopic quasar survey (§ 4.1.1 and Fig. 14). However, a subset of those detected by FIRST are targeted for SDSS spectroscopic observations and will yield a large number ( $\gtrsim 1000$ ) of quasars with unusual spectra (Fig. 15).

6. We find statistically significant differences in the optical color distribution between radio-loud and radio-quiet quasars selected from the same redshift range: the radioloud quasars have a redder median color by $\sim 0.1$ mag and show a 3 times larger fraction of objects with extremely red colors ( $\S 4.1 .2$ and Fig. 16). The distributions of optical spectral indices also indicate that spectra of radio-loud quasars tend to be redder than spectra of radio-quiet quasars (Fig. 17).

7. The fraction of optically identified quasars that is detected by FIRST decreases with optical brightness from $\sim 50 \%$ for $r^{*} \sim 17$ to $\sim 10 \%$ for $r^{*} \sim 20$; this decrease is a selection effect caused by the radio sensitivity limit $(\S 4.1 .3$ and Fig. 18).

8. The distribution of quasars in the radio flux-optical flux plane suggests the reality of the "quasar radio dichotomy "; $8 \% \pm 1 \%$ of all quasars with $i^{*}<18.5$ are radio-loud $\left(R_{i}>1\right)$, and this fraction seems independent of redshift and optical luminosity ( $\$ 4.2$ and Figs. 19 and 21).

9. FIRST galaxies represent $5 \%$ of all SDSS galaxies with $r^{*}<17.5$ and $1 \%$ for $r^{*}<20$ and are dominated by red galaxies, especially those with $r^{*}>17.5$ (§ 5.1, Table 1 , and Fig. 22). This difference between blue and red galaxies appears to be a selection effect due to their different luminosity functions. In particular, the distribution of the radio-tooptical flux ratio for galaxies selected from narrow redshift bins is indistinguishable for blue and red galaxies (Fig. 28).

10. Radio galaxies have a different optical luminosity distribution than other galaxies selected by the same redshift and optical brightness criteria; when galaxies are further separated by their colors, this result remains valid for each color type.

11. Radio-galaxies in luminosity and redshift-limited samples have indistinguishable colors from other galaxies selected by identical criteria. In optical and radio fluxlimited samples radio-galaxies are biased toward larger redshifts and thus have redder observed colors due to optical $K$ corrections ( $\$ 5.1$ and Figs. 25 and 26).

12. The fraction of radio galaxies whose emission-line ratios indicate an AGN rather than a starburst origin $(30 \%)$ is 6 times larger than the corresponding fraction for all SDSS galaxies ( $\$ 5.2$ and Figs. 32 and 33). The AGN and starburst galaxies classified using optical spectra have distinct radio properties. The AGN-to-starburst count ratio increases with radio flux, and AGNs tend to have more concentrated radio emission than starburst galaxies (Fig. 34).

13. FIRST and SDSS data can be used to efficiently select galaxies at redshifts between 0.5 and 0.8 with a surface density of $\sim 3 \mathrm{deg}^{-2}$ for candidates with $r^{*}<21.5$ (§ 5.2.5 and Fig. 36). Such a sample would be of great importance for 
studies of galaxy evolution and for detecting clusters of galaxies.

\subsection{Future Work}

\subsubsection{FIRST-only and SDSS-only Sources}

The sample discussed here provides a good measure of the $R_{i}$ distribution for radio-loud quasars. To obtain a commensurate description of the $R_{i}$ distribution for radio-quiet quasars, radio observations significantly more sensitive than the FIRST survey are needed. Based on a sample of $\sim 100$ sources observed by Kellermann et al. (1989), the required sensitivity gain is about a factor of $10-100$. Of course, obtaining data in more than one band would be extremely valuable (see Appendix D).

About two-thirds of the FIRST sources are not detected by SDSS. The counts of quasars in the FIRST-only sample can be compared with deep optical counts of quasars $\left(i^{*} \lesssim 25\right)$ to constrain the number of anomalously optically faint objects missed in optical surveys (see Appendix B). Thus deep optical observations of regions containing FIRST sources without SDSS counterparts, preferably in two or more bands (§3.6.2), would be very valuable.

\subsubsection{Multiwavelength Observations of SDSS-FIRST Sources}

SDSS and FIRST data span a wide wavelength range, but significant parts of the spectrum remain unexplored. The available multiwavelength catalogs of extragalactic sources that include X-ray, IR, and submillimeter data are much smaller than the sample discussed here. The matching of SDSS-FIRST sources to sensitive large-scale sky surveys at other wavelengths is thus of obvious importance. As an example, we consider the upcoming SIRTF observations. The SIRTF First Look Survey will observe a $\sim 4 \mathrm{deg}^{2}$ large region $^{54}$ that overlaps with the SDSS Early Data Release (runs 1336, 1339, 1356, and 1359; see EDR). The depth of the SIRTF First Look Survey will range from $m_{\mathrm{AB}} \sim 20$ at $\sim 4 \mu \mathrm{m}$ to $m_{\mathrm{AB}} \sim 15$ at $70 \mu \mathrm{m}$ and to $m_{\mathrm{AB}} \sim 13$ at $160 \mu \mathrm{m}$. There are $\sim 150$ SDSS-FIRST sources in that region, including $\sim 20$ radio-loud quasars. In addition, the SIRTF Wide-Area Infrared Extragalactic (SWIRE) survey will include about $35 \mathrm{deg}^{2}$ of sky that is going to be observed by SDSS ( $\sim 75 \%$ of the overlapping area is already observed by the SDSS imaging survey). The sensitivity of the SWIRE survey will be $2-5$ times better than that of the SIRTF First Look Survey. The final SDSS-SIRTF-FIRST overlapping region will include $\sim 1500$ SDSS-FIRST sources with $\sim 200$ radio-loud quasars; the majority of these sources are expected to be detected by SIRTF. Such a large sample with detailed optical, infrared, and radio data will be of unprecedented size and quality.

We thank Tim McKay, Paul Wiita, and Moshe Elitzur for their careful reading of the manuscript. We are grateful to the referee, Carlos De Breuck, for many insightful suggestions that helped improve the final version. Ž. I., R. H. L., D. S., and G. R. K. acknowledge generous support by Princeton University. M. A. S. acknowledges the support of National Science Foundation grant AST 00-71091. Partial support for this work was provided by the National Aeronautics and Space Administration (to K. M. ) through

\footnotetext{
${ }^{54}$ For details see http://sirtf.caltech.edu/SSC/fls/extragal
}

Chandra Fellowship grant PF9-10006 awarded by the Smithsonian Astrophysical Observatory for NASA under contract NAS 8-39073. P. B. H. acknowledges financial support from Chilean grant FONDECYT/1010981 and a Fundación Andes grant. The FIRST Survey is supported in part under the auspices of the Department of Energy by Lawrence Livermore National Laboratory under contract W7405-ENG-48 and the Institute for Geophysics and Planetary Physics. The Sloan Digital Sky Survey is a joint project of the University of Chicago, Fermilab, the Institute for Advanced Study, the Japan Participation Group, Johns Hopkins University, the Max Planck Institute for Astronomy, the Max Planck Institute for Astrophysics, New Mexico State University, Princeton University, the United States Naval Observatory, and the University of Washington. Apache Point Observatory, site of the SDSS, is operated by the Astrophysical Research Consortium. Funding for the project has been provided by the Alfred P. Sloan Foundation, the SDSS member institutions, NASA, the NSF, the Department of Energy, the Japanese Monbukagakusho, and the Max Planck Society. The SDSS Web site is http://www.sdss.org.

\section{APPENDIX A}

\section{SUMMARY OF DATA SAMPLES USED IN ANALYSIS}

\section{A1. MATCHED SAMPLES}

We matched $2.53 \times 10^{7}$ SDSS sources and 107,654 FIRST sources from $1230 \mathrm{deg}^{2}$ of sky. There are 37,210 matches within $3^{\prime \prime}$ and 29,528 matches within 1".5. Sources matched within 1 1".5 and brighter than $r^{*}=21$ are separated into 15,683 optically resolved sources (galaxies) and 3225 optically unresolved sources (quasars). A subsample from SDSS Early Data Release includes 10,084 matches within $3^{\prime \prime}$, those brighter than $r^{*}=21$ are separated into 1999 quasars and 8085 galaxies.

SDSS spectra are available for a subset of 5454 matched sources from a $774 \mathrm{deg}^{2}$ large region. The spectroscopic matched sample includes 4300 galaxies and 1154 quasars.

\section{A2. CONTROL SAMPLES}

The control imaging sample includes 190,577 unresolved sources (stars and quasars) and 304,147 resolved sources (galaxies) selected from $103 \mathrm{deg}^{2}$ of sky. The control spectroscopic sample includes 141,920 galaxies and 20,085 quasars from a $1030 \mathrm{deg}^{2}$ large region (all available spectra at the time of writing).

\section{APPENDIX B}

\section{A SPECULATION ON THE FRACTION OF HEAVILY OBSCURED QUASARS}

The quasars detected by FIRST but not by SDSS may belong to the population of anomalously optically faint quasars. The most popular argument for the existence of such objects is the expected dust obscuration when the line 
of sight passes through the optically thick torus surrounding the central engine, as advocated by the "unified" models (e.g., Antonucci 1993 and references therein). While there are examples of objects consistent with such an explanation (e.g., Gregg et al. 2002), it is not known how large this population is. It has been suggested that these objects may be as populous as the optically selected quasars (Fall \& Pei 1993; Francis, Whiting, \& Webster 2000).

The data presented here suggest that the distribution of the radio-to-optical flux ratio, $R_{i}$, is independent of redshift and absolute luminosity. Assuming that the observed $R_{i}$ distribution is applicable to all quasars, it can be utilized, together with optical counts of quasars from other surveys deeper than SDSS, to predict the number of quasars detected by FIRST and not detected by SDSS. This prediction can then be compared with the estimated number of FIRST quasars without SDSS counterparts. This method essentially compares the $R_{i}$ distribution determined for SDSS-FIRST quasars with the best guess of what the $R_{i}$ distribution may be for FIRST-only quasars.

The main uncertainty in this method comes from the unknown fraction of quasars in the FIRST-only sample; assuming the same fraction as determined for SDSS-FIRST quasars (17\%), we find that there are 3.3 times as many quasars with $i^{*}>21.5$ and $t<16$ as quasars with $i^{*}<21.5$ and $t<16$. On the other hand, with the extreme assumption that all FIRST-only sources are quasars, the upper limit on this ratio is 17 . If the predicted ratio is much smaller than these estimates, then a population of optically obscured quasars can be invoked to explain the discrepancy. from

We compute the expected number of FIRST-only quasars

$$
N_{\mathrm{RL}}\left(i^{*}>21.5, t<16\right)=\int_{21.5}^{\infty} n\left(i^{*}\right) f_{\mathrm{RL}}\left(i^{*}\right) d i^{*},
$$

where $n\left(i^{*}\right)$ describes the differential optical counts, and $f_{\mathrm{RL}}\left(i^{*}\right)$ is the fraction of all quasars with optical magnitude $i^{*}$ that have $t<16$. The latter is obtained from (valid for $\left.i^{*}>18.5\right)$

$$
f_{\mathrm{RL}}\left(i^{*}\right)=C_{\mathrm{RL}}\left[\frac{\int_{0.4\left(i^{*}-16\right)}^{\infty} \phi\left(R_{i}\right) d R_{i}}{\int_{1}^{\infty} \phi\left(R_{i}\right) d R_{i}}\right] .
$$

All quasars with $t<16$ and $i^{*}>18.5$ are radio-loud $\left(R_{i}>1\right)$, hence the index " RL." Note that the $i *$ integration only formally goes to infinity; the effective range is $i^{*} \lesssim 26$ because most quasars have $R_{i} \lesssim 4$.

The constant $C_{\mathrm{RL}}=0.08$, representing the fraction of quasars with $R_{i}>1$, and the $R_{i}$ distribution, $\phi\left(R_{i}\right)$, are determined at the bright end. The dashed line in the bottom panel in Figure 19 shows a best Gaussian fit to the observed $\phi\left(R_{i}\right)$ distribution; it is centered at $R_{i}=2.8$ with $\sigma=0.8$. Deep optical quasar counts show flattening for $i^{*} \sim 20$ (assuming $i^{*} \sim r^{*}$ ), and follow a $\log (n)=C+0.3 i^{*}$ relation in the range $20 \lesssim i^{*} \lesssim 22.5$ (Pei 1995). Pei's model, which explains well the available observations for $i^{*}<22.5$, predicts that this relation extends to at least $i^{*} \sim 24$. Based on this relation, we investigate two possibilities: (1) this relation is valid for $i^{*}>20$, and (2) it applies to $20<i^{*}<22.5$, and the quasar counts drop to zero for $i^{*}>22.5$. The second assumption is an extreme relation that maximizes the number of quasars that can be attributed to the " obscured" population.
This simple model predicts that the number of quasars with $t<16$ and $i^{*}>21.5$ is 45 times as large as the number of quasars with $t<16$ and $i^{*}<21.5$ for possibility 1 , and 6 times as large for possibility 2 . These values are in the same range as those implied by the counts of FIRST-only sources and demonstrate that there is no compelling need to invoke a significant population of anomalously optically faint quasars. However, we caution that the uncertainty of the predicted counts is large because of the unknown fraction of quasars in the FIRST-only sample and because quasar counts for $21.5<i^{*}<26$ are only weakly constrained. In particular, it cannot be ruled out at a high confidence level that FIRST-only sources include a substantial population of heavily obscured quasars (possibly as large as the population selected by UV excess).

\section{APPENDIX C}

\section{A COMMENT ON THE DEFINITION OF RADIO LOUDNESS}

Two definitions of radio loudness are found in the literature. Schmidt (1970) proposed the use of radio-tooptical flux ratios (or, equivalently, spectral indices; see $\S 2.2$ ) because it appeared that they are distributed independently of optical luminosity and redshift. On the other hand, Peacock, Miller, \& Longair (1986) proposed that the radio luminosity should be used to quantify the radio loudness. The choice of a radio-loudness measure has important physical implications: the radio-to-optical flux ratio is a proper quantity for analysis if the radio and optical emissions are correlated; on the other hand, if the radio emission is independent of optical emission, then the radio luminosity is the quantity of interest. Here we compare both definitions for objects with redshifts from the SDSS spectra and find that they produce similar radio-loud/radio-quiet classifications. This similarity is a consequence of strong selection effects in flux-limited samples.

Figure 37 displays the absolute radio magnitude, $M_{t}$, versus the radio-to-optical flux ratio, $R_{i}$, for the 1154 quasars detected by FIRST. We compute $R_{i}$ using eq. (5) and $M_{t}$ from

$$
M_{t}=-2.5 \log \left(\frac{L_{\text {radio }}}{L_{A B}}\right) \text {, }
$$

where

$$
L_{\text {radio }}=\frac{4 \pi D_{L}^{2}}{(1+z)^{1+\alpha_{r}}} F_{\text {int }}
$$

is the specific radio luminosity, and $L_{\mathrm{AB}}=4.345 \times 10^{13} \mathrm{~W}$ $\mathrm{Hz}^{-1}$ is the specific luminosity of a source whose specific flux is $3631 \mathrm{Jy}$ at a distance of $10 \mathrm{pc}$. We assumed a radio spectral index $\alpha_{r}=-0.5$ and, for consistency with Stern et al. (2000) adopted $H_{0}=50 \mathrm{~km} \mathrm{~s}^{-1} \mathrm{Mpc}^{-1}$ and a $\Omega_{\mathrm{M}}=1, \Omega_{\Lambda}=0$ universe in computing the luminosity distance, $D_{L}$. As is evident, $R_{i}$ and $M_{t}$ are well correlated. Furthermore, the separations between the radio-loud and radio-quiet objects suggested by other workers $\left(R_{i} \sim 1\right.$ and $L_{\text {radio }} \sim 10^{24} h_{50}^{-2} \mathrm{~W}$ $\mathrm{Hz}^{-1}$, corresponding to $\left.M_{t}=-25.9\right)$ appear qualitatively consistent with each other (the two classifications are the same for $88 \%$ of the objects). 


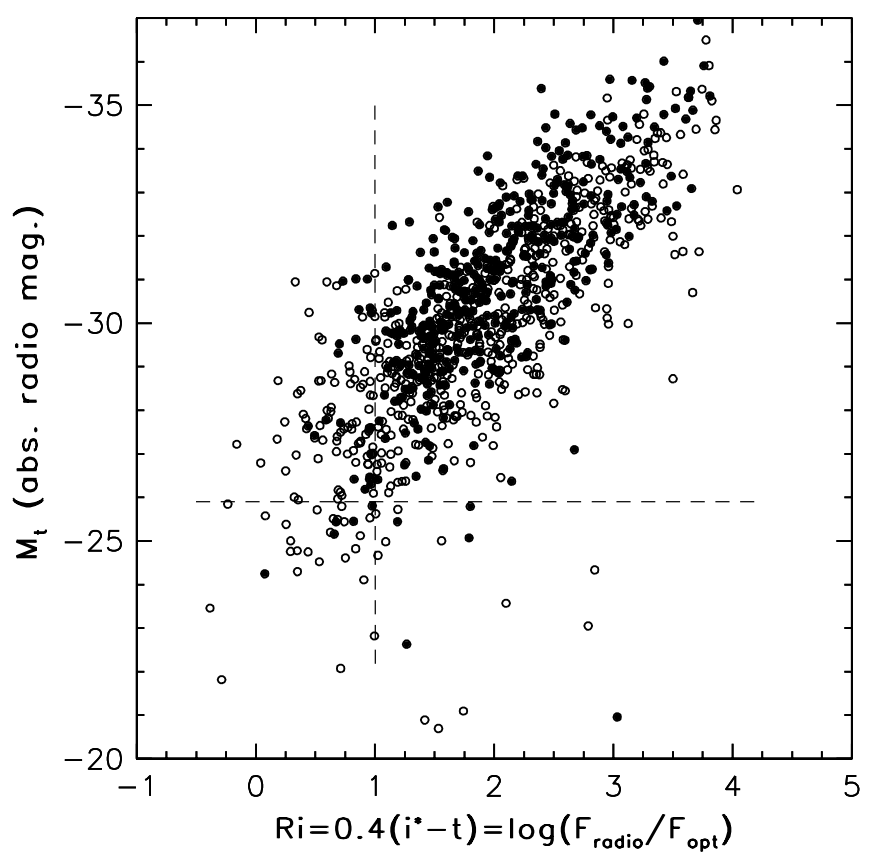

FIG. 37.-Distribution of SDSS-FIRST quasars in the radio-luminosity (absolute magnitude $M_{t}$ ) vs. radio-to-optical flux ratio, $R_{i}$, plane. All 1154 SDSS quasars are marked by open circles. A subsample of 531 objects with $i^{*}<19$ and $t<15.5$ is marked by filled circles. The vertical and horizontal lines are the traditional division lines between the radio-loud and radioquiet quasars. Note that most quasars have the same classification in both $M_{t}$ - and $R_{i}$-based schemes.

It is somewhat surprising that a dimensionless quantity that measures the shape of SED, $R$, is so well correlated with the (dimensional) radio power, $L_{\text {radio }}$. Furthermore, $R$ is practically independent of redshift, while $L_{\text {radio }}$ is a strong function of redshift at constant apparent magnitude. In principle, such a correlation could be a consequence of a physical relationship between optical and radio emission; for example, the luminosity of main-sequence stars is correlated with the shape of the optical SED. However, we find instead that this correlation is due to several selection effects caused by the quasar redshift distribution and very different slopes of the optical and radio number counts $(0.87$ vs. 0.14 , respectively), as follows.

For a sample following a number count relation $\log (n)=C+k m, 90 \%$ of the sample is within $k^{-1} \mathrm{mag}$ from the faint cutoff. Thus $90 \%$ of the SDSS-FIRST sample is within $\sim 1$ mag of the optical faint cutoff and within $\sim 7$ mag of the radio faint cutoff. The minimum value of $R_{i}$ is $0.4\left(i_{\min }-t_{\max }\right)$, where $t_{\max } \sim 16$ is the radio faint limit, and $i_{\min }$ is the optical bright limit. Since $90 \%$ of the sources are within $\sim 1$ mag of the faint optical limit ( $\sim 19)$, only $10 \%$ of the sample can have $R_{i} \lesssim 0.8$, as observed. The maximum value of $R_{i}$ is $0.4\left(i_{\max }-t_{\min }\right)$, where $i_{\max } \sim 19$ is the optical faint limit, and $t_{\min }$ is the radio bright limit. A negligible number of sources are brighter than $t=9$, and thus practically no sources have $R_{i}>4$.

Given $R_{i}$, the variance in $M_{t}$ (the vertical width of the displayed correlation; $\sigma \sim 1.5 \mathrm{mag}$ ) reflects the variances in $i^{*}$ and the distance modulus $\left(M_{t}=i^{*}-D M-2.5 R_{i}\right)$. The latter dominates the scatter in $M_{t}$ because the scatter in $i^{*}$ is small because of the steep optical counts. The scatter in the distance modulus is determined by the distribution of redshifts; the distribution of distance moduli has a median of
$44.5 \mathrm{mag}$ and an equivalent Gaussian width of $1.4 \mathrm{mag}$. The upper limit is a consequence of the upper limit on redshift, and the lower limit is due to the fast increase of the number of quasars per unit redshift interval with redshift. This narrow distribution of distance moduli explains the observed upper (bright) and lower (faint) limits on $M_{t}$ for a given $R_{i}$. The slope of the observed correlation, $d M_{t} / d R_{i}=-2.5$, simply reflects the relationship between $R_{i}$ and $t$. We conclude that the difference in the slopes of the radio and optical number count relations, together with the observed redshift distribution, is responsible for the apparent correlation between $M_{t}$ and $R_{i}$ and there is no intrinsic correlation.

As a result of this selection effect the observed bimodal distribution of $R$ maps into a biased bimodal distribution of $L_{\text {radio }}$. An unbiased distribution of $L_{\text {radio }}$ cannot be determined with the sample discussed here. In particular, a distribution of data points in $L_{\text {radio }}$ versus redshift plane (analogous to Fig. 21) has a strong bias in $R$ that depends on both quantities (only sources with $R_{i}<0.4$ $\left[i^{*}\right.$ cutoff- $\left.t\left(M_{t}, z\right)\right]$ are sampled, where $i^{*}$ cutoff is the optical faint flux limit). For the same reason, the radio luminosity distributions for galaxies ( $\S 5$ ) and quasars from SDSS-FIRST sample cannot be directly compared because of the significantly different redshift ranges over which they are sampled. A proper determination of the radio luminosity function requires identification and redshift determination for all FIRST sources brighter than some radio flux limit, as discussed by, e.g., Willot et al. (1998) and references therein.

\section{APPENDIX D}

\section{THE 6 to 20 CENTIMETER SPECTRAL INDEX FOR QUASARS AND GALAXIES}

The referee pointed out that there is a loophole in our argument based on the Kellerman et al. sample because

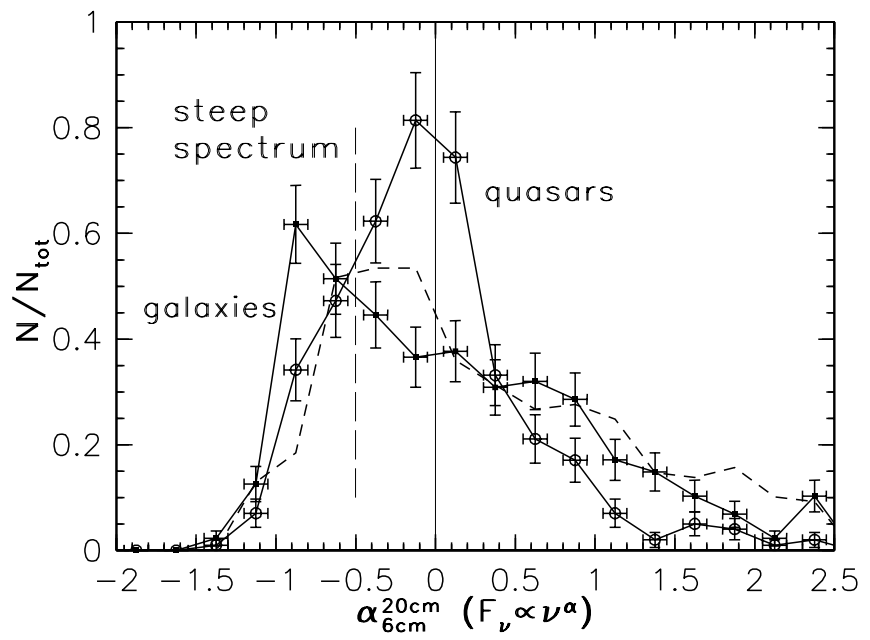

Fig. 38.-Distribution of the 6-20 cm spectral index for quasars (open circles) and galaxies (filled squares) detected by FIRST, SDSS, and GB6 surveys. The vertical long-dashed line at $\alpha_{6}^{20}=-0.5$ shows the boundary between steep-spectrum and flat-spectrum sources. Note that the $\alpha_{6}^{20}$ distribution of FIRST-GB6 sources without an SDSS counterpart (shortdashed line) appears as a linear combination of the quasar and galaxy distributions. 
their observations were obtained at $6 \mathrm{~cm}$ : if the distribution of the 6 to $20 \mathrm{~cm}$ spectral index for quasars has a long tail extending to infinity (i.e., their SED would drop precipitously between 6 and $20 \mathrm{~cm}$ ), then the $R_{i}$ distribution could also have such a tail, rather than a local maximum in the range $-2<R_{i}<0$. In order to determine the distribution of the 6 to $20 \mathrm{~cm}$ spectral index, we have matched SDSSFIRST sources from the EDR area to the Green Bank GB6 survey at $6 \mathrm{~cm}$ (Gregory et al. 1996). The distribution of distances between FIRST and GB6 positions indicates that a good choice for the matching radius is $60^{\prime \prime}$.

The distribution of the 6 to $20 \mathrm{~cm}$ spectral index $\left(F_{\nu} \propto \nu^{\alpha}\right)$,

$$
\alpha_{6}^{20}=0.765(t-s)
$$

where $s$ is the radio $\mathrm{AB}$ magnitude at $6 \mathrm{~cm}$ for 398 quasars observed by SDSS, FIRST, and GB6 is shown in Figure 38 by open circles. Instead of a tail extending to infinity, for the vast majority of quasars $\alpha_{6}^{20}<1.5$. Unless this distribution drastically changes for sources fainter than $1 \mathrm{mJy}$ at $20 \mathrm{~cm}$, the Kellerman et al. results indicate that most of quasars have $R_{i}>-2$, implying a bimodal $R_{i}$ distribution.

For completeness, we also show the $\alpha_{6}^{20}$ distribution for 350 optically resolved SDSS-FIRST-GB6 sources by filled squares. The difference between the two distributions is remarkable. The vertical long-dashed line at $\alpha_{6}^{20}=-0.5$ shows the common separation boundary between steepspectrum and flat-spectrum sources. It is noteworthy that the $\alpha_{6}^{20}$ distribution of FIRST-GB6 sources without an SDSS counterpart, shown by the short-dashed line, appears as a linear combination of the quasar and galaxy distributions. This provides further indication that the quasar-togalaxy number ratio is similar for radio sources with and without SDSS counterparts ( $§ 3.8$ and Figs. 9 and 10).
Antonucci, R. 1993, ARA\&A, 31, 473

Bahcall, N. A., \& Fan, X. 1998, ApJ, 504, 1

Baldwin, J. A., Phillips, M. M., \& Terlevich, R. 1981, PASP, 93, 5

Bartelmann, M., \& White, S. D. M. 2002, A\&A, 388, 732

Becker, R. H., White, R. L., \& Helfand, D. J. 1995, ApJ, 450, 559

Blanton, M., et al. 2001, AJ, 121, 2358

Carlberg, R. G., et al. 2001, ApJ, 563, 736

Chen, B., et al. 2001, ApJ, 553, 184

Colless, B., et al. 2001, MNRAS, 328, 1039

Condon, J. J., O'Dell, S. L., Puschell, J. J., \& Stein, W. A. 1981, ApJ, 246, 624

Condon, J. J., Cotton, W. D., Greisen, E. W., Yin, Q. F., Perley, R. A., Taylor, G. B., \& Broderick, J. J. 1998, AJ, 115, 1693

Dalcanton, J. J. 1996, ApJ, 466, 92

De Breuck, C. 2000, Ph.D. thesis, Univ. Leiden

Eisenstein, D., et al. 2001, AJ, 122, 2267 (E01)

Fall, S. M., \& Pei, Y. C. 1993, ApJ, 402, 479

Fan, X., et al. 2001, AJ, 121, 54

Finlator, K., et al. 2000, AJ, 120, 2615 (F00)

Fomalont, E. B., Kellermann, K. I., Partridge, R. B., Windhorst, R. A., \& Richards, E. A. 2002, preprint (astro-ph/0201441)

Francis, P. J., Whiting, M., \& Webster, R. 1999, Publ. Astron. Soc. Australia, 17, 56

Fukugita, M., Ichikawa, T., Gunn, J. E., Doi, M., Shimasaku, K., \& Schneider, D. P. 1996, AJ, 111, 1748

Gregg, et al. 2002, ApJ, 564, 133

Gregory, P. C., Scott, W. K., Douglas, K., \& Condon, J. J. 1996, ApJS, 103,427

Gunn, J. E., et al. 1998, AJ, 116, 3040

Hooper, E. J., Impey, C. D., Foltz, C. B., \& Hewett, P. C. 1995, ApJ, 445, 62

Ivezić, Ž., et al. 2000, AJ, 120, 963 2002a, in preparation 2002b, in preparation (Paper II)

Kennicutt, R. C. 1992, ApJS, 79, 255

Kellermann, K. I., Sramek, R., Schmidt, M., Shaffer, D. B., \& Green, R. 1989, AJ, 98, 1195

Kewley, L. J., Dopita, M. A., Sutherland, R. S., Heisler, C. A., \& Trevena, J. 2001, ApJ, 556, 121

Kim, R. S. J., et al. 2002, AJ, 123, 20

Knapp, G. R., et al. 2002, in preparation (K02)

Lupton, R. H., Gunn, J. E., \& Szalay, A. S. 1999, AJ, 118, 1406

Lupton, R. H., et al. 2002, in preparation

Madgwick, D. S. et al. 2002, MNRAS, 333, 133

Magliocchetti, M., \& Maddox, S. J. 2002a, MNRAS, 330, 241

Magliocchetti, M., et al. 2002b, MNRAS, 333, 100

\section{REFERENCES}

Magliocchetti, M., et al. 2000, MNRAS, 318, 1047

Magliocchetti, M., Maddox, S. J., Lahav, O., \& Wall, J. V. 1998, MNRAS, 300,257

McMahon, R. G., White, R. L., Helfand, D. J., \& Becker, R. H. 2001, ApJ, in press

Machalski, J., \& Godlowski, W. 2000, A\&A, 360, 463

Menou, K., et al. 2001, ApJ, 561, 645

Miller, L., Peacock, J. A. \& Mead, A. R. G. 1990, MNRAS, 244, 207

Oke, J. B., \& Gunn, J. E. 1983, ApJ, 266, 713

Osterbrock, D. E., \& de Robertis, M. M. 1985, PASP, 97, 1129

Peacock, J. A., Miller, L., \& Longair, M. S. 1986, MNRAS, 218, 265

Pei, Y. 1995, ApJ, 438, 623

Petrosian, V. 2001, preprint (astro-ph/0112467)

Pier, J. R. et al. 2002, AJ, submitted

Richards, G., et al. 2001, AJ, 121, 2308

2002, AJ, 123, 2945

Sadler, E. M., et al. 2002, MNRAS, 329, 227

Schlegel, D., Finkbeiner, D. P., \& Davis, M. 1998, ApJ, 500, 525

Schmidt, M. 1970, ApJ, 162, 371

Schneider, D. P., et al. 2002, AJ, 123, 567

Schneider, D. P., Gunn, J. E., \& Hoessel, J. G. 1983, ApJ, 264, 337

Serjeant, S., et al. 1998, MNRAS, 294, 494

Shimasaku, K., et al. 2001, AJ, 122, 1238

Stern, D., Djorgovski, S. G., Perley, R. A., de Carvalho, R. R., \& Wall, J. V. 2000, AJ, 119, 1526

Stocke, J. T., Morris, S. L., Weymann, R. J., \& Foltz, C. B. 1992, ApJ, 396, 487

Stoughton, C., et al. 2002, AJ, 123, 485 (EDR)

Strateva, I., et al. 2001, AJ, 122, 1861 (S01)

Strauss, M., et al. 2002, AJ, 124, 1810

Strittmatter, P. A., Hill, P., Pauliny-Toth, I. I. K., Steppe, H., \& Witzel, A. 1980, A\&A, 88, L12

Tremonti, C., et al. 2002, in preparation

Urry, C. M., \& Padovani, P. 1995, PASP, 107, 803

Vanden Berk, D. E., et al. 2002, in preparation

Veilleux, S., \& Osterbrock, D. E. 1987, ApJS, 63, 295

Waddington, I., Windhorst, R. A., Dunlop, J. S., Koo, D. C., \& Peacock, J. A. 2000, MNRAS, 317, 801

White, R. L., Becker, R. H., Helfand, D. J., \& Gregg, M. D. 1997, ApJ, 475,479

White, R. L., et al. 2000, ApJS, 126, 133

Willot, C. J., Rawlings, S., Blundell, K. M., \& Lacy, M. 1998, MNRAS, 300,625

Windhorst, R. A., Miley, G. K., Owen, F. N. Kron, R. G., \& Koo, D. C. 1985, ApJ, 289, 494

York, D. G., et al. 2000, AJ, 120, 1579 